\title{
General Truthfulness Characterizations Via Convex Analysis
}

RAFAEL M. FRONGILLO, University of Colorado, Boulder

IAN A. KASH, University of Illinois at Chicago

We present a model of truthful elicitation which generalizes and extends mechanisms, scoring rules, and a number of related settings that do not qualify as one or the other. Our main result is a characterization theorem, yielding characterizations for all of these settings, including a new characterization of scoring rules for non-convex sets of distributions. We generalize this model to eliciting some property of the agent's private information, and provide the first general characterization for this setting. We combine this characterization with duality to give a simple construction to convert between scoring rules and randomized mechanisms. We also show how this characterization gives a new proof of a mechanism design result due to Saks and Yu.

\section{INTRODUCTION}

Information elicitation, the gathering of information from an agent by a principal, is a key problem in economics, statistics, machine learning, and finance. In these settings, one is interested in obtaining the preferences of an agent, a probability distribution from an expert, the desired prediction from an algorithm, and summary statistics of the risk of a portfolio, respectively. They key challenge in all of these settings is to reward the agent in such a way that the agent will truthfully reveal his knowledge rather than being encouraged to reveal some incorrect version. A central question in all these literatures has been to characterize all the ways this can be achieved; that is, to characterize all the truthful mechanisms, proper scoring rules, proper loss functions, or elicitable properties, respectively. Many variants on such characterization theorems have been proved. (See Section 1.2 for a partial list.) Moreover, the proofs of these results all use various tools from convex analysis; in particular, the characterizations tend to be in terms of properties of convex functions and their subgradients.

Despite this commonality of question and technique, the literature on mechanism design has proceeded essentially independent of these other literatures and vice versa. Further, many characterizations are presented for a particular setting, and it is not immediately clear how they would apply to others. As a result, there are many theorems in the literature whose proofs are slight variations on existing results to adapt them to a new setting. While ex post the variations are slight, ex ante the needed changes were frequently not obvious and required significant effort to pin down. An excellent example of this is the scoring rules characterization by Gneiting and Raftery 2007], which has had at least three variants used in various settings Boutilier 2012, Chen and Kash 2011; Cid-Sueiro 2012].

In this paper we address these two problems by formulating a model of information elicitation which is general enough to encompass all these variants, and providing a characterization for it. This obviates the need for additional new theorems in any future application that fits into our framework. Further, it provides a clearer understanding of the connections between mechanism design and scoring rules which allows us to translate results from one domain to the other.

Our model consists of a single agent endowed with some type $t$ known only to the agent, who is asked to reveal his type to the principal. After doing so, the principal gives the agent a score $\mathrm{A}\left(t^{\prime}, t\right)$ that depends on both the agent's reported type $t^{\prime}$ and his true type $t$. We allow $A$ to be quite general, with the main requirement being that $A\left(t^{\prime}, \cdot\right)$ is an affine function (linear transformation plus a constant) of the true type $t$, and seek to understand when it is optimal for the agent to truthfully report his type. Given this truthfulness condition, it is immediately clear why convexity plays a central role-when an agent's type is $t$, we desire the score for telling the truth to satisfy $\mathrm{A}(t, t)=\sup _{t^{\prime}} \mathrm{A}\left(t^{\prime}, t\right)$, which means this 
"consumer surplus" function $G(t):=\mathrm{A}(t, t)$ must be convex as the pointwise supremum of affine functions.

One special case of our model is mechanism design with a single agent, where the designer wishes to select an outcome based on the agent's type. In this setting, $\mathrm{A}\left(t^{\prime}, \cdot\right)$ can be thought of as the allocation and payment given a report of $t^{\prime}$, which combine to determine the utility of the agent as a function of his type. In this context, $\mathrm{A}(t, t)$ is the consumer surplus function (or indirect utility function), and Myerson's well-known characterization [1981] states that, in single-parameter settings, a mechanism is truthful if and only if the consumer surplus function is convex and its derivative (or subgradient at points of non-differentiability) is the allocation rule. More generally, this remains true in higher dimensions (see Rochet 1985]). Note that here the restriction that $\mathrm{A}\left(t^{\prime}, \cdot\right)$ be affine is without loss of generality, because we view types as functions and function application is a linear operation. (See Section 2.2 for more details.)

Another special case is a scoring rule, also called a proper loss in the machine learning literature, where an agent is asked to predict the distribution of a random variable and given a score based on the observed realization of that variable. In this setting, types are distributions over outcomes, and $\mathrm{A}\left(t^{\prime}, t\right)$ is the agent's subjective expected score for a report that the distribution is $t^{\prime}$ when he believes the distribution is $t$. As an expectation, this score is linear in the agent's type. Gneiting and Raftery [2007] unified and generalized existing results in the scoring rules literature by characterizing proper scoring rules in terms of convex functions and their subgradients.

Further, the generality of our model allows it to include settings that do not quite fit into the standard formulations of mechanisms or scoring rules. These include counterfactual scoring rules for decision-making Othman and Sandholm 2010, Chen and Kash 2011. Chen et al. 2011, proper losses for machine learning with partial labels Cid-Sueiro 2012, mechanism design with partial allocations Cai et al. 2013, responsive lotteries Feige and Tennenholtz 2010, and mechanisms for crowdsourcing categorical information Shah and Zhou 2015.

In many settings, it is difficult, or even impossible, to have agents report an entire type $t \in \mathcal{T}$. For example, when allocating a divisible good (e.g. water), a mechanism that needs to know how much an agent would value each possible allocation requires him to submit an infinite-dimensional type. Even type spaces which are exponential in size, such as those that arise in combinatorial auctions, can be problematic from an algorithmic perspective. Moreover, in many situations, the principal is uninterested in all but some small aspect of an agent's private type. For example, the information is often to be used to eventually make a specific decision, and hence only the information directly pertaining to the decision is actually needed - why ask for the agent's entire probability distribution of rainfall tomorrow if a principal wanting to choose between \{umbrella, no umbrella\} would be content with its expected value, or even just whether she should carry an umbrella or not?

It is therefore natural to consider an indirect elicitation model where agents provide some sort of summary information about their type. Such a model has been studied in the scoring rules literature, where one wishes to elicit some statistic, or property, of a distribution, such as the mean or quantile Savage 1971, Osband and Reichelstein 1985, Lambert et al. 2008; Gneiting 2011. We follow this line of research, and extend the affine score framework to accept reports from a different (intuitively, much smaller) space than $\mathcal{T}$.

\subsection{Our Contribution}

Our main theorem (Theorem 2.3) is a general characterization theorem that generalizes and extends known characterization theorems for proper scoring rules (substantially) and truthful mechanisms (slightly). We also survey applications to related settings and show our theorem can be used to provide characterizations for them as well, including new results about mechanism design with partial allocation and responsive lotteries. Thus, our theorem 
eliminates the need to independently derive characterizations for such settings. We further generalize our theorem to the case where the desired report is a function of the private information (a property) rather than the full information (Theorem 4.5) and provide a variant of this characterization which makes use of convex duality (Theorem 5.5). Finally, we conclude by examining cases where the set of possible reports is finite, which Lambert and Shoham 2009 showed correspond to power diagrams, a generalization of Voronoi diagrams (see Section 6). We extend their result to settings where the private information need not be a probability distribution, and give a tight characterization for a particular restricted "simple" case. We also give an explicit construction for generating power diagrams from other measures of distances via a connection to Bregman Voronoi diagrams Boissonnat et al. 2007.

1.1.1. Scoring Rules. Our contribution to the scoring rules literature is a characterization of proper scoring rules for non-convex sets of distributions, the first of its kind. As motivation, note that it is very natural to ask for scoring rules which are proper with respect to a particular family of distributions, e.g. if the principal was convinced that the agent's belief came from such a family, but many common families are non-convex, including most exponential families (Gaussian, Poisson, exponential, Laplace, Pareto, etc.) Nielsen and Garcia 2009. Moreover, general non-convex sets of distributions have proven useful as a way of separating informed and uninformed experts Babaioff et al. 2011; Fang et al. 2010. Despite how natural and useful it is to restrict to the non-convex case, no characterization was known.

We give such a characterization, which surprisingly shows that the only scoring rules which are proper for a non-convex $\mathcal{P}$ are those which can be extended to a proper scoring rule on Conv $\mathcal{P}$; in other words, one does not gain flexibility by ruling out distributions in the "interior" of $\mathcal{P}$. Interestingly, the main technical tool we need to extend the proof to this case comes from the mechanism design literature, where characterizations for non-convex type spaces have been previously established. Additionally, we show that properness of a scoring rule is a local property, in the sense that it suffices to verify it in a neighborhood around each distribution. See Section B.2 and Corollary 2.7.

1.1.2. Property Elicitation. The two subsequent generalizations of our main theorem provide the first general characterizations for arbitrary elicitable properties, which capture only part of the agent's private information (e.g. the mean of a probability distribution rather than the distribution itself). The first is essentially a direct generalization of Theorem 2.3 , which keeps the same general structure but adds the constraint that the convex function must be flat on sets of types which share an optimal report. In addition to serving as our main tool to derive the remainder of our results, this theorem provides several ways to show that a property is not elicitable (by showing that no such convex function can exist). This allows us to show that the smallest confidence interval containing a given amount of mass is not elicitable, thus settling an open question.

The second result is a transformation of this theorem using duality, which shows that there is a strong sense in which properties are subgradients of convex functions. Relatedly, we introduce the notion of direct elicitability, where the the property to be elicited is the subgradient rather than a link function applied to it. We also use this result to introduce notions of dual properties and scores, which gives a new construction to convert between scoring rules and randomized mechanisms (see Construction 1). Subsequent work has made use of these theorems to derive new results on linear and vector-valued properties Frongillo and Kash 2015a, elicitation complexity [Frongillo and Kash 2015b], and peer prediction [Frongillo and Witkowski 2016].

1.1.3. Mechanism Design. For mechanism design, our contributions are more modest. Our characterization is a minor extension of Archer and Kleinberg's characterization, removing a technical assumption Archer and Kleinberg 2008, Theorem 6.1]. We show how many 
previous results about implementability and revenue equivalence can be translated into our framework, but do not introduce significant new results. Instead, the main interest of our approach is that by translating economic questions into convex analysis questions, we can simplify some of the proofs and expose the underlying intuition. Additionally, we show how known results about scoring rules yield a new proof of an implementability theorem due to Saks and $\mathrm{Yu} 2005$.

1.1.4. Novel Elicitation Settings. Perhaps the most useful direct application of our main theorem is to elicitation settings that do not quite match the standard frameworks of scoring rules or mechanism design, as it immediately provides a characterization for such settings. In Section 3 we demonstrate the versatility of our characterization by surveying five recent such examples (on decision rules, proper losses for partial labels, mechanism design with partial allocations, responsive lotteries, and crowdsourcing), and showing how our results could have been applied.

1.1.5. Summary of Novel Results. Since our results cover a range of applications and include many reframings or small extensions of existing results, we summarize the main novel results here.

(1) A general characterization theorem for many non-standard applications;

(2) A characterization of scoring rules for non-convex sets of distributions;

(3) Scoring rules are proper iff they are locally proper;

(4) A new geometric proof of the Saks-Yu 2005 result on implementable mechanisms;

(5) A characterization of elicitability for arbitrary properties;

(6) The smallest confidence interval is not elicitable;

(7) A new construction to convert between scoring rules and randomized mechanisms.

\subsection{Related Work}

The similarities between mechanisms and scoring rules were noted by (among others) Fiat et al. 2013, who gave a construction to convert mechanisms into scoring rules and vice versa, and Feige and Tennenholtz 2010, who gave techniques to convert both to "responsive lotteries." Further, techniques from convex analysis have a long history in the analysis of both models (see Gneiting and Raftery 2007, Vohra 2011). However, we believe that our results use the "right" representation and techniques, which leads to more elegant characterizations and arguments. For example, the construction used by Fiat et al. has the somewhat awkward property that the scoring rule corresponding to a mechanism has one more outcome than the mechanism did, a complication absent from our results. Similarly, the constructions used by Feige and Tennenholtz only handle special cases and they claim "there is no immediate equivalence between lottery rules and scoring rules," while we can give such an equivalence. So while prior work has understood that there is a connection, the nature of that connection has been far from clear.

A large literature in mechanism design has explored characterizations of when allocation rules can be truthfully implemented; see e.g. McAfee and McMillan 1988; Jehiel et al. 1996; Jehiel et al. 1999, Jehiel and Moldovanu 2001; Saks and Yu 2005/, Bikhchandani et al. 2006, \begin{tabular}{|l|l|l|l|l|}
\hline Müller et al. 2007, Archer and Kleinberg 2008 & Ashlagi et al. 2010 & Carroll 2012 . Similarly,
\end{tabular} work on revenue equivalence can be cast in our framework as well Myerson 1981, Krishna and Maenner 2001; Heydenreich et al. 2009, Carbajal and Ely 2012. For scoring rules, our work connects to a literature that has used non-convex sets of probability distributions to separate (usefully) informed exports from uninformed experts Fang et al. 2010, Babaioff et al. 2011.

The study of indirect elicitation in scoring rules can be traced to Savage in 1971, who considered the problem of eliciting expected values of random variables Savage 1971]. Osband and Reichelstein 1985 go on to provide a rigorous version, generalizing to expected 
values of functions of the underlying variable. Since then several similar results have appeared with varying assumptions (e.g. Banerjee et al. 2005, Gneiting 2011; Abernethy and Frongillo 2012 ), all of which have recently been unified in Frongillo and Kash 2015a. More generally, many authors have considered other common statistics as well, such as quantiles, ratios of expectations, and expectiles Osband and Reichelstein 1985, Gneiting and Raftery 2007, Gneiting 2011, Grant and Gneiting 2013.

While these and many other examples of specific statistics have appeared in the literature, it was perhaps Osband [1985] and Lambert, Pennock, and Shoham 2008 who first considered the following general problem: given an outcome space $\mathcal{O}$ and an arbitrary map $\Gamma: \Delta(\mathcal{O}) \rightarrow \mathbb{R}$, under what circumstances can we construct a proper scoring rule to elicit $\Gamma(t)$ ? Moreover, what is the full classification of functions $\Gamma$ which can be elicited in this way?

Several authors have made significant contributions toward answering this general question for the case where $\Gamma$ is real-valued Lambert et al. 2008, Lambert 2018, Gneiting 2011; Gneiting and Katzfuss 2014; Steinwart et al. 2014 and vector-valued Osband 1985: Lambert et al. 2008. Lambert and Shoham 2009 also characterized elicitable properties $\Gamma$ which take on finitely many values, showing a connection to power diagrams from computational geometry.

We focus primarily on properties in the scoring rule context because, while non-directrevelation mechanisms are often studied, the focus is not typically on the report itself but on the outcome of the mechanism. However, properties are somewhat more natural in mechanism design with a finite set of allocations. In particular, mechanisms that elicit a ranking over outcomes rather than a utility for each outcome (common in, e.g., matching contexts) are a form of property elicitation, and our results are related to characterizations due to Carroll 2012. Our results about finite properties also provide a new proof of a theorem due to Saks and $\mathrm{Yu}$ that characterizes when allocation rules that select from a finite set of allocations have payments that make them truthful Saks and Yu 2005.

Subsequent to our work, our properties characterizations have been applied in several contexts. They have been used to provide a new unified characterization of linear properties and several results towards to a characterization of vector-valued properties Frongillo and Kash 2015a, as the basis to move from the question of whether a property is elicitable to how difficult it is to elicit Frongillo and Kash 2015b, and to characterize minimal peer prediction mechanisms Frongillo and Witkowski 2016.

\subsection{Notation}

We define $\overline{\mathbb{R}}=\mathbb{R} \cup\{-\infty, \infty\}$ to be the extended real numbers. Given a set of measures $M$ on a space $X$ with $\sigma$-algebra $\mathcal{B}$, a function $f: X \rightarrow \overline{\mathbb{R}}$ is $M$-quasi-integrable if $\int_{X} f(x) d \mu(x) \in \overline{\mathbb{R}}$ for all $\mu \in M$. Let $\Delta(X)$ be the set of all probability measures on $X$. We denote by $\operatorname{Aff}(X \rightarrow Y)$ and $\operatorname{Lin}(X \rightarrow Y)$ the set of functions from $X \subseteq \mathcal{V}_{1}$ to $Y \subseteq \mathcal{V}_{2}$ which are restrictions of affine and linear functions (respectively) from vector space $\mathcal{V}_{1}$ to vector space $\mathcal{V}_{2}$. We write $\operatorname{Conv}(X)$ to denote the convex hull of a set of vectors $X$, the set of all (finite) convex combinations of elements of $X$. Some useful facts from convex analysis are collected in Appendix $\mathrm{A}$

\section{AFFINE SCORES}

We consider a very general model with an agent who has a given type $t \in \mathcal{T}$ and reports some possibly distinct type $t^{\prime} \in \mathcal{T}$, at which point the agent is rewarded according to some score $\mathrm{A}\left(t^{\prime}, t\right)$ which is affine in the true type $t$. This reward we call an affine score. We wish to characterize all truthful affine scores, which incentivize the agent to report her true type $t$. 
Definition 2.1. Let $\mathcal{T} \subseteq \mathcal{V}$ for some vector space $\mathcal{V}$ over $\mathbb{R}$. A function $\mathrm{A}: \mathcal{T} \times \mathcal{T} \rightarrow \overline{\mathbb{R}}$ is an affine score with score set $\mathcal{A} \doteq\{\mathrm{A}(t, \cdot) \mid t \in \mathcal{T}\} \subseteq \operatorname{Aff}(\mathcal{T} \rightarrow \overline{\mathbb{R}})]^{1}$ We say $\mathrm{A}$ is truthful if for all $t, t^{\prime} \in \mathcal{T}$,

$$
\mathrm{A}\left(t^{\prime}, t\right) \leq \mathrm{A}(t, t)
$$

If this inequality is strict for all $t \neq t^{\prime}$, then $\mathrm{A}$ is strictly truthful.

Our characterization uses convex analysis, a central concept of which is the subgradient of a function, which is a generalization of the gradient yielding a linear approximation that is always below the function.

Definition 2.2. Given some function $G: \mathcal{T} \rightarrow \mathbb{R}$, a function $d \in \operatorname{Lin}(\mathcal{V} \rightarrow \overline{\mathbb{R}})$ is a subgradient to $G$ at $t$ if for all $t^{\prime} \in \mathcal{T}$,

$$
G\left(t^{\prime}\right) \geq G(t)+d\left(t^{\prime}-t\right) .
$$

We denote by $\partial G: \mathcal{T} \rightrightarrows \operatorname{Lin}(\mathcal{V} \rightarrow \overline{\mathbb{R}})$ the multivalued map such that $\partial G_{t}$ is the set of subgradients to $G$ at $t$. ${ }^{2}$ We will occasionally overload the $\partial G$ notation to mean $\partial G=$ $\cup_{t \in \mathcal{T}} \partial G_{t}$. We say a parameterized family of linear functions $\left\{d_{t} \in \operatorname{Lin}(\mathcal{V} \rightarrow \overline{\mathbb{R}})\right\}_{t \in \mathcal{T}}$, for $\mathcal{T}^{\prime} \subseteq \mathcal{T}$ is a selection of subgradients if $d_{t} \in \partial G_{t}$ for all $t \in \mathcal{T}^{\prime}$; we denote this succinctly by $\left\{d_{t}\right\}_{t \in \mathcal{T}^{\prime}} \in \partial G$.

For mechanism design, it is typical to assume that utilities are always real-valued. However, the log scoring rule (one of the most popular scoring rules) has the property that if an agent reports that an event has probability 0 , and then that event does occur, the agent receives a score of $-\infty$. Essentially solely to accommodate this, we allow affine scores and subgradients to take on values from the extended reals. In the next paragraph we provide the relevant definitions, but for most purposes it suffices to ignore these and simply assume that all affine scores are real-valued.

It is standard (cf. Gneiting and Raftery 2007]) to restrict consideration to the "regular" case, where intuitively only things like the log score are permitted to be infinite. In particular, an affine score $\mathrm{A}$ is regular if $\mathrm{A}(t, t) \in \mathbb{R}$ for all $t \in \mathcal{T}$, and $\mathrm{A}\left(t^{\prime}, t\right) \in \mathbb{R} \cup\{-\infty\}$ for $t^{\prime} \neq t$. Similarly, a parameterized family of linear functions (e.g. a family of subgradients) $\left\{d_{t} \in \operatorname{Lin}(\mathcal{V} \rightarrow \overline{\mathbb{R}})\right\}_{t \in \mathcal{T}}$ is $\mathcal{T}$-regular if $d_{t}(t) \in \mathbb{R}$ for all $t \in \mathcal{T}$, and $d_{t^{\prime}}(t) \in \mathbb{R} \cup\{-\infty\}$ for $t^{\prime} \neq t$. Likewise, $\mathcal{T}$-regular affine functions have $\mathcal{T}$-regular linear parts with finite constants (i.e. we exclude the constant functions $\pm \infty$ ). For the remainder of the paper we assume all affine scores and parameterized families of linear or affine functions are $\mathcal{T}$-regular, where $\mathcal{T}$ will be clear from context.

We now state, and prove, our characterization theorem. The proof takes Gneiting and Raftery's 2007 proof for the case of scoring rules on convex domains and extends it to the non-convex case using a variant of a technique Archer and Kleinberg 2008 introduced for mechanisms with non-convex type spaces. This technique is essentially that used in prior work on extensions of convex functions [Peters and Wakker 1987, Yan 2012].

TheOREM 2.3. Let an affine score $\mathrm{A}: \mathcal{T} \times \mathcal{T} \rightarrow \overline{\mathbb{R}}$ with score set $\mathcal{A}$ be given. $\mathrm{A}$ is truthful if and only if there exists some convex $G: \operatorname{Conv}(\mathcal{T}) \rightarrow \overline{\mathbb{R}}$ with $G(\mathcal{T}) \subseteq \mathbb{R}$, and some selection of subgradients $\left\{d_{t}\right\}_{t \in \mathcal{T}} \in \partial G$, such that

$$
\mathrm{A}\left(t^{\prime}, t\right)=G\left(t^{\prime}\right)+d_{t^{\prime}}\left(t-t^{\prime}\right) .
$$

\footnotetext{
${ }^{1}$ To define linear functions to $\overline{\mathbb{R}}$, we adopt the convention $0 \cdot \infty=0 \cdot(-\infty)=0$. Thus, any $\ell \in \operatorname{Lin}(\mathcal{V} \rightarrow \overline{\mathbb{R}})$ can be written as $\ell_{1}+\infty \cdot \ell_{2}$ for some $\ell_{1}, \ell_{2} \in \operatorname{Lin}(\mathcal{V} \rightarrow \mathbb{R})$.

${ }^{2}$ It is important to note a deviation from standard terminology here: typically, a "vertical" subgradient, which can take value $\pm \infty$, is called a subtangent (cf. Gneiting and Raftery 2007); here we use subgradient for both cases.
} 
Proof. It is immediate from the subgradient inequality (2) that the proposed form is in fact truthful, as

$$
\mathrm{A}\left(t^{\prime}, t\right)=G\left(t^{\prime}\right)+d_{t^{\prime}}\left(t-t^{\prime}\right) \leq G(t)=G(t)+d_{t}(t-t)=\mathrm{A}(t, t) .
$$

For the converse, we are given some truthful $\mathrm{A}: \mathcal{T} \times \mathcal{T} \rightarrow \overline{\mathbb{R}}$.

By definition, each $\mathrm{A}(t, \cdot)$ is the restriction of an affine function defined on all of $\mathcal{V}$, and we now argue that $\mathrm{A}(t, \cdot)$ is therefore well-defined on $\operatorname{Conv}(\mathcal{T})$. While in general there may be many different affine functions on $\mathcal{V}$ whose restriction to $\mathcal{T}$ is $\mathrm{A}(t, \cdot)$, given any $\hat{t} \in \operatorname{Conv}(\mathcal{T})$ we may represent $\hat{t}$ as a finite convex combination $\hat{t}=\sum_{i=1}^{m} \alpha_{i} t_{i}$ where $t_{i} \in \mathcal{T}$. Thus by their affineness all of these yield the same values on $\operatorname{Conv}(\mathcal{T})$ as one easily sees that $\mathrm{A}(t, \hat{t})=\sum_{i=1}^{m} \alpha_{i} \mathrm{~A}\left(t, t_{i}\right)$. Thus, $\mathrm{A}(t, \cdot)$ is both determined and well-defined on $\operatorname{Conv}(\mathcal{T})$.

Now we let $G(\hat{t}) \doteq \sup _{t \in \mathcal{T}} \mathrm{A}(t, \hat{t})$, which is convex as the pointwise supremum of convex (in our case affine) functions. Since $\mathrm{A}$ is truthful, we in particular have $G(t)=\mathrm{A}(t, t) \in \mathbb{R}$ for all $t \in \mathcal{T}$ by our regularity assumption. Let $\mathrm{A}_{\ell}(t, \cdot)$ denote the linear part of $\mathrm{A}(t, \cdot)$. Then, also by truthfulness, we have for all $t^{\prime} \in \mathcal{T}$ and $\hat{t} \in \operatorname{Conv}(\mathcal{T})$,

$$
G(\hat{t}) \doteq \sup _{t \in \mathcal{T}} \mathrm{A}(t, \hat{t}) \geq \mathrm{A}\left(t^{\prime}, \hat{t}\right)=\mathrm{A}\left(t^{\prime}, t^{\prime}\right)+\mathrm{A}_{\ell}\left(t^{\prime}, \hat{t}-t^{\prime}\right)=G\left(t^{\prime}\right)+\mathrm{A}_{\ell}\left(t^{\prime}, \hat{t}-t^{\prime}\right) .
$$

Hence, $\mathrm{A}_{\ell}\left(t^{\prime}, \cdot\right)$ satisfies (2) for $G$ at $t^{\prime}$, so $\mathrm{A}$ is of the form (3).

In the remainder of this section, we show how scoring rules, mechanisms, and other related models fit comfortably within our framework.

\subsection{Scoring Rules for Non-Convex $\mathcal{P}$}

In this section, we show that the Gneiting and Raftery characterization is a simple special case of Theorem 2.3. and moreover that we generalize their result to the case where the set of distributions $\mathcal{P}$ may be non-convex. We also give a result about local properness derived using tools from mechanism design in Appendix B.2. To begin, we formally introduce scoring rules and show that they fit into our framework. The goal of a scoring rule is to incentivize an expert who knows a probability distribution to reveal it to a principal who can only observe a single sample from that distribution.

Definition 2.4. Given outcome space $\mathcal{O}$ and set of probability measures $\mathcal{P} \subseteq \Delta(\mathcal{O})$, a scoring rule is a function $\mathrm{S}: \mathcal{P} \times \mathcal{O} \rightarrow \overline{\mathbb{R}}$ such that $\mathrm{S}(p, \cdot)$ is $\mathcal{P}$-quasi-integrable for all $p \in \mathcal{P}$ (see below). We say $\mathrm{S}$ is proper if for all $p, q \in \mathcal{P}$,

$$
\mathbb{E}_{o \sim p}[\mathrm{~S}(q, o)] \leq \mathbb{E}_{o \sim p}[\mathrm{~S}(p, o)] .
$$

If the inequality in $(4)$ is strict for all $q \neq p$, then $\mathrm{S}$ is strictly proper.

To incorporate this into our framework, take the type space $\mathcal{T}=\mathcal{P}$. Thus, we need only construct the correct score set of affine functions available to the scoring rule as payoff functions. Intuitively, these are the functions that describe what payment the expert receives given each outcome, but we have a technical requirement that the expert's expected utility be well defined. Thus, following Gneiting and Raftery, we take $\mathcal{F}$ to be the set of $\mathcal{P}$-quasiintegrable functions $f: \mathcal{O} \rightarrow \overline{\mathbb{R}}$, meaning $\int_{\mathcal{O}} f(o) d p(o) \in \overline{\mathbb{R}}$ for all $p \in \mathcal{P}$, and the score set $\mathcal{A}=\left\{p \mapsto \mathbb{E}_{o \sim p} \mathrm{~S}(q, o) \mid q \in P\right\} \subseteq\left\{p \mapsto \int_{\mathcal{O}} f(o) d p(o) \mid f \in \mathcal{F}\right\}$. Note that in this case $\mathcal{A}$ actually contains linear functions of $p$, which are trivially affine.

We now apply Theorem 2.3, which yields the following generalization of Gneiting and Raftery 2007]. 
COROLlaRY 2.5. For an arbitrary set $\mathcal{P} \subseteq \Delta(\mathcal{O})$ of probability measures, a regular ${ }^{3}$ scoring rule $\mathrm{S}: \mathcal{P} \times \mathcal{O} \rightarrow \overline{\mathbb{R}}$ is proper if and only if there exists a convex function $G$ : $\operatorname{Conv}(\mathcal{P}) \rightarrow \mathbb{R}$ with functions $G_{p} \in \mathcal{F}$ such that

$$
\mathrm{S}(p, o)=G(p)+G_{p}(o)-\int_{\mathcal{O}} G_{p}\left(o^{\prime}\right) d p\left(o^{\prime}\right),
$$

where $G_{p}: q \mapsto \int_{\mathcal{O}} G_{p}\left(o^{\prime}\right) d q\left(o^{\prime}\right)$ is a subgradient of $G$ for all $p \in \mathcal{P}$.

Proof. Truthfulness of the given form follows immediately from Theorem 2.3 and our definition of $\mathcal{A}$. For the converse, let $\mathrm{A}: \mathcal{T} \times \mathcal{T} \rightarrow \overline{\mathbb{R}}$ be a given truthful affine score. From the theorem, $\mathrm{A}(p, \cdot)=G(p)+d_{p}(\cdot-p) \in \mathcal{A}$, so we must have $d_{p} \in \mathcal{F}$; the subgradients are then of the form $G_{p}: q \mapsto \int_{\mathcal{O}} d_{p}(o) d q(o)$ as desired.

Importantly, Corollary 2.5 immediately generalizes the characterization of Gneiting and Raftery 2007] to the case where $\mathcal{P}$ is not convex, which is new to the scoring rules literature. One direction of this extension is obvious (if $\mathbf{S}$ is truthful on the convex hull of a set then it is truthful on that set), but the other is not, and is an important negative result in that it rules out the possibility of new scoring rules arising by restricting the set of distributions (as long as the restriction does not change the convex hull of the set).

In the absence of a characterization, several authors have worked in the non-convex $\mathcal{P}$ case. For example, Babaioff et al. 2011 examine when proper scoring rules can have the additional property that uninformed experts do not wish to make a report (have a negative expected utility), while informed experts do wish to make one. They show that this is possible in some settings where the space of reports is not convex. Our characterization shows that, despite not needing to ensure properness on reports outside $\mathcal{P}$, essentially the only possible scoring rules are still those that are proper on all of $\Delta(\mathcal{O})$. We state the simplest version of such a characterization, for perfectly informed experts, here.

Corollary 2.6. Let a non-convex set $\mathcal{P} \subseteq \Delta(\mathcal{O})$ and $\bar{p} \in \Delta(\mathcal{O})-\mathcal{P}$ be given. A scoring rule $\mathrm{S}$ is proper and guarantees that experts with a belief in $\mathcal{P}$ receive a score of at least $\delta_{A}$ while experts with a belief of $\bar{p}$ receive a score of at most $\delta_{R}$ if and only if $\mathbf{S}$ is of the form (5) with $G(p) \geq \delta_{A} \forall p \in \mathcal{P}$ and $G(\bar{p}) \leq \delta_{R}$.

With a similar goal to Babaioff et al., Fang et al. 2010 find conditions on $\mathcal{P}$ for which every continuous "value function" $G: p \mapsto \mathrm{S}(p, p)$ on $\mathcal{P}$ can be attained by some $\mathrm{S}$ with the motivation of eliciting the expert's information when it is known to come from some family of distributions (which in general will not be a convex set). As such, they provide sufficient conditions on particular non-convex sets, as opposed to our result which provides necessary and sufficient conditions for all non-convex sets. Beyond these specific applications, our characterization is useful for answering practical questions about scoring rules. For example, suppose we assume that people have beliefs about probabilities in increments of 0.01 . Does that change the set of possible scoring rules? No. What happens if they have finer-grained beliefs but we restrict them to such reports? They will end up picking a "nearby" report (see the discussion of convexity in Section 4.2.

In Appendix B.2. we show how local truthfulness conditions, where one verifies that an affine score is truthful by checking that it is truthful in a small neighborhood around every point, from mechanism design generalize to our framework. In particular Corollary B.8 shows that local properness (i.e. properness for distributions in a neighborhood) is equivalent to global properness for scoring rules on convex $\mathcal{P}$, an observation that is also new to the scoring

\footnotetext{
${ }^{3}$ This is the same concept as with affine scores: scores cannot be $\infty$ and only incorrect reports can yield $-\infty$.
} 
rules literature. See Appendix B.2 for the precise meaning of (weak) local properness (i.e. truthfulness).

Corollary 2.7. For a convex set $\mathcal{P} \subseteq \Delta(\mathcal{O})$ of probability measures, a scoring rule $\mathrm{S}: \mathcal{P} \times \mathcal{O} \rightarrow \overline{\mathbb{R}}$ is proper if and only if it is (weakly) locally proper.

\subsection{Mechanism Design}

We now show how to view a mechanism as an affine score. First, we formally introduce mechanisms in the single agent case. (See below for remarks about multiple agents.) Then we show how known characterizations of truthful mechanisms follow easily from our main theorem. This allows us to relax a minor technical assumption from the most general such theorem.

Definition 2.8. Given outcome space $\mathcal{O}$ and a type space $\mathcal{T} \subseteq(\mathcal{O} \rightarrow \mathbb{R})$, consisting of functions mapping outcomes to reals, a (direct) mechanism is a pair $(f, p)$ where $f: \mathcal{T} \rightarrow \mathcal{O}$ is an allocation rule and $p: \mathcal{T} \rightarrow \mathbb{R}$ is a payment. The utility of the agent with type $t$ and report $t^{\prime}$ to the mechanism is $U\left(t^{\prime}, t\right)=t\left(f\left(t^{\prime}\right)\right)-p\left(t^{\prime}\right)$; we say the mechanism $(f, p)$ is truthful if $U\left(t^{\prime}, t\right) \leq U(t, t)$ for all $t, t^{\prime} \in \mathcal{T}$.

Here we suppose that the mechanism can choose an allocation from some set $\mathcal{O}$ of outcomes, and there is a single agent whose type $t \in \mathcal{T}$ is itself the valuation function. That is, the agent's net utility upon allocation $o$ and payment $p$ is $t(o)-p$. Thus, following Archer and Kleinberg 2008, we view the type space $\mathcal{T}$ as lying in the vector space $\mathcal{V}=\mathbb{R}^{\mathcal{O}}$. The advantage of this representation is that while agent valuations in mechanism design can generally be complicated functions, viewed this way they are all linear: for any $v_{1}, v_{2} \in \mathcal{V}$, we have $\left(v_{1}+\alpha v_{2}\right)(o)=v_{1}(o)+\alpha v_{2}(o)$. Thus, we have an affine score $\mathrm{A}\left(t^{\prime}, t\right) \doteq U\left(t^{\prime}, t\right)$, with score set $\mathcal{A}=\left\{U\left(t^{\prime}, \cdot\right) \mid t^{\prime} \in \mathcal{T}\right\} \subset\{t \mapsto t(o)+c \mid o \in \mathcal{O}, c \in \mathbb{R}\}$, so that every combination of outcome and payment a mechanism could choose is potentially an element of $\mathcal{A}$.

As an illustration of our theorem, consider the following characterization, due to Myerson 1981, for a single parameter setting (i.e. when the agent's type can be described by a single real number). The result states that an allocation rule is implementable, meaning there is some payment rule making it truthful, if and only if it is monotone in the agent's type.

Corollary 2.9 (Myerson 1981 ). Let $\mathcal{T}=\mathbb{R}_{+}, \mathcal{O} \subseteq \mathbb{R}$, so that the agent's valuation is $t \cdot o$. Then a mechanism $f, p$ is truthful if and only if

(1) $f$ is monotone non-decreasing in $t$,

(2) $p(t)=t f(t)-\int_{0}^{t} f\left(t^{\prime}\right) d t^{\prime}+p_{0}$.

Proof. By elementary results in convex analysis $f$ is a subgradient of a convex function on $\mathbb{R}$ if and only if it is monotone non-decreasing. By Theorem 2.3 , the mechanism is truthful if and only if $f$ is a subgradient of the particular function $G(t)=U(t, t)=t(f(t))-p(t)$, which is equivalent to (i) and the condition $G(t)=\int_{0}^{t} f\left(t^{\prime}\right) d t^{\prime}+C$.

More generally, applying our theorem gives the following characterization. It is essentially equivalent to that of Archer and Kleinberg [2008] (their Theorem 6.1), although our approach allows the relaxation of a technical assumption they term "outcome compactness" which their version requires when the set of types is non-convex.

Corollary 2.10. A mechanism $f, p$ is truthful if and only if there exists a convex function $G: \operatorname{Conv}(\mathcal{T}) \rightarrow \mathbb{R}$ and some selection of subgradients $\left\{d G_{t}\right\}_{t \in \mathcal{T}}$, such that for all $t \in \mathcal{T}, f(t)=d G_{t}$ and $G(t)=t(f(t))-p(t)$. 
Note that we have written $f(t)=d G_{t}$, but the allocation is an outcome $f(t) \in \mathcal{O}$ while the subgradient is a linear function $d G_{t} \in(\mathcal{O} \rightarrow \mathbb{R}) \rightarrow \mathbb{R}$. Recall the natural isomorphism between outcomes $o$ and functions $t \mapsto t(o)$, which means that we can always represent an outcome as a function sending types to their value for that outcome. In that sense, we mean that $d G_{t}$ is the function $d G_{t}: t^{\prime} \mapsto t^{\prime}(o)$ where $o=f(t)$. Note that the intuition that the subgradient is the allocation function still holds up to this technicality.

While we have thus far dealt with single-agent deterministic mechanisms implemented in dominant strategies, this characterization actually applies significantly more broadly. In a sense, extending our characterizations to multiple agents is trivial: a mechanism is truthful if and only if it is truthful for agent $i$ when fixing the reports of the other agents. Hence, we merely apply our characterization to each single-agent mechanism induced by reports of the other agents. This is sufficient for our present study, but there are certainly reasons to take a more nuanced approach to the multi-agent setting - see Section 7 for further discussion. To extend to randomized mechanisms, one can take $f: \mathcal{T} \rightarrow \Delta(\mathcal{O})$ and define $U\left(t^{\prime}, t\right)=\mathbb{E}_{o \sim f\left(t^{\prime}\right)}[t(o)]-p\left(t^{\prime}\right)$, which is still affine in $t$. We can even extend to nonrisk-neutral agents by taking the outcome space to be $\mathcal{O}^{\prime} \doteq \Delta(\mathcal{O})$. Finally, we can extend to Bayesian agents; in the above discussion of the multi-agent setting, take expectations instead of fixing specific types for the other agents.

Of course, mechanism design asks many questions beyond whether a particular mechanism is truthful, and some of these can be reframed as questions in convex analysis. Implementability focuses on the question of when there exist payments that make a given allocation rule truthful. Figure 1 (a) illustrates known characterizations and how they were proved. As it shows, several of them rely on showing equivalence to a condition from convex analysis known as cyclic monotonicity. Instead, in Appendix B, we reprove them in our more general framework by showing equivalence to the condition of being subgradients of a convex function (see Figure 1 (b)). This has three main benefits. First, by exposing the essential convex analysis question, we are able to greatly simplify the proofs of some of these results. For example, the original proof of Theorem B.4 relies on representing the allocation rule using a graph and arguing about the limit behavior of a process of creating paths in that graph. In contrast, our proof simply requires defining a function and showing it is convex with the correct subgradients by elementary arguments. Second, this reframing reveals that these results actually yield, we believe, new results in convex analysis (in particular, Theorems B.3 B.4 and B.6 and Corollary B.9). Third, this approach shows us how to translate known results from mechanism design into new results about scoring rules, as we saw in Section 2.1. While elements of a subgradient-based approach can be found in a variety of work on characterizing implementability (see, e.g., McAfee and McMillan 1988, Jehiel et al. 1996; Jehiel et al. 1999; Jehiel and Moldovanu 2001; Krishna and Maenner 2001; Milgrom and Segal 2002; Bikhchandani et al. 2006 ), this work has tended to use individual facts applied to particular settings, in contrast to our approach of translating mechanism design questions into convex analysis questions. Nevertheless, as these are essentially reframings of known results that do not directly provide new insights for mechanism design, we defer this material to Appendix B.

Revenue equivalence is the question of when all mechanisms with a given allocation rule charge the same prices (up to a constant). Translating this into convex analysis terms, when is the convex function associated with a given selection of a subgradient unique up to a constant? We ask the more general question, what are all the convex functions consistent with a given selection of a subgradient? The result is a theorem, extending a result due to Kos and Messner 2012, that characterizes the possible payments of every truthful mechanism, even those that do not satisfy revenue equivalence. As their analysis essentially applies the natural convex analysis technique, we again defer this material to Appendix C 


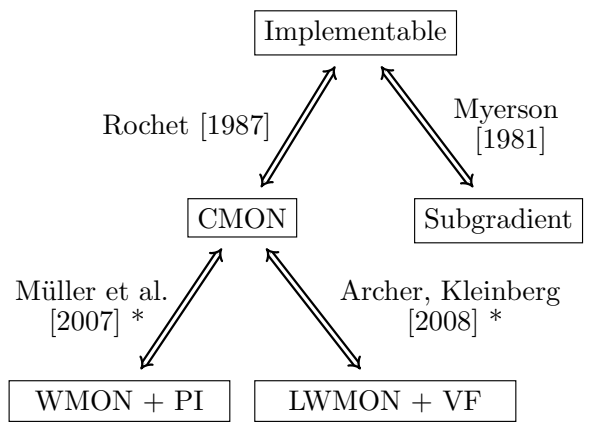

(a)

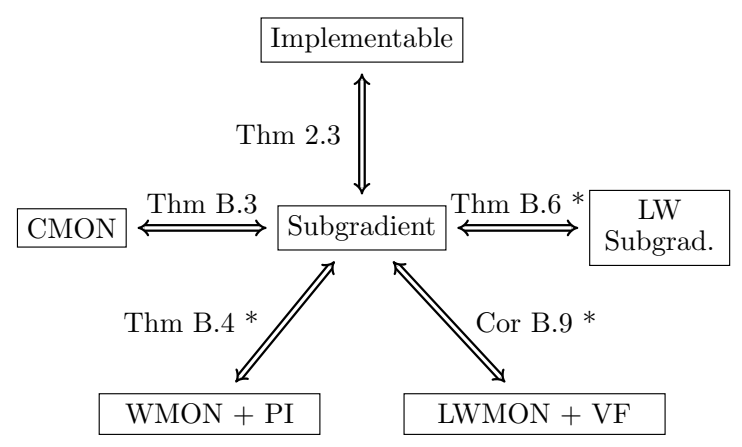

(b)

Fig. 1: Proof structure of existing mechanism design literature (a), and the new proof structure presented in this paper (b). Asterisks $(*)$ denote the requirement that $\mathcal{T}$ be convex. We write CMON for cyclic monotonicity, WMON for weak monotonicity, and PI for path independence. For the other abbreviations, $\mathrm{L}$ is local, $\mathrm{W}$ is weak, and VF is vortex freeness, a condition weaker than path independence introduced in Archer and Kleinberg [2008]. Definitions of these conditions can be found in Appendix B

\section{ADDITIONAL MODELS AS SPECIAL CASES}

A number of other application domains do not quite fit into mechanism design or scoring rules, forcing researchers to adapt results to their particular setting. For example, one may wish to elicit several distributions at once, or partially allocate items or rewards. Fortunately, many such settings can be easily expressed in terms of the more general framework of affine scores. We now briefly survey five such domains, and in each show how our main theorem could have directly provided the characterization ultimately used, rather than requiring effort to conceptualize and prove it.

\subsection{Decision Rules}

A line of work has considered a setting where a decision maker needs to select from a finite set $\mathcal{D}$ of decisions and so desires to elicit the distribution over outcomes conditional on selecting each alternative Othman and Sandholm 2010 Chen and Kash 2011; Chen et al. 2011. Since only one decision will be made and so only one conditional distribution can be sampled, simply applying a standard proper scoring rule does not result in truthful behavior. Applying Theorem 2.3 to this setting characterizes what expected scores must be, from which many of the results in these papers follow.

For example, consider the model of proper scoring rules for decision rules Chen and Kash 2011], which we now describe:

- The decision maker will select an action from $\mathcal{A}=\{1, \ldots, n\}$;

- After $i \in \mathcal{A}$ is chosen, an outcome from $\mathcal{O}=\left\{o_{1}, \ldots o_{m}\right\}$ will be realized, where intuitively the probability of each outcome depends on the action chosen;

— The decision maker asks an expert for the probabilities $P_{i, o}=\operatorname{Pr}[o$ realized $\mid i$ chosen $]$; we will denote by $\mathcal{P}$ the set of all allowable such probability matrices;

- The action is chosen according to a fixed decision rule $D: \mathcal{P} \rightarrow \Delta(\mathcal{A})$ selected in advance by the decision maker, where $D_{i}(P)$ is the probability of choosing action $i$ given the expert reported the matrix $P$;

- The decision maker scores the expert's report based on the chosen action $i$ and materialized outcome $o$, according to the function $S_{i, o}: \mathcal{P} \rightarrow \mathbb{R} \cup\{-\infty\}$. 
Given a belief $P$ and report $Q$, we can therefore write the expert's expected score as $V(Q, P) \doteq \sum_{i, o} D_{i}(Q) P_{i, o} S_{i, o}(Q)$. The definition of (strict) properness for a particular decision rule then follows naturally: a regular scoring rule $\mathrm{S}$ is proper for a decision rule $D$ if $V(P, P) \geq V(Q, P)$ for all $P$ and all $Q \neq P$. It is strictly proper for the decision rule if the inequality is strict.

A key novelty of this setting is the type of the agent: a matrix of conditional probabilities. This is a different object from a distribution over $\mathcal{O}$ or even $\mathcal{O} \times \mathcal{A}$, and thus one cannot use standard scoring rule characterizations. Similarly, the type does not describe the utility of the agent, and hence mechanism design characterizations cannot be applied. For this reason, Chen and Kash 2011] derive a characterization from scratch. Fortunately, the utility of the expert in this setting is an affine score, as the expected score $V(Q, P)$ can be written as a linear combination of the entries of $P$, and therefore is affine in $P$. We can therefore immediately apply our theorem to derive Chen and Kash's 2011 characterization, and even extend it to the case where the set $\mathcal{P}$ of probability matrices is not convex. In the theorem statement we make use of the Frobenius inner product $P: Q \doteq \sum_{i, o} P_{i, o} Q_{i, o}$.

COROLlary 3.1. Given a set of probability matrices $\mathcal{P} \subseteq \Delta(\mathcal{O})^{n}$ A regular scoring rule is (strictly) proper for a decision rule $D$ if and only if

$$
\mathrm{S}_{i, o}(Q)= \begin{cases}G(Q)-G_{Q}: Q+\frac{G_{Q, i, o}}{D_{i}(Q)} & D_{i}(Q)>0 \\ \Pi_{i, o}(Q) & D_{i}(Q)=0\end{cases}
$$

where $G: \operatorname{Conv}(\mathcal{P}) \rightarrow \mathbb{R} \cup\{-\infty\}$ is a (strictly) convex function, $G_{Q}$ is a subgradient of $G$ at the point $Q$ with $G_{Q, i, o}=0$ when $D_{i}(Q)=0$, and $\Pi_{i, o}: \mathcal{P} \rightarrow R \cup\{-\infty\}$ is an arbitrary function that can take a value of $-\infty$ only when $Q_{i, o}=0$.

Proof. By Theorem 2.3, S is (strictly) proper for $D$ if and only if there exists a (strictly) convex $G$ such that $V(Q, P)=G(Q)+d G_{Q}(P-Q)$. That is,

$$
\sum_{i, o} D_{i}(Q) P_{i, o} S_{i, o}(Q)=G(Q)-G_{Q}: Q+\sum_{i, o} G_{Q, i, o} P_{i, o}
$$

or for all $i$ such that $D_{i}(Q) \neq 0$,

$$
\mathrm{S}_{i, o}(Q)=G(Q)-G_{Q}: Q+\frac{G_{Q, i, o}}{D_{i}(Q)} .
$$

When $D_{i}(Q)=0, \mathrm{~S}$ is unconstrained (other than the minimal requirements regarding $-\infty$ for regularity). However, note that our affine score is restricted in that, because $D_{i}(Q)$ is fixed, some choices in $\mathcal{A}$ are not possible to select as subgradients. In particular, it must be that $G_{Q, i, o}=0$ when $D_{i}(Q)=0$.

\subsection{Proper Losses for Partial Labels}

Several variants of proper losses have appeared in the machine learning literature, one of which is the problem of estimating the probability distribution of labels for a new data point when the training data may contain several noisy labels, possibly not even including the correct label. (This is frequently the case, for example, when using crowdsourced labels for training data.) More formally, one wishes to estimate $p \in \Delta_{n}$ where the true label $y \in\{1, \ldots, n\}$ is drawn from $p$. However, instead of observing a sample $y \sim p$ and designing a proper loss $\ell(\hat{p}, y)$, one instead only observes some noisy set of labels $S \subseteq\{1, \ldots, n\}$. Hence, the task is to design a loss $\ell(\hat{p}, S)$ which when minimized over one's data yields accurate estimates of the true $p$.

Recently this problem was studied by Cid-Sueiro 2012 under the assumption that the labels in $S$ are drawn i.i.d. from distribution $q=M p$ for some known $M \in \mathbb{R}^{2^{n} \times n}$, where $p$ is 
the true label distribution. That is, if the actual label is drawn from $p$, the noisy set of labels is drawn from $M p$ (using some indexing of the sets, say lexographical). Cid-Sueiro provides a characterization (his Theorem 4.3) of all proper losses for an even more general version of this setting where $M$ is not known exactly but assumed to be a member of some known class; the loss should be proper for any $M$ in this class. Note that the (negative) payoff $\mathbb{E}_{S \sim M p}[\ell(\hat{p}, S)]=\ell(\hat{p}, \cdot)^{\top} M p$ is linear in the underlying distribution $p$, so our Theorem 2.3 applies and allows us to recover his characterization result. Note that this is essentially a latent observation setting, and the fact that what we observe is a set of labels is in no way necessary; any observed outcome whose distribution has a linear (or affine) relationship with the latent outcome would suffice to apply our theorem.

Rather than introducing the full, general model used by Cid-Sueiro, we show how our theorem applies to yield a characterization for a single, fixed $M$. This is a special case of his Theorem 4.3, generalized to allow restricted sets of probability distributions $\mathcal{P}$.

Corollary 3.2. Let number of labels $n$, matrix $M \in \mathbb{R}^{2^{n} \times n}$, and $\mathcal{P} \subseteq \Delta_{n}$ be given such that $M \mathcal{P}$ has full dimension in the column space of $M$. Let outcome set $\mathcal{O}=2^{\{1, \ldots, n\}}$ be the power set of $\{1, \ldots, n\}$. A regular score $S: \mathcal{P} \times \mathcal{O} \rightarrow \overline{\mathbb{R}}$ is proper if and only if there exists a convex function $G: \operatorname{Conv}(\mathcal{P}) \rightarrow \mathbb{R}$ such that

$$
\ell(\hat{p}, S)=-G(\hat{p})-G_{\hat{p}}^{\top}\left(M^{+} e_{S}\right)+G_{\hat{p}}^{\top} \hat{p},
$$

where $e_{S}$ is a one-hot encoding (indicator vector) of $S, M^{+}$is a left inverse of $M$, and $G_{p}$ is a subgradient of $G$ for all $p \in \mathcal{P}$.

Proof. For the form given, the expected loss is

$$
\begin{aligned}
\mathbb{E}_{S \sim M p}[\ell(\hat{p}, S) & =\mathbb{E}_{S \sim M P}\left[-G(\hat{p})-G_{\hat{p}}^{\top}\left(M^{+} e_{S}\right)+G_{\hat{p}}^{\top} \hat{p}\right] \\
& =-G(\hat{p})-G_{\hat{p}}^{\top} M^{+} M p+G_{\hat{p}}^{\top} \hat{p} \\
& =-G(\hat{p})-G_{\hat{p}} \cdot(p-\hat{p}) .
\end{aligned}
$$

As observed above take $\mathcal{A}=\left\{p \mapsto-\mathbb{E}_{S \sim M p} \ell(q, S) \mid q \in \mathcal{P}\right\}$, each of which is linear in $p$. Propriety of the given form then follows immediately from Theorem 2.3 . For the converse, let $\mathrm{A}: \mathcal{T} \times \mathcal{T} \rightarrow \overline{\mathbb{R}}$ be a given truthful affine score for $\mathcal{T}=\mathcal{P}$. From the theorem, $\mathrm{A}(p, \cdot)=$ $G(p)+d_{p}(\cdot-p) \in \mathcal{A}$. Thus we can write $\ell(\hat{p}, \cdot)^{\top} M p=\mathbb{E}_{S \sim M p}[\ell(\hat{p}, S)]=-G(\hat{p})-G_{\hat{p}} \cdot(p-\hat{p})$. Taking $\hat{\ell}(\hat{p}, \cdot)=\ell(\hat{p}, \cdot)+\left(G(\hat{p})-G_{\hat{p}} \cdot \hat{p}\right) \mathbb{1}$, this means $\hat{\ell}(\hat{p}, \cdot)^{\top} M p=-G_{\hat{p}} \cdot p$ for all $p \in \mathcal{P}$. By our assumption that $M \mathcal{P}$ has full dimension in the column space of $M, \hat{\ell}(\hat{p}, \cdot)^{\top}=-M^{+} G_{\hat{p}}$ for some left inverse $M^{+}$, showing $\ell$ is of the desired form. (If $M \mathcal{P}$ does not have full dimension there may be additional choices of $\ell$ with the correct expected value on $\mathcal{P}$, but the proof otherwise applies.)

\subsection{Mechanism Design with Partial Allocation}

Several mechanism design settings considered in the literature have some form of exogenous randomization, in that "nature" chooses some outcome $\omega$ according to some (often unknown) distribution, and this distribution may depend on the allocation chosen by the mechanism. Examples include sponsored search auctions Feldman and Muthukrishnan 2008, multi-armed bandit mechanisms [Babaioff et al. 2009, and recent work on daily deals [Cai et al. 2013]. The work of Cai, Mahdian, Mehta, and Waggoner 2013 introduces a very general model for such settings, which will be our focus in this subsection.

In the setting of Cai, et al., the mechanism designer wants to elicit two pieces of information: the agent's (expected) value for an item in an auction and the probability distribution of a random variable conditional on that agent winning. Their goal is to understand how the organizer of a daily deal site can take into account the value that will be created for users (as opposed to just the advertiser) when a particular deal is chosen to be advertised. 
(E.g. the site operator may prefer deals that sell to many users over equally profitable deals that sell only to a few because this keeps users interested for future days.) The authors characterize the possible (implementable) ways of quantifying user welfare as a function of the agent's probabilistic belief as to the outcome (of nature) resulting from each allocation. We will show how to recover this characterization as a special case of a more general setting in which a mechanism designer wishes to elicit two pieces of information, but the second need not be restricted to probability distributions.

We begin with a description of the setting of Cai et al. 2013. Let $\mathcal{O}$ be a set of outcomes, and for each outcome $o$ and each agent $i$, let $\Omega^{i, o}$ be some set of events. For example, $o$ could determine which agent wins an auction for the opportunity to advertise a special offer from its business and $\Omega^{i, o}$ could represent the set of numbers of customers that may purchase the deal. Agents each have a valuation function $v^{i}: \mathcal{O} \rightarrow \mathbb{R}$ and a set of beliefs $p^{i, o} \in \Delta\left(\Omega^{i, o}\right)$ for each allocation $o \in \mathcal{O}$, e.g., an expected value for getting to advertise and a probability distribution over the number of customers who accept the deal. The mechanism aggregates all of this information into a single outcome $o$, and additionally choses some payoff function $s^{i}: \Omega^{i, o} \rightarrow \mathbb{R}$, so that the final utility of agent $i$ is $v^{i}(o)+\mathbb{E}_{p^{i, o}}\left[s^{i}\right]$; that is, the winning agent both gets to advertise and accepts a scoring rule contract regarding its prediction of the number of customers. A mechanism is truthful if for all values of $v$ and $p$ for the other agents, agent $i$ maximizes her total utility by reporting $v^{i}$ and $p^{i} \doteq\left(p^{i, o}\right)_{o \in \mathcal{O}}$ truthfully. For additional examples, the standard sponsored search setting has $\Omega^{i, o}=\{$ click, no click $\}$ for $o$ such that $i$ is allocated a slot, and the probabilities $p^{i, o}$ are assumed to be public knowledge. Moreover, the decision rules framework discussed above is a single-agent special case with $v \equiv 0$ and $\Omega^{o}=\Omega^{o^{\prime}}=\Omega$ for all $o \in \mathcal{O}$; of course, unlike the interpretation above, in this setting $o \in \mathcal{O}$ is the allocation/decision while $\Omega$ is the set of outcomes.

Motivated by incorporating the utilities of the end consumers in a daily deal setting, Cai et al. 2013 ask when one can implement an allocation rule of the form $f(v, p)=$ $\operatorname{argmax}_{o \in \mathcal{O}} v(o)+g^{o}\left(p^{o}\right)$, which they interpret as maximizing welfare of the winner plus a term that captures something about the welfare of consumers. In other words, when does there exist some choice of payment making $f$ truthful. The authors conclude that this can be done if and only if $g^{o}$ is convex for each $o \in \mathcal{O}$. In what follows, we will recover this result using our affine score framework.

We first observe that this model can easily be cast as an affine score, as follows. For simplicity, we fix some agent $i$ and focus on the single-agent case; as discussed several times above, this is essentially without loss of generality. The type space is simply (as subset of) the combined private information of the agent,

$$
\mathcal{T} \subseteq\left\{(v, p): v \in \mathcal{O} \rightarrow \mathbb{R}, p \in \prod_{o \in \mathcal{O}} \Delta\left(\Omega^{i, o}\right)\right\} .
$$

By assumption, the utility of the agent upon allocation $o$ and payoff $s$ is simply $v(o)+\mathbb{E}_{p^{o}}[s]$, which is linear in the type $t=(v, p)$ and therefore affine. Thus the net payoff $\mathrm{A}\left(t^{\prime}, t\right)=$ $v\left(o\left(t^{\prime}\right)\right)+\mathbb{E}_{p^{o\left(t^{\prime}\right)}}\left[s\left(t^{\prime}\right)\right]$ is an affine score, with score set $\mathcal{A}=\left\{(v, p) \mapsto v(o)+\mathbb{E}_{p^{o}}[s]: o \in\right.$ $\left.\mathcal{O}, s \in \Omega^{i, o} \rightarrow \mathbb{R}\right\}$, where again we have fixed $i$.

To answer the implementability question of Cai, et al. Cai et al. 2013, we consider a general type space of the form $\mathcal{T} \subseteq \mathcal{V}=\mathcal{V}^{X} \times \mathcal{V}^{Y}$ which can be expressed as a partition into two (subsets of) subspaces. In the daily deal setting, we would have $\mathcal{V}^{X}=\mathbb{R}^{\mathcal{O}}$ and $\mathcal{V}^{Y}=\prod_{o \in \mathcal{O}} \Delta\left(\Omega^{i, o}\right)$, but in general $\mathcal{V}^{Y}$ need not be restricted to probability distributions.

We wish to know when a function $f^{X}: \mathcal{T} \rightarrow \operatorname{Lin}\left(\mathcal{V}^{X} \rightarrow \mathbb{R}\right)$ is extendable, in the sense that there exists some truthful affine score $\mathrm{A}: \mathcal{T} \times \mathcal{T} \rightarrow \overline{\mathbb{R}}$ with score set $\mathcal{A} \subseteq \operatorname{Aff}(\mathcal{V} \rightarrow \mathbb{R})$, and some $f^{Y}: \mathcal{T} \rightarrow \operatorname{Aff}\left(\mathcal{V}^{Y} \rightarrow \mathbb{R}\right)$ such that $\mathrm{A}\left(t^{\prime}, t\right)=f^{X}\left(t^{\prime}\right)\left(t^{X}\right)+f^{Y}\left(t^{\prime}\right)\left(t^{\bar{Y}}\right)$, where of course $t=\left(t^{X}, t^{Y}\right)$. In the daily deals context, $f^{X}$ selects the outcome $o$, formally represented as 
the linear map $v \mapsto v(o)$, and $f^{Y}$ is an expected score of the form $\mathbb{E}_{\omega \sim p^{o}}[s]$, which is affine (here linear) in $p$. Thus, asking whether $f^{X}$ is extendable is equivalent to asking which rules for selecting the outcome $o$ are implementable.

To answer this question, let us introduce some notation. For each $a \in \mathcal{A}$ we write $X(a) \in$ $\operatorname{Lin}\left(\mathcal{V}^{X} \rightarrow \mathbb{R}\right)$ to be the linear part of $a$ on $\mathcal{V}^{X}$, and $Y(a)$ to be the affine part of $a$ on $\mathcal{V}^{Y}$. We will write $X(\mathcal{A}) \doteq\{X(a): a \in \mathcal{A}\}$. In this very general framework, we can show the following.

Theorem 3.3 (Informal). Partial allocation rule $f^{X}$ is extendable if and only if

$$
f^{X}(t) \in \underset{x \in X(\mathcal{A})}{\operatorname{argsup}}\left\{x\left(t^{X}\right)+\sup _{\substack{a \in \mathcal{A} \\ X(a)=x}}\left\{Y(a)\left(t^{Y}\right)\right\}\right\} .
$$

To see this, note that one direction follows from the fact that an affine score is truthful if and only if $\mathrm{A}(t) \in \operatorname{argsup}\{a(t): a \in \mathcal{A}\}$; we simply take the supremum first over $X(\mathcal{A})$ and then over the rest. For the other direction, note that taking $\mathrm{A}\left(t^{\prime}, t\right)=f\left(t^{\prime}\right)\left(t^{X}\right)+y\left(t^{\prime}\right)\left(t^{Y}\right)$ where $y$ is in the argsup of the supremum of eq. (8) gives a truthful affine score.

Returning to the special case of daily deals, let us denote by $a_{o, s} \in \mathcal{A}$ the function $(v, p) \mapsto v(o)+\mathbb{E}_{p^{o}}[s]$. We now see that $f(v, p)$ is implementable if and only if it satisfies

$$
f(v, p) \in \underset{o \in \mathcal{O}}{\operatorname{argsup}}\left\{v(o)+\sup _{s: a_{o, s} \in \mathcal{A}}\left\{\mathbb{E}_{p^{o}}[s]\right\}\right\} .
$$

Thus, letting $g^{o}\left(p^{o}\right)=\sup \left\{\mathbb{E}_{p^{o}}[s]: a_{o, s} \in \mathcal{A}\right\}$, we see that $g^{o}$ is convex as the supremum of affine functions. Moreover, given any collection of convex functions $\left\{g^{o}\right\}_{o \in \mathcal{O}}$, where $g^{o}$ : $\Delta\left(\Omega^{i, o}\right) \rightarrow \mathbb{R}$, we can define $S^{o} \doteq\left\{\omega \mapsto g(p)+d g\left(\mathbb{1}_{\omega}-p\right): p \in \operatorname{dom}(g)\right\}$ and $\mathcal{A} \doteq\left\{a_{o, s}\right.$ : $\left.o \in \mathcal{O}, s \in S^{o}\right\}$, thus recovering each $g^{o}$ in the above expression. It then only remains to show that no other nonconvex function can serve in the argsup; for this one may appeal to the argument of Cai et al. 2013 which observes that the indifference points between different allocations are fixed, thus determining the function in the argsup up to a constant.

A special case of the above, but closer to classical mechanism design, is captured in the following scenario. The mechanism designer has two distinct sets of goods to allocate and wants to design a truthful mechanism that is consistent with a partial allocation rule that determines how the primary goods should be allocated given the agent's preferences over both types of goods. Such mechanisms are characterized by the following informal theorem.

TheOrem 3.4 (Informal). Consider an agent with type $t=\left(t_{1}, t_{2}\right)$. A truthful affine score $\mathrm{A}: \mathcal{T} \times \mathcal{T} \rightarrow \overline{\mathbb{R}}$ with score set $\mathcal{A}$ (which we represent as a set of triples $\left(o_{1}, o_{2}, p\right)$ of first outcome, second outcome, and price) is consistent with a partial allocation rule $f: t \mapsto o_{1}$, if and only if

$$
f(t) \in \underset{o_{1}^{\prime}}{\operatorname{argsup}}\left\{t_{1}\left(o_{1}^{\prime}\right)+\sup _{\substack{\left(o_{1}, o_{2}, p\right) \in \mathcal{A} \\ o_{1}^{\prime}=o_{1}}}\left\{t_{2}\left(o_{2}\right)+p\right\}\right\}
$$

In particular, analogous to the daily deals setting, the mechanism designer is restricted to mechanisms that make decisions based on a convex function of $t_{2}$ (the inner supremum is a pointwise supremum over affine functions and thus convex).

We conclude by noting similarity to recent work of Chambers and Lambert 2014, where a center wishes to elicit an agent's belief about some future event, and to do so multiple times as the event gets closer. The solution proposed is essentially to have the agent choose a menu of scoring functions at time 0 from a menu of menus, and then at time 1 choose 
a score from the menu chosen at time 0. Both the present setting and theirs share this sense of "menu of menus", however one can easily check that the two are incomparable. In particular, the relationship between the final score and the beliefs of the agent can be nonlinear in the Chambers-Lambert model.

\subsection{Responsive Lotteries}

Feige and Tennenholtz [2010] study the problem of how an agent can be incentivized to indirectly reveal his utility function over outcomes by being given a choice of lotteries over those outcomes, an approach with applications to experimental psychology, market research, and multiagent mechanism design. Given a finite set of possible outcomes, the authors give several examples of effective lotteries under the assumption of risk neutrality. In contrast, our approach allows us to give a complete characterization, which highlights the relationship between natural desiderata and underlying geometric properties of the set of possible lotteries, as we now describe.

The authors ask when a responsive lottery is truthful dominant, which is defined as having the following three properties: incentive compatibility, meaning the true utility is among the optimal reports, rational uniqueness, meaning the optimal lottery for a given belief is unique, rational invertibility, meaning every report can be optimal for at most one utility. We would like to relate these notions to simple geometric properties, which we introduce now informally, and formalize later in Definition 3.7. A convex set $K$ is strictly convex if no point on its boundary can be expressed as a convex combination of other points in $K$, and $K$ is smooth if each point on its boundary has a unique unit normal vector. Using the results to follow, we can show that strict truthfulness and continuity of the lottery rule jointly correspond to strict convexity of the lottery set, and uniqueness of the utility given the optimal lottery corresponds to smoothness of the boundary.

COROLlaRY 3.5. A lottery rule $f$ satisfies incentive compatibility and rational uniqueness if and only if $f(x)=\operatorname{argmax}_{p \in K}\langle x, p\rangle$ for $K \subset \Delta_{n}$ compact and strictly convex relative to $\Delta_{n}$. Moreover, $f$ additionally satisfies rational invertibility (and thus is truthful dominant) if and only if $K$ is additionally smooth.

Proof. In this setting, we can see that utility functions are equivalent up to positiveaffine transformations. (Since there are no payments, multiplying the utility of each outcome by a positive constant or adding a constant to the utility for each outcome has no effect on the optimal lottery for an agent.) Thus, we may project the utilities and probability simplex onto the set $V=\left\{x \in \mathbb{R}^{n}: \sum_{i} x_{i}=0\right\}$, which only changes the expected utilities by a constant. We then write these vectors in a basis for $V \cong \mathbb{R}^{n-1}$, normalize the utilities (only scaling them) to the unit sphere in $V$, and apply Theorem 3.8 .

We now turn to the geometric statements needed to establish Corollary 3.5, beginning with some formal definitions. We denote by $\partial K$ the boundary of the set $K \subseteq \mathbb{R}^{n}$.

Definition 3.6. Given a compact convex set $K \subset \mathbb{R}^{n}$, we define the exposed face $F_{K}(t)$ in direction $t \neq 0$ and the normal cone $N_{K}(k)$ at point $k \in \partial K$ by

$$
F_{K}(t)=\underset{k \in K}{\operatorname{argmax}}\langle t, k\rangle, \quad N_{K}(k)=\left\{t \in \mathbb{R}^{n}: k \in F_{K}(t)\right\} .
$$

An exposed face $F_{K}(t)$ is simply the set of points as far in direction $t$ as possible; for example, on a triangle $A B C$ the exposed faces are vertices $A, B, C$ and the edges $\overline{A B}, \overline{B C}, \overline{C A}$. The normal cone $N_{K}(k)$ is simply the set of all valid normal vectors to $K$ at a point $k$ on the boundary of $K$; for the triangle, the normal cone at a point on an edge is simply a ray perpendicular to it, but on a vertex there is a closed cone spanning the exterior angle of the triangle. 
Definition 3.7. We say $K$ is strictly convex if $F_{K}(t)$ is a singleton for all $t \neq 0$. Dually, we say $K$ is smooth if $N_{K}(k)$ is a ray (i.e. $\{\alpha t: \alpha \geq 0\}$ for some $t \neq 0$ ) for all $k \in \partial K$.

To illustrate: a disc is smooth and strictly convex, a triangle is neither, a rounded rectangle is smooth but not strictly convex, and the intersection of two discs is strictly convex but not smooth. We now establish the tight connection between these geometric concepts and the truthfulness conditions in this setting, which will imply Corollary 3.5 .

Theorem 3.8. Let $\mathcal{T}=\left\{t \in \mathbb{R}^{n}:\|t\|_{2}=1\right\}$ be the unit sphere in $\mathbb{R}^{n}$, and let a truthful affine score $\mathrm{A}: \mathcal{T} \times \mathcal{T} \rightarrow \overline{\mathbb{R}}$ with score set $\mathcal{A} \subseteq \operatorname{Lin}\left(\mathbb{R}^{n} \rightarrow \mathbb{R}\right) \cong \mathbb{R}^{n}$ be given. Then $S(t) \doteq \mathrm{A}(t, \cdot)$ is surjective and continuous (as a function to $\mathbb{R}^{n}$ ) and $\mathrm{A}$ is strictly truthful if and only if $\mathcal{A}$ is the boundary of a compact and strictly convex set $K \subset \mathbb{R}^{n}$. $S$ is additionally injective if and only if $K$ is additionally smooth.

Proof. We begin with the first part of the theorem. Let $K$ be compact and strictly convex, and $\mathcal{A}=\partial K$. Then as A is truthful, we must have $S(t) \in \operatorname{argsup}_{a \in \mathcal{A}}\langle t, a\rangle$. As $\mathcal{A}=\partial K$, we may also write $S(t) \in \operatorname{argmax}_{k \in K}\langle t, k\rangle$. Now by strict convexity of $K$, we have for every $a \in \mathcal{A}=\partial K$, there exists some $t \in \mathcal{T}$ such that $\{a\}=\operatorname{argmax}_{k \in K}\langle t, k\rangle$, giving us both surjectivity and strict truthfulness (as $S(t)=a)$. Continuity follows immediately from Berge's Maximum Theorem Ok 2007.

For the converse, let $S$ be strictly truthful, surjective, and continuous. By standard arguments, since $\mathcal{T}$ is a compact subset of $\mathbb{R}^{n}$, we have $\mathcal{A}=S(\mathcal{T})$ is compact as a continuous image of a compact set. Thus, $K \doteq \operatorname{Conv}(\mathcal{A})$ is a compact convex set. Letting $F_{K}(t) \doteq \operatorname{argmax}_{k \in K}\langle t, k\rangle$ be the exposed face of $K$ in direction $t$, we will now show $F_{K}(t)=\{S(t)\}$. First, observe that the extreme points of $K$, ext $(K)$, are a subset of $\mathcal{A}$; otherwise we have $k \in \operatorname{ext}(K) \backslash \mathcal{A}$, so $K \backslash\{k\}$ is a convex set containing $\mathcal{A}$, contradicting the definition of $K=\operatorname{Conv}(\mathcal{A})$. Now we may apply Urruty and Lemaréchal 2001, Proposition A.2.4.6] to express the argmax in terms of the extreme points of $K$, giving us

$$
F_{K}(t) \doteq \underset{k \in K}{\operatorname{argmax}}\langle t, k\rangle=\operatorname{Conv}(\underset{k \in \operatorname{ext}(K)}{\operatorname{argmax}}\langle t, k\rangle) \subseteq \operatorname{Conv}(\underset{a \in \mathcal{A}}{\operatorname{argmax}}\langle t, a\rangle)=\{S(t)\} .
$$

As $K$ is compact, $F_{K}(t)$ is nonempty, and thus $F_{K}(t)=\{S(t)\}$, and additionally we conclude $S(t) \in \operatorname{ext}(K)$. Hence $\mathcal{A}=S(\mathcal{T}) \subseteq \operatorname{ext}(K)$, and as we concluded the reverse conclusion above, we have $\mathcal{A}=\operatorname{ext}(K)$. We now apply Urruty and Lemaréchal 2001, Proposition C.3.1.5] to obtain $\partial K=\bigcup_{t \in \mathcal{T}} F_{K}(t)$, which in turn gives $\partial K=\mathcal{A}$ by surjectivity. Finally, as $\operatorname{ext}(K)=\mathcal{A}=\partial K$, we have strict convexity of $K$.

For the final statement of the theorem, we note that by Urruty and Lemaréchal 2001, Proposition C.3.1.4], we have $k \in F_{K}(t) \Longleftrightarrow t \in N_{K}(k)$. By the above, we already have $F_{K}(t)=\{S(t)\}$ for all $t \in \mathcal{T}$, which implies $N_{K}(k) \cap \mathcal{T}=\{t: S(t)=k\}$. Hence, $N_{K}(a)$ is a ray for all $a \in \mathcal{A}$ if and only if $S$ is injective.

\subsection{Crowdsourcing}

A common technique to provide incentives in crowdsourcing is to ask agents to answer a series of questions, the answers to which a small fraction are known, and then score agents based on how well their responses match the known answers. A classic problem, however, is to design mechanisms which incentivize agents to tell the truth but sufficiently deter "spammers" who may wish to submit low-quality or even useless information in return for small but nonzero rewards. Witkowski et al. 2013 were perhaps the first to address this problem, by incentivizing agents to "pass" on a question if they do not know the answer with sufficient confidence. (In practice, only a small subset of the questions have known answers, and the incentives for these questions are leveraged to obtain worker responses to the other questions. Following standard arguments, if the worker believes this subset to be 
chosen randomly, as can be achieved by a random permutation, then the incentives are the same as if answers were known for every question.)

Similarly, Shah and Zhou 2015 introduce a mechanism which satisfies a simple axiom: if every attempted question is answered incorrectly (among those whose answers are known), the agent should receive 0 payoff. They then show that their mechanism, which is based on thresholds, is the only such mechanism satisfying this axiom. The authors also extend the ideas of Witkowski et al. Witkowski et al. 2013 to allow for multiple "confidence" levels, and define an attempt to be any answer with non-zero confidence.

The proofs in Shah and Zhou 2015] are quite involved, spanning over four pages. Using our framework, we can recover this characterization result in a few paragraphs, as we now demonstrate. The crux of the argument is in translating the axiom to geometric constraints on the mechanism and then applying general results about the geometry of mechanisms with a finite set of allocations. While we apply these results in the proof here, we will discuss them in Section 6 .

To begin, let us introduce the setting of Shah and Zhou [2015] in terms of affine scores. We will take the type of the worker to be a vector $t=\left(p_{1}, \ldots, p_{n}\right) \in[0,1]^{n}$ which encodes the worker's probability of correctly answering each question if it is not skipped, with the outcome being an $n$-dimensional binary vector $o \in\{-1,+1\}^{n}$ encoding for each question whether the agent answered correctly $(+1)$ or not $(-1)$. The "report" of the worker in this formulation would then be to answer or skip, denoted $r \in\{-1,0,+1\}^{n}$ where 0 denotes skipping. Note that in the case of a skip, the outcome is both meaningless and irrelevant. (Of course, in practice a worker would report an actual answer, but the analysis simplifies to the above by abstracting away the separate choices for each answer.) We can think of a payment function $f$ then as taking whether the agent reported and whether the agent would have been correct had she reported and computing a payment based on this; formally, $f:\{0,1\}^{n} \times\{-1,+1\}^{n} \rightarrow \mathbb{R}$. To capture the fact that the designer does not actually know whether the agent was right or wrong when skipping an answer, we require that $f\left(\left(0, r_{-i}\right),\left(-1, o_{-i}\right)\right)=f\left(\left(0, r_{-i}\right),\left(+1, o_{-i}\right)\right)$. Note that while $f$ takes whether the agent reported rather than what the report was, in a slight abuse of notation we use $r$ for both. The expected payment to the worker is $\mathbb{E}_{o_{i} \sim p_{i}} f(r, o)=f(r, \cdot) \cdot t$, which is linear in $t$ and therefore an affine score. Given a confidence threshold parameter $T$, the payment function is deemed incentive-compatible $(I C)$ if for each question $i$, the worker is incentivized to choose $r_{i}=0$ (skip) if $p_{i}<T$, and to choose $r_{i}=1$ (answer the question) if $p_{i}>T$.

We now show how to use our framework to rederive the core results of Shah and Zhou 2015. Let us first restate their no-free-lunch axiom and mechanism in our framework.

AXIOM 1 (No-FREE-LUNCH). If all the answers attempted (not skipped) by the worker are wrong, then the payment is zero.

Define the following multiplicative mechanism given a threshold $T \in(0,1)$ and budget $\mu>0$ which must be fully spent if all questions are answered correctly:

$$
f(r, o)=\mu T^{n-C} \mathbb{1}\{W=0\},
$$

where $C=\left|\left\{i \in\{1, \ldots, n\}: r_{i}=1, o_{i}=1\right\}\right|$ is the number of correct answers, and $W=\left|\left\{i \in\{1, \ldots, n\}: r_{i}=1, o_{i}=-1\right\}\right|=\|r\|_{1}-C$ is the number of incorrect answers (among those not skipped). The name "multiplicative" comes from viewing $f$ as the product of scores for each question: 0 if answered incorrectly, $1 / T$ if answered correctly (recall $T<1$ ), and 1 if skipped.

THEOREM 3.9. The multiplicative mechanism 12 is IC and satisfies the no-free-lunch axiom. 
Proof. The axiom is clearly satisfied by the $\mathbb{1}\{W=0\}$ term. For IC, consider the "property" $\Gamma:[0,1]^{n} \rightarrow\{0,1\}^{n}$ given by $\left.\Gamma(t)=\max _{r \in\{0,1}\right\}^{n} f(r, \cdot) \cdot t$. By definition, the multiplicative mechanism elicits $\Gamma$. Combining Corollary B.8 and the results on finite properties in Section 6, we see that it suffices to check IC at the boundary of each level set of $\Gamma$, corresponding to the types that are indifferent between reporting and skipping for at least one question. This we can check directly, because answering the question $\left(r_{i}=1\right)$ multiplies the payment by $1 / T$ with probability $T$ and makes it 0 with probability $1-T$, a worker is indeed indifferent when $p_{i}=T$ as desired.

Verifying local truthfulness also requires checking types in the the interiors of the level sets but close to the boundary. It follows easily from the indifference of types on the boundary that these types strictly prefer their assigned allocation.

THEOREM 3.10. The multiplicative mechanism is the only IC mechanism satisfying the no-free-lunch axiom.

Proof. Let $f$ be any IC payment function satisfying the no-free-lunch axiom. Again consider the finite property $\Gamma$ from Theorem 3.9 , which by Theorem 6.2 is a power diagram. From the proof of Theorem 6.2 without loss of generality we may take the sites of the corresponding power diagram to be the subgradients of the corresponding "consumer surplus" function given by $G(t)=\max _{r \in\{0,1\}^{n}} f(r, \cdot) \cdot t$, which corresponds to the maximum expected payment a worker can receive given their type $t$.

From the constraint $f\left(\left(0, r_{-i}\right),\left(-1, o_{-i}\right)\right)=f\left(\left(0, r_{-i}\right),\left(+1, o_{-i}\right)\right)$ that the score for skipping does not depend on the true answer, the subgradient for all skipped must be all zeroes.

Now observe that by the structure of the indifference sets where $p_{i}=T$ for exactly one $i$, and the fact that the line segment connecting sites in adjacent cells must be perpendicular to their boundary, we can conclude that the sites must lie on the vertices of an $n$-orthogon (generalization of a rectangle to $n$ dimensions). To see this, consider that for any $r, r^{\prime} \in$ $\{0,1\}^{n}$ differing in entry $i$, the line segment connecting the corresponding sites $s_{r}$ and $s_{r^{\prime}}$ must be perpendicular to the (affine) hyperplane $\left\{t: t_{i}=T\right\}$. Piecing these constraints together shows that fixing the site for $r^{0}=(0, \ldots, 0)$ and each $r^{i}$, which is zero in every entry but $r_{i}=1$, uniquely determines the corresponding $n$-orthogon on which the sites reside as vertices. Moreover, each of these sites is non-zero only in the dimensions corresponding to the questions answered. By the no-free-lunch axiom, all of the weights of the power diagram are 0. (Note that this also shows the no-free-lunch axiom could be (apparently) further weakened to only apply when a single question is answered.) The requirement to pay the budget $\mu$ if all questions are answered correctly then fixes the scaling.

\section{PROPERTY ELICITATION}

We wish to generalize the notion of truthful elicitation from eliciting private information from some set $\mathcal{T}$ to accept reports from a space $\mathcal{R}$ which is different from $\mathcal{T}$. To even discuss truthfulness in this setting, we need a notion of a truthful report $r$ for a given type $t$. We encapsulate this notion by a general multivalued map which specifies all (and only) the correct values for $t$.

Definition 4.1. Let $\mathcal{T}$ be a given type space, where $\mathcal{T} \subseteq \mathcal{V}$ for some vector space $\mathcal{V}$ over $\mathbb{R}$, and $\mathcal{R}$ be some given report space. A property is a multivalued map $\Gamma: \mathcal{T} \rightrightarrows \mathcal{R}$ which associates a nonempty set of correct report values to each type. We let $\Gamma_{r} \doteq\{t \in \mathcal{T} \mid r \in$ $\Gamma(t)\}$ denote the set of types $t$ corresponding to report value $r$.

One can think of $\Gamma_{r}$ as the "level set" of $\Gamma$ corresponding to value $r$. This concept will be especially useful when we consider finite-valued properties in Section 6 . A natural constraint to impose on these level sets is that they be non-redundant, meaning no property value $r$ has a level set entirely contained in another. 
Definition 4.2. Property $\Gamma: \mathcal{T} \rightrightarrows \mathcal{R}$ is redundant if there exist $r, r^{\prime} \in \mathcal{R}$ such that $\Gamma_{r^{\prime}} \subseteq \Gamma_{r}$. Otherwise, $\Gamma$ is non-redundant.

The non-redundancy condition is essentially a bookkeeping tool. If one adds report elements $r^{\prime}$ which are (weakly) dominated by another report $r$, then any time $r^{\prime}$ would be correct, an agent could safely report $r$ instead. Hence, one could think of imposing this condition then as simply "pre-processing" $\Gamma$ to remove any dominated reports.

We extend the notion of an affine score to this setting, where the report space is $\mathcal{R}$ instead of $\mathcal{T}$ itself. Note that the score set $\mathcal{A}=\{\mathrm{A}(r, \cdot) \mid r \in \mathcal{R}\}$ is still a subset of $\operatorname{Aff}(\mathcal{V} \rightarrow \overline{\mathbb{R}})$.

Definition 4.3. An affine score $\mathrm{A}: \mathcal{R} \times \mathcal{T} \rightarrow \overline{\mathbb{R}}$ elicits a property $\Gamma: \mathcal{T} \rightrightarrows \mathcal{R}$ if for all $t$,

$$
\Gamma(t)=\underset{r \in \mathcal{R}}{\operatorname{argsup}} \mathrm{A}(r, t) .
$$

If we merely have $\Gamma(t) \subseteq \operatorname{argsup}_{r \in \mathcal{R}} \mathrm{A}(r, t)$, we say A weakly elicits $\Gamma$. A property $\Gamma: \mathcal{T} \rightrightarrows \mathcal{R}$ is elicitable if there exists some affine score $\mathrm{A}: \mathcal{R} \times \mathcal{T} \rightarrow \overline{\mathbb{R}}$ eliciting $\Gamma$.

Note that it is certainly possible to write down A such the argsup in $(13)$ is not well defined. This corresponds to some types not having an optimal report, which we view as violating a minimal requirement for a sensible affine score. Thus, as we have defined properties to be nonempty, in order for A to be an affine score, we require (13) to be nonempty for all $t \in \mathcal{T}$.

As before, we allow $\mathrm{A}(r, t)$ to take on values in the extended reals to capture scoring rules such as the $\log$ score, so we need a notion of regularity - an affine score $A$ is $\Gamma$-regular if $\mathrm{A}(r, t)<\infty$ always and $\mathrm{A}(r, t) \in \mathbb{R}$ whenever $r \in \Gamma(t)$. We define $\Gamma$-regular linear and affine families similarly 4

\subsection{A First Characterization}

The simplest way to come up with an elicitable property is to induce one from an affine score. For any $\mathrm{A}: \mathcal{R} \times \mathcal{T} \rightarrow \overline{\mathbb{R}}$ with score set $\mathcal{A} \subset \operatorname{Aff}(\mathcal{V} \rightarrow \overline{\mathbb{R}})$, the property

$$
\Gamma^{\mathrm{A}}: t \rightarrow \underset{r \in \mathcal{R}}{\operatorname{argsup}} \mathrm{A}(r, t)
$$

is trivially elicited by $A$ if this argsup is well defined.

Observe also that any affine score $A$ eliciting $\Gamma$ gives rise to a truthful affine score in the original sense - in fact, this is a version of the revelation principle from mechanism design. For each $t$ let $r_{t} \in \Gamma(t)$ be a report choice for $t$; then the affine score $\mathrm{A}^{\mathcal{T}}\left(t^{\prime}, t\right) \doteq \mathrm{A}\left(r_{t^{\prime}}, t\right)$ is truthful. Moreover, by our choices of $\left\{r_{t}\right\}$, we have

$$
G(t) \doteq \sup _{t^{\prime} \in \mathcal{T}} \mathrm{A}^{\mathcal{T}}\left(t^{\prime}, t\right)=\sup _{r \in \mathcal{R}} \mathrm{A}(r, t)
$$

Of course, in general, $A^{\mathcal{T}}$ will not be strictly truthful, since by definition, any reports $t^{\prime}, t^{\prime \prime}$ with $r_{t^{\prime}}=r_{t^{\prime \prime}}$ will have $\mathrm{A}^{\mathcal{T}}\left(t^{\prime}, \cdot\right) \equiv \mathrm{A}^{\mathcal{T}}\left(t^{\prime \prime}, \cdot\right)$. Thus we may think of a property as refining the notion of strictness for a truthful affine score. The connection we draw in Theorem 4.5 is that, in light of (15), a property $\Gamma$ therefore specifies the portions of the domain of $\mathcal{T}$ where $G$ must be "flat". To get at the connection between properties and "flatness", we start with a technical lemma which shows that having the same subgradient at two different points is equivalent to $G$ being flat in between.

Lemma 4.4. Let $G: \operatorname{Conv}(\mathcal{T}) \rightarrow \overline{\mathbb{R}}$ be convex with $G(\mathcal{T}) \subseteq \mathbb{R}$, and let $d \in \partial G_{t}$ for some $t \in \mathcal{T}$. Then for all $t^{\prime} \in \mathcal{T}$,

$$
d \in \partial G_{t^{\prime}} \Longleftrightarrow G(t)-G\left(t^{\prime}\right)=d\left(t-t^{\prime}\right) .
$$

\footnotetext{
${ }^{4}$ The family $\left\{\ell_{r} \in \operatorname{Lin}(\mathcal{V} \rightarrow \overline{\mathbb{R}})\right\}_{r \in \mathcal{R}}$ is $\Gamma$-regular if $\ell_{r}(t) \in \mathbb{R}$ for all $t \in \Gamma_{r}$, and $\ell_{r}\left(t^{\prime}\right) \in \mathbb{R} \cup\{-\infty\}$ for $t^{\prime} \in \mathcal{T} \backslash \Gamma_{r}$. Likewise for $\Gamma$-regular affine functions.
} 
Proof. First, the forward direction. Applying the subgradient inequality (2) at $t^{\prime}$ for $d G_{t}=d$ and at $t$ for $d G_{t^{\prime}}=d$, we have

$$
\begin{aligned}
G\left(t^{\prime}\right) & \geq G(t)+d\left(t^{\prime}-t\right) \\
G(t) & \geq G\left(t^{\prime}\right)+d\left(t-t^{\prime}\right),
\end{aligned}
$$

from which the result follows (as $G(t)$ and $G\left(t^{\prime}\right)$ are finite).

For the converse, assume $G(t)=G\left(t^{\prime}\right)+d\left(t-t^{\prime}\right)$ and let $t^{\prime \prime} \in \mathcal{T}$ be arbitrary. As $G(t)$ and $G\left(t^{\prime}\right)$ are finite, $d\left(t-t^{\prime}\right)$ is as well. Then using the subgradient inequality (2),

$$
G\left(t^{\prime}\right)+d\left(t^{\prime \prime}-t^{\prime}\right)=G\left(t^{\prime}\right)+d\left(t^{\prime \prime}-t\right)+d\left(t-t^{\prime}\right)=G(t)+d\left(t^{\prime \prime}-t\right) \leq G\left(t^{\prime \prime}\right) .
$$

We are now ready to state our first characterization, which in essence says that eliciting a property $\Gamma$ is equivalent to eliciting subgradients of a convex function $G$. Intuitively, by truthfulness the linear part of $\mathrm{A}(r, \cdot)$ must be a subgradient of $G$ at all $t \in \Gamma_{r}$. The lemma shows that this equivalent to flatness, which means we can calculate $G$ on $\Gamma_{r}$ set by picking any type $t_{r} \in \Gamma_{r}$ and following the subgradient. Since all choices of $t_{r}$ lead to the same value, we could just as easily ask for this subgradient $\varphi(r)$ to be reported directly. As subgradients are functions (in this case from $\mathcal{T}$ to $\overline{\mathbb{R}}$ ), we use the curried notation $\varphi(r)(t)$ for the application of this function. Recall that we overload the $\partial G$ notation to refer to the full set of possible subgradients $\partial G=\cup_{t \in \mathcal{T}} \partial G_{t}$.

THEOREM 4.5. Let non-redundant property $\Gamma: \mathcal{T} \rightrightarrows \mathcal{R}$ and $\Gamma$-regular affine score $\mathrm{A}$ : $\mathcal{R} \times \mathcal{T} \rightarrow \overline{\mathbb{R}}$ be given. Then $\mathrm{A}$ elicits $\Gamma$ if and only if there exists some convex $G: \operatorname{Conv}(\mathcal{T}) \rightarrow$ $\overline{\mathbb{R}}$ with $G(\mathcal{T}) \subseteq \mathbb{R}$, some $\mathcal{D} \subseteq \partial G$, some bijection $\varphi: \mathcal{R} \rightarrow \mathcal{D}$ with $\Gamma(t)=\varphi^{-1}\left(\mathcal{D} \cap \partial G_{t}\right)$, and some $\left\{t_{r}\right\}_{r \in \mathcal{R}} \subseteq \mathcal{T}$ satisfying $r^{\prime} \in \Gamma\left(t_{r^{\prime}}\right)$ for all $r^{\prime}$, such that for all $r \in \mathcal{R}$ and $t \in \mathcal{T}$,

$$
\mathrm{A}(r, t)=G\left(t_{r}\right)+\varphi(r)\left(t-t_{r}\right) .
$$

Moreover, if A elicits $\Gamma$ it can be written in this form for any $\left\{t_{r}\right\}_{r \in \mathcal{R}} \subseteq \mathcal{T}$ satisfying $r^{\prime} \in \Gamma\left(t_{r^{\prime}}\right)$ for all $r^{\prime}$.

Proof. For the converse, let $A$ be given of the form (16). We show that it elicits $\Gamma$, i.e. $\Gamma(t)=\operatorname{argsup}_{r \in \mathcal{R}} \mathrm{A}(r, t)$. The third line of the derivation applies Lemma 4.4 .

$$
\begin{aligned}
r \in \Gamma(t) & \Longleftrightarrow r \in \varphi^{-1}\left(\mathcal{D} \cap \partial G_{t}\right) \\
& \Longleftrightarrow \varphi(r) \in \mathcal{D} \cap \partial G_{t} \\
& \Longleftrightarrow \mathrm{A}(r, t)=G(t) \\
& \Longleftrightarrow r \in \underset{r^{\prime} \in \mathcal{R}}{\operatorname{argsup}} \mathrm{A}\left(r^{\prime}, t\right) .
\end{aligned}
$$

Note that any choice of $\left\{t_{r^{\prime}}\right\}_{r^{\prime} \in \mathcal{R}}$ such that $r^{\prime} \in \Gamma\left(t_{r^{\prime}}\right)$ for all $r^{\prime}$ would suffice for Lemma 4.4 .

For the forward direction, assume that affine score $\mathrm{A}$ elicits $\Gamma$. For each $r$, we may extend $\mathrm{A}(r, \cdot)$ to all $\hat{t} \in \operatorname{Conv}(\mathcal{T})$ by linearity as in the proof of Theorem 2.3 , whence we may define $G(\hat{t}) \doteq \sup _{r \in \mathcal{R}} \mathrm{A}(r, \hat{t})$, which is finite for $\hat{t} \in \mathcal{T}$ as $\mathrm{A}$ is $\Gamma$-regular. We wish to show that the choice $\varphi: r \mapsto \mathrm{A}_{\ell}(r, \cdot)$ suffices, where $\mathrm{A}_{\ell}$ denotes the linear part of $\mathrm{A}$, with $\mathcal{D}$ the range of $\varphi$ and $\left\{t_{r}\right\}$ arbitrary satisfying the theorem. Given this construction, we need to check each of the following.

1. $G$ is convex with subgradients $\varphi(\Gamma(t)) \subseteq \partial G_{t}$. Let $t$ and $r \in \Gamma(t)$ be given. We show that $\varphi(r)$ satisfies the property of a subgradient at $t$, and thus $G$ is convex with appropriate 
subgradients.

$$
\begin{aligned}
G(t)+\varphi(r)\left(t^{\prime}-t\right) & =\sup _{r^{\prime} \in \mathcal{R}} \mathrm{A}\left(r^{\prime}, t\right)+\mathrm{A}_{\ell}\left(r, t^{\prime}-t\right) \\
& =\mathrm{A}(r, t)+\mathrm{A}_{\ell}\left(r, t^{\prime}-t\right)=\mathrm{A}\left(r, t^{\prime}\right) \\
& \leq \sup _{r^{\prime} \in \mathcal{R}} \mathrm{A}\left(r^{\prime}, t^{\prime}\right)=G\left(t^{\prime}\right)
\end{aligned}
$$

2. A satisfies eq. (16). This follows from (17) with $t=t_{r}$, as $r \in \Gamma\left(t_{r}\right)$.

3. $\varphi$ is a bijection. By definition, $\mathcal{D}$ is the range of $\varphi$, so we only need to check that it is injective. Suppose for contradiction that $\varphi(r)=\varphi\left(r^{\prime}\right)$. Then, by definition, $\mathrm{A}_{\ell}(r, \cdot)=$ $\mathrm{A}_{\ell}\left(r^{\prime}, \cdot\right)$. Since A elicits $\Gamma$, we have $\mathrm{A}(r, \cdot)=\mathrm{A}\left(r^{\prime}, \cdot\right)$. But then $r \in \Gamma(t) \Longleftrightarrow r^{\prime} \in \Gamma(t)$, contradicting $\Gamma$ being non-redundant.

4. $\Gamma(t)=\varphi^{-1}\left(\mathcal{D} \cap \partial G_{t}\right)$. We already know that $\varphi(\Gamma(t)) \subseteq \partial G_{t}$, so since $\mathcal{D}$ is the range of $\varphi$ we have $\varphi(\Gamma(t)) \subseteq \mathcal{D} \cap \partial G_{t}$. For the other direction, $d \in \mathcal{D} \cap \partial G_{t}$ is $\varphi(r)$ for some $r$. Then by Lemma 4.4. $\mathrm{A}(r, t)=G\left(t_{r}\right)+\varphi(r)\left(t-t_{r}\right)=G(t)$, so $r \in \Gamma(t)$.

As a corollary, we also obtain a better understanding of weak elicitation, which we will need in the following sections.

COROLlaRY 4.6. Let non-redundant property $\Gamma: \mathcal{T} \rightrightarrows \mathcal{R}$ and $\Gamma$-regular affine score $\mathrm{A}: \mathcal{R} \times \mathcal{T} \rightarrow \overline{\mathbb{R}}$ be given. Then A weakly elicits $\Gamma$ if and only if A satisfies (16) with the weaker condition that $\Gamma(t) \subseteq \varphi^{-1}\left(\mathcal{D} \cap \partial G_{t}\right)$.

Proof. We will use the equivalent condition $\varphi(\Gamma(t)) \subseteq \mathcal{D} \cap \partial G_{t}$. Also, recall the definition of $\Gamma^{A}$ in $(14)$, and note that for any affine score $A$, we have $A$ weakly elicits $\Gamma$ if and only if $\Gamma(t) \subseteq \Gamma^{\mathrm{A}}(t)$ for all $t$; that is, if every report in $\Gamma(t)$ maximizes $\mathrm{A}(\cdot, t)$ (but there could be others).

$(\Rightarrow)$ Let $A$ weakly elicit $\Gamma$, which by the above means $\Gamma \subseteq \Gamma^{\mathrm{A}}$. Applying Theorem 4.5 for $\Gamma^{\mathrm{A}}$ we have some $G, \varphi, t_{r}$ such that we can write $\mathrm{A}(r, t)=G\left(t_{r}\right)+\varphi(r)\left(t-t_{r}\right)$ for $\varphi\left(\Gamma^{\mathrm{A}}(t)\right)=\mathcal{D} \cap \partial G_{t}$ and $r \in \Gamma^{\mathrm{A}}\left(t_{r}\right)$. This immediately gives $\varphi(\Gamma(t)) \subseteq \varphi\left(\Gamma^{\mathrm{A}}(t)\right)=\mathcal{D} \cap \partial G_{t}$. It remains to show that $r \in \Gamma\left(t_{r}\right)$, which we will show holds without loss of generality: if $r \notin \Gamma\left(t_{r}\right)$, we at least have $\varphi(r) \in \partial G_{t_{r}}$ by the above, and we have some $\hat{t}_{r}$ such that $r \in \Gamma\left(\hat{t}_{r}\right)$ and $\varphi(r) \in \partial G_{\hat{t}_{r}}$. Now by Lemma 4.4 we see that $G\left(t_{r}\right)-G\left(\hat{t}_{r}\right)=\varphi(r)\left(t_{r}-\hat{t}_{r}\right)$, and thus we may replace $t_{r}$ by $\hat{t}_{r}$ without changing $A$.

$(\Leftarrow)$ Let $\mathrm{A}$ be of the form $(16)$ for $r \in \Gamma\left(t_{r}\right)$ and $\varphi(\Gamma(t)) \subseteq \mathcal{D} \cap \partial G_{t}$ for all $t, r$. As A elicits $\Gamma^{\mathrm{A}}$, we have $\varphi\left(\Gamma^{\mathrm{A}}(t)\right)=\mathcal{D} \cap \partial G_{t}$ and as $\varphi$ is a bijection, $\Gamma(t) \subseteq \Gamma^{\mathrm{A}}(t)$ for all $t$.

Using Corollary 4.6, we see that an affine score A is truthful if and only if it weakly elicits $\Gamma: t \mapsto\{t\}$. Hence, Theorem 4.5 and Corollary 4.6 are actually generalizations of Theorem 2.3 . Of course, we also obtain the following corollary characterizing non-redundant properties.

Corollary 4.7. Non-redundant $\Gamma: \mathcal{T} \rightrightarrows \mathcal{R}$ is elicitable if and only if exists there some convex $G: \operatorname{Conv}(\mathcal{T}) \rightarrow \overline{\mathbb{R}}$ with $G(\mathcal{T}) \subseteq \mathbb{R}$, some $\mathcal{D} \subseteq \partial G$, and some invertible $\varphi: \mathcal{R} \rightarrow \mathcal{D}$ such that $\Gamma(t)=\varphi^{-1}\left(\mathcal{D} \cap \partial G_{t}\right)$.

An important question which would give stronger characterizations is the following:

Question 1. Given non-redundant elicitable $\Gamma$, what are all pairs $G, \mathcal{D}$ such that there exists some bijection $\varphi$ satisfying Theorem 4.5? Equivalently (up to redundancy), given a convex function $G$ with subgradient level sets $L S_{G}(d)=\left\{t: d \in \partial G_{t}\right\}$, what are all the convex functions $G^{\prime}$ with $L S_{G^{\prime}} \equiv L S_{G}$ ? 
In Section 6 we will see that the answer to this question has a lot of structure in the case where $\mathcal{R}$ is finite. In the general case, certainly performing a homothet of the subgradients of $G$ (i.e. scaling $G$ and adding a linear term), will preserve the elicitation structure. However, surely more can be done - the property $\Gamma(t)=\{t\}$ can be elicited with both $G(t)=t^{2} / 2$ and $G(t)=|t|+t^{2} / 2$, which is not a homothet transformation.

While we do not have a complete answer to Question 1 our characterization sheds new light on the structure of elicitable properties in two directions. First, in the scoring rules literature, it is common to assume strong conditions on $\Gamma$ and $\mathcal{R}$, such as $\Gamma$ being a function rather than a multivalued map, and $\Gamma$ being linear [Abernethy and Frongillo 2012] or realvalued [Lambert et al. 2008] to achieve characterizations. In contrast, Theorem 4.5 allows for an extremely general $\Gamma$ and $\mathcal{R}$ and shows us how to construct affine scores for such properties. Second, we can identify features that all elicitable properties share, which provides a means to prove that specific properties are not elicitable.

\subsection{What Properties Are Not Elicitable?}

In the remainder of this section, we examine three features that subgradient mappings of convex functions possess and thus that the level sets of elicitable properties must possess.

Convexity. A well-known property of subgradient mappings is that their level sets are convex (for completeness, we provide a proof in Appendix A).

In light of our characterizations, this fact about convex functions immediately applies to elicitable properties.

Proposition 4.8. For convex functions $G$, the set $\partial G^{-1}(d) \doteq\left\{x \in \operatorname{dom}(G): d \in \partial G_{x}\right\}$ is convex.

Corollary 4.9. If $\Gamma: \mathcal{T} \rightrightarrows \mathcal{R}$ is elicitable, then $\Gamma_{r}$ is convex for all $r$.

To see the corollary, just note that $\varphi(r) \in \partial G_{t} \cap \partial G_{t^{\prime}}$ implies that $\varphi(r) \in \partial G_{\hat{t}}$ for all $\hat{t}=\alpha t+(1-\alpha) t^{\prime}$. Corollary 4.9 was previously known for special cases Lambert et al. 2008; Lambert and Shoham 2009], where it was used to show variance, skewness, and kurtosis are not elicitable, and was also known in mechanism design (i.e. the set of types for which a given (allocation, payment) pair is optimal is convex).

Cardinality. Combining Theorem 4.5 with the fact that finite-dimensional convex functions are differentiable almost everywhere (cf. Aliprantis and Border 2007, Thm 7.26]) yields the following corollary, which shows that elicitable properties have unique values almost everywhere.

COROLlary 4.10. Let $\Gamma: \mathcal{T} \rightrightarrows \mathcal{R}$ be an elicitable property with $\mathcal{T} \subseteq \mathcal{V}=\mathbb{R}^{n}$. If $\mathcal{T}$ is of positive measure in $\operatorname{Conv}(\mathcal{T})$, and $\Gamma$ is non-redundant, then $|\Gamma(t)|=1$ almost everywhere.

In some cases, this statement holds in infinite-dimensional vector spaces as well, provided one uses an appropriate notion of "almost everywhere" (cf. Borwein and Vanderwerff 2010, p. 195] and Aliprantis and Border 2007, p. 274]). One can use this fact to show that $\Gamma(p)=\left\{(a, b): \int_{a}^{b} p(x) d x=0.9\right\}$, the set of $90 \%$ confidence intervals for a distribution $p$, is not an elicitable property. This was previously only known for the case where $p$ has finite support Lambert et al. 2008.

Topology. Finally, we can use tools from topology to draw other conclusions. Combining Theorem 4.5 with a closure property of convex functions [Rockafellar 1997, Thm 24.4] yields the following.

Corollary 4.11. Let $\Gamma: \mathcal{T} \rightrightarrows \mathcal{R}$ be an elicitable property with $\mathcal{T} \subseteq \mathcal{V}=\mathbb{R}^{n}$ convex that can be elicited by a closed, convex $G$. Then $\Gamma_{r}$ is closed for all $r$. 
Requiring $G$ to be closed is a generally mild technical assumption regarding the boundary of of $\mathcal{T}$, and is irrelevant for level sets in the relative interior. While Lambert and Shoham 2009 showed this for finite report spaces $\mathcal{R}$, this more general statement shows, for example, that if $\mathcal{T}=\mathbb{R}$ the property $\Gamma(t)=$ floor $(t)=\max \{z \in \mathbb{Z} \mid z \leq t\}$ is not elicitable. More generally, this often provides a tool for showing that we cannot get around issues of cardinality by finding a tie-breaking rule to make the value unique.

Closure appears is a more delicate attribute to work with in infinite dimensions, but intuitive violations of it can still be used to show that properties are not elicitable. As an illustration, we provide a direct proof that a property is not elicitable. We already saw that confidence intervals are not elicitable due to their cardinality, and therefore a natural way to break these ties, which is also of practical interest, would be to ask for the smallest such interval. Can we elicit this smallest interval, or even just its length? The following sketch shows we cannot. For probability distribution $F$ represented by a CDF, let $\Gamma(F)=\inf \{b-a \mid F(b)-F(a)=1 / 2\}$ be the length of the smallest interval of probability $1 / 2$. Consider the family of distributions with densities $f_{c}$ defined as follows: $f_{c}(x)=1 / 2-c$ for $0 \leq x \leq 1, f_{c}(x)=c$ for $1<x \leq 2$, and $f_{c}(x)=1 / 4$ for $4 \leq x \leq 6$, with corresponding CDFs $F_{c}$. Note that for $0<c<1 / 2, \Gamma\left(F_{c}\right)=\{2\}$ but $\Gamma\left(F_{0}\right)=\{1\}$. Suppose we could elicit $\Gamma$ with scoring rule $\mathrm{S}$. Let $X(F)=\mathrm{S}(2, F)-\mathrm{S}(1, F)$. By elicitability $X\left(F_{c}\right)>0$ for $0<c<1 / 2$ but $X\left(F_{0}\right)<0$, which violates the continuity of $X$ given by linearity of expectation. This argument can be extended to arbitrary properties as follows.

COROLlaRY 4.12. Let $\Gamma: \mathcal{T} \rightrightarrows \mathcal{R}$ be an elicitable property for $\mathcal{T}$ convex, and let $t, t^{\prime} \in \mathcal{T}$ and $t_{\alpha}=\alpha t+(1-\alpha) t^{\prime}$ for $\alpha \in[0,1]$. If $\Gamma\left(t_{\alpha}\right)=\{r\}$ for $\alpha \in[0,1)$, then $r \in \Gamma(t)$.

Proof. Suppose A elicits $\Gamma$, and assume for a contradiction that $r \in \Gamma\left(t_{\alpha}\right)$ for $\alpha \in[0,1)$, but $r \notin \Gamma(t)$. Let $r^{\prime} \in \Gamma(t)$, noting $r^{\prime} \neq r$, and define $X(\alpha) \doteq \mathrm{A}\left(r, t_{\alpha}\right)-\mathrm{A}\left(r^{\prime}, t_{\alpha}\right)$. By affineness of $\mathrm{A}$, we can write $X(\alpha)=\alpha\left(\mathrm{A}(r, t)-\mathrm{A}\left(r^{\prime}, t\right)\right)+(1-\alpha)\left(\mathrm{A}\left(r, t^{\prime}\right)-\mathrm{A}\left(r^{\prime}, t^{\prime}\right)\right)$, which is continuous with respect to $\alpha$. But now $X(\alpha)>0$ for $\alpha<1$ as $r^{\prime} \notin\{r\}=\Gamma\left(t_{\alpha}\right)$, while $X(1)<0$ as $r \notin \Gamma(t) \supseteq\left\{r^{\prime}\right\}$, contradicting continuity of $X$.

Using Corollary 4.12 , we can extend the argument above to show that neither the smallest confidence interval containing mass $\alpha$, defined as $\Gamma_{\alpha}(F)=\operatorname{argmin}_{(a, b)}\{b-a \mid F(b)-F(a)=$ $\alpha$, nor its length, is elicitable with respect to families of distributions which include all piecewise linear CDFs with a finite number of pieces.

\section{DUALITY IN PROPERTY ELICITATION}

In the previous section, we saw from Theorem 4.5 that in a strong sense an elicitable property $\Gamma$ is like a subgradient mapping of a convex function. In order to explore notions of duality, we first remove the word "like" from the previous sentence - we look at properties which are subgradient mappings. This exercise has two main benefits. First, it gives us a concrete tool to reason about properties, by working directly with a convex function rather than through some map $\varphi$. Second, it gives a new framework to discuss duality in elicitation, as has been observed between scoring rules and prediction markets Hanson 2003, Chen and Vaughan 2010; Abernethy and Frongillo 2012, Chen et al. 2013, Abernethy et al. 2013.

\subsection{Subgradient Elicitation}

Now that we have formalized the relationship between the report space and subgradients of convex functions, we can see what the "canonical" properties look like: those which are (subsets of) subgradient mappings of a convex function. For these properties, we can talk about subgradient elicitation, which roughly speaking means removing the intermediary $\varphi$ between $\mathcal{R}$ and $\partial G$. In fact, for such properties, it makes sense to talk about a convex function itself eliciting $\Gamma$. 
Definition 5.1. A property $\Gamma: \mathcal{T} \rightrightarrows \mathcal{D}$, where $\mathcal{T} \subseteq \mathcal{V}$ and $\mathcal{D} \subseteq \mathcal{V}^{*} \doteq \operatorname{Lin}(\mathcal{V} \rightarrow \overline{\mathbb{R}})$, is subgradient-elicitable if there exists $G: \operatorname{Conv}(\mathcal{T}) \rightarrow \overline{\mathbb{R}}$ convex with $G(\mathcal{T}) \subseteq \mathbb{R}$ such that $\Gamma(t) \subseteq \partial G_{t}$. In this case we say $G$ subgradient-elicits, or just elicits, $\Gamma$.

In other words, $G$ elicits $\Gamma: \mathcal{T} \rightrightarrows \mathcal{D}$ if the $\varphi$ in Theorem 4.5 and Corollary 4.7 is the identity. It is easy to construct an affine score eliciting such a property.

Proposition 5.2. Subgradient-elicitable properties are elicitable.

Proof. Let $\Gamma: \mathcal{T} \rightrightarrows \mathcal{R}$ and $G: \operatorname{Conv}(\mathcal{T}) \rightarrow \overline{\mathbb{R}}$ convex with $G(\mathcal{T}) \subseteq \mathbb{R}$ be given such that $\Gamma(t) \subseteq \partial G_{t}$. Then taking $\mathcal{D}=\mathcal{R}$ and $\varphi=\operatorname{id}_{\mathcal{D}}$, we have by Corollary 4.7 that $\Gamma$ is elicitable. Note that despite $\Gamma(t) \subseteq \partial G_{t}$ we have $\Gamma(t)=\operatorname{argsup}_{r \in \mathcal{R}} \mathrm{A}(r, t)$ because the range of $\Gamma$ is $\mathcal{R}$.

Note that this subgradient elicitability in no way necessary for elicitability, since the report space can be arbitrary. For example, one can take $\Gamma(t) \doteq-\partial G_{t}$ for some $G$, which in general will not be subgradient-elicitable, but still elicitable with $\varphi(r)=-r$ and $G$.

We can also clarify what we mean when we say subgradient elicitation is canonical: every elicitable property gives rise to a subgradient-elicitable property.

Proposition 5.3. Let $\Gamma$ be an elicitable property, elicited by $\mathrm{A}(r, t)=G\left(t_{r}\right)+\varphi(r)(t-$ $\left.t_{r}\right)$. Then $\Gamma^{\varphi}(t)=\varphi(\Gamma(t))$ is subgradient-elicitable.

Proof. Simply keep $G$ and take $\operatorname{id}_{\mathcal{D}}$ as the new $\varphi$.

In other words, properties are subsets of subderivative mappings, up to some bijection, or link function, taking them to some other report space $\mathcal{R}^{\prime}$; see also the discussion following Theorem 5.5 .

As a final remark, we note a few observations about subgradient elicitation. One first notices that the $G$ eliciting some $\Gamma$ is not unique, as $G^{\prime} \doteq G+c$ will also elicit $\Gamma$ for any constant $c$. But these are the only convex functions subgradient-eliciting $\Gamma$. Moreover, recovering such a $G$ from $\Gamma$ is easy: simply integrate (a selection of) $\Gamma$ to obtain $G$. Testing whether $\Gamma$ is subgradient-elicitable is less straight-forward, but there are a variety of monotonicity conditions addressing this issue as well (cf Appendix B).

\subsection{Report Duality}

We are now ready to introduce the first of two notions of duality in property elicitation, where we change the report from the type to the dual type, but leave the type itself unchanged. For now, we will take our dual vector space to be all linear functions from $\mathcal{V}$ to $\mathbb{R}$ (not $\overline{\mathbb{R}}$ as above) 5 We begin with the fundamental operation of convex duality, the convex conjugate.

Definition 5.4. Let $\mathcal{V}^{*} \doteq \operatorname{Lin}(\mathcal{V} \rightarrow \mathbb{R})$. The convex conjugate of $G: \mathcal{V} \rightarrow \overline{\mathbb{R}}$, denoted $G^{*}: \mathcal{V}^{*} \rightarrow \overline{\mathbb{R}}$, is given by

$$
G^{*}(d)=\sup _{v \in \mathcal{V}} d(v)-G(v) .
$$

The power of the conjugate is apparent from Fact 4 in Appendix A, which says that $v$ is an optimum of (18), meaning $G^{*}(d)=d(v)-G(v)$, if and only if $d$ is a subgradient at $v$. In this way, the convex conjugate in some sense encodes the subgradients of $G$. Fact 4 lets us further simplify Theorem 4.5, as follows. Note however that we are making an additional assumption, that $G>-\infty$.

\footnotetext{
${ }^{5}$ When the dual space can take on infinite values, the conjugate is not always well-defined, as values of the form $\infty-\infty$ are encountered.
} 
THEOREM 5.5. Let non-redundant property $\Gamma: \mathcal{T} \rightrightarrows \mathcal{R}$ and $\Gamma$-regular affine score $\mathrm{A}$ : $\mathcal{R} \times \mathcal{T} \rightarrow \overline{\mathbb{R}}$ be given with score set $\mathcal{A} \subseteq \operatorname{Aff}(\mathcal{T} \rightarrow \mathbb{R})$. Then $\mathrm{A}$ elicits $\Gamma$ if and only if there exists some convex $G: \operatorname{Conv}(\mathcal{T}) \rightarrow \mathbb{R}$, and bijective $\varphi: \mathcal{R} \rightarrow \mathcal{D}$ with $\mathcal{D} \subseteq \partial G$ satisfying $\varphi(\Gamma(t)) \subseteq \partial G_{t}$, such that for all $r \in \mathcal{R}$ and $t \in \mathcal{T}$

$$
\mathrm{A}(r, t)=\varphi(r)(t)-G^{*}(\varphi(r))
$$

Theorem 5.5 has natural interpretations for both mechanisms and scoring rules. For mechanisms, it captures a version of the taxation principle, that a mechanism can be viewed as a menu of possible allocations $\varphi(r)$ with corresponding prices $G^{*}(\varphi(r))$. It is worth noting however that this posted-price mechanism is not always identical to the original mechanism. Specifically, while the equilibrium payoffs for the posted-price mechanism $\mathrm{A}(r, t)$ are the same as those of the direct revelation mechanism $\mathrm{A}\left(t^{\prime}, t\right)$, the off-equilibrium payoffs need not be equivalent, as the posted-price mechanism may allow reports $\varphi(r) \in \partial G_{t}$ which are not $d G_{t^{\prime}}$ for any $t^{\prime}$. In other words, because the primal-report (i.e., direct) mechanism must choose a single subgradient $d G_{t}$ for every point, if $\left\{d G_{t}\right\}_{\mathcal{T}} \subsetneq \partial G=\mathcal{D}$, the dual-report mechanism can be strictly more expressive.

For scoring rules, Theorem 5.5 captures the relationship between a scoring rule and a prediction market. The prediction market model we refer to is the standard cost-function framework Abernethy et al. 2013, which we briefly describe. A centralized market maker chooses a convex cost function $C$, and traders who wish to buy a bundle of securities $q \in \mathbb{R}^{\mathcal{O}}$ (where the trader will receive $\$ q_{o}$ upon outcome $o$ ) pays $C\left(q^{0}+q\right)-C\left(q^{0}\right)$, where $q^{0}$ is the vector of total purchases made so far in the market. Abstracting away $q^{0}$, we set $G^{*}(q)=C\left(q^{0}+q\right)-C\left(q^{0}\right)$, yielding affine score $\mathrm{A}\left(q^{\prime}, p\right)=\left\langle p, q^{\prime}\right\rangle-G^{*}\left(q^{\prime}\right)$. Hence, scoring rules have the primal report space, and prediction markets the dual, where an optimal share bundle is essentially a subgradient of the scoring rule at the trader's belief. As with posted-price mechanisms, the prediction market can be strictly more expressive.

\subsection{Type Duality and the Duality Quadrangle}

We have seen the first notion of duality, where the report switches from the type space to the dual space. In Appendix D we introduce the second notion, that of dual properties, where we swap the roles of types and reports. Taken together, these two duality notions give four combinations of dual report and type, which form a "duality quadrangle", depicted in Table I. We focus here on the particular instantiation of type space $\Delta(\mathcal{O})$ and dual type space $(\mathcal{O} \rightarrow \mathbb{R})$, based on the classic duality between integrable functions and probability measures; Appendix D generalizes to any dual pair of vector spaces.

As we discussed in Section 5.2, the columns of Table $\mathrm{I}$ are well-understood already. The first gives prediction market duality, the well-known fact that market scoring rules are dual to prediction markets, in the sense of report duality. The second gives the taxation principle, which says that without loss of generality one could think of a mechanism as assigning prices to probability distributions over outcomes $o$. The rows of this table, however, are new, as we swap the nature of the private information itself. In the scoring rule or prediction market setting, an agent has a private distribution (their belief) and the principal gives the agent a utility vector (the score or the bundle of securities), which assigns the agent a real-valued payoff for each possible state of the world. Dually, in a mechanism, the agent possesses a private type encoding their utility for each state of the world, and the principal assigns a distribution over these states.

Thus, in essence, scoring rules are dual mechanisms. In the remainder of this section, we elaborate on this particular connection, in the finite-outcome setting for clarity, giving a concrete construction to convert scoring rules to mechanisms and vice versa. Note that unlike previous constructions (e.g., Fiat et al. 2013]) we can establish a direct bijection between the two objects, and do not require any normalization. 


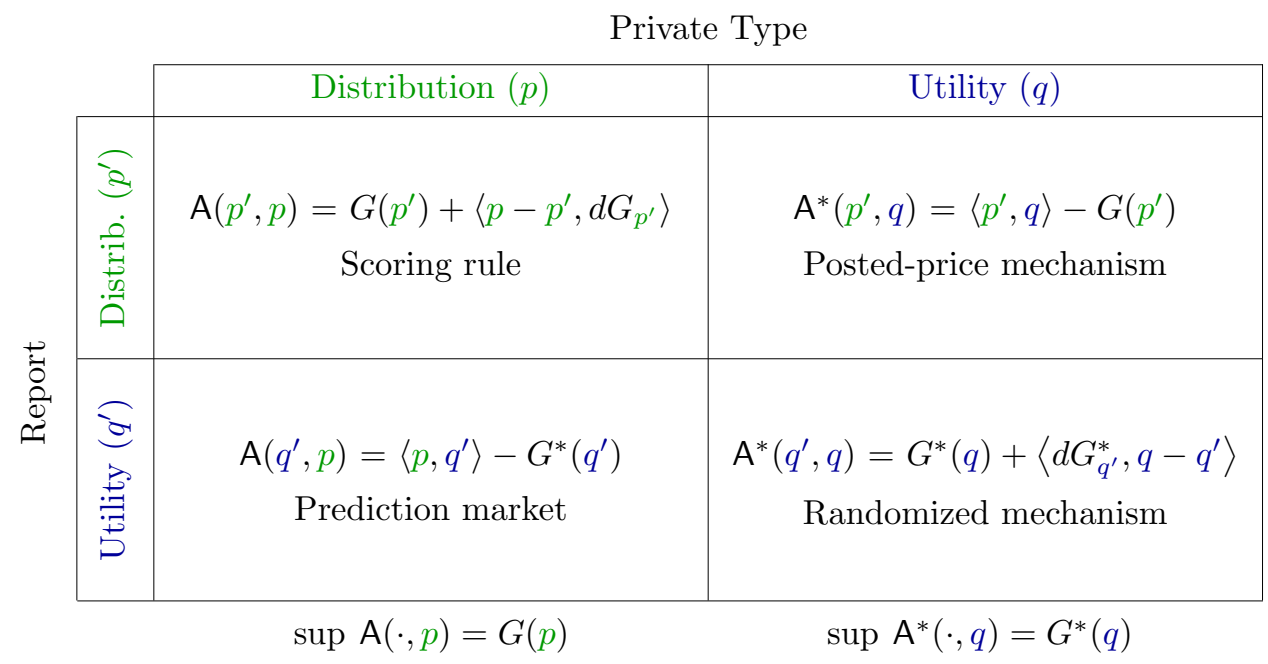

Table I: The duality quadrangle for the duality between distributions and functionals.

Fix a finite outcome space $\mathcal{O}$. Let $\mathcal{S}$ be the set of all scoring rules $\mathrm{S}: \mathcal{P} \times \mathcal{O} \rightarrow \overline{\mathbb{R}}$ for some convex set of distributions $\mathcal{P} \subseteq \Delta(\mathcal{O})$, and $\mathcal{M}$ the set of all randomized mechanisms $(f: \mathcal{T} \rightarrow \Delta(\mathcal{O}), \pi: \mathcal{T} \rightarrow \mathbb{R})$ for some convex type space $\mathcal{T} \subseteq \mathbb{R}^{\mathcal{O}}$. (We use $\pi$ for prices to avoid confusion with probabilities.) In what follows, we will write expectations as dot products, such as $t \cdot f\left(t^{\prime}\right)=\mathbb{E}_{o \sim f\left(t^{\prime}\right)}[t(o)]$.

Construction 1. Given a scoring rule $\mathrm{S}: \mathcal{P} \times \mathcal{O} \rightarrow \overline{\mathbb{R}}$, let $G_{\mathrm{S}}: \mathcal{P} \rightarrow \overline{\mathbb{R}}$ be defined by $G_{\mathbf{S}}(p)=\sup _{p^{\prime} \in \mathcal{P}} \mathrm{S}\left(p^{\prime}, p\right)$. Similarly, given a randomized mechanism $\mathrm{M}=(f, \pi)$ on type space $\mathcal{T}$, let $G_{\mathrm{M}}: \mathcal{T} \rightarrow \overline{\mathbb{R}}$ be given by $G_{\mathrm{M}}(t)=\sup _{t^{\prime} \in \mathcal{T}} U\left(t^{\prime}, t\right)=\sum_{t^{\prime} \in \mathcal{T}} t \cdot f\left(t^{\prime}\right)-\pi\left(t^{\prime}\right)$. Define the multivalued maps $\Phi: \mathcal{S} \rightrightarrows \mathcal{M}$ and $\Psi: \mathcal{M} \rightrightarrows \mathcal{S}$ as follows,

$$
\begin{aligned}
\Phi(\mathrm{S}) & =\left\{\mathrm{M}=(f, \pi) \mid \forall t \in \mathcal{T} f(t) \in \partial G_{\mathrm{S}}^{*}(t), \pi(t)=t \cdot f(t)-G_{\mathrm{S}}^{*}(t)\right\} \\
\Psi(\mathrm{M}) & =\left\{\mathrm{S}(p, o)=G_{\mathrm{M}}^{*}(p)+d_{p}(o)-d_{p} \cdot p \mid \forall p \in \mathcal{P} d \in \partial G_{\mathrm{M}}^{*}(p)\right\},
\end{aligned}
$$

where for any function $F: D \rightarrow \overline{\mathbb{R}}$ on domain $D \subseteq \mathbb{R}^{d}$ we write $F^{*}: \mathbb{R}^{d} \rightarrow \overline{\mathbb{R}}$ for the convex conjugate, $F^{*}(x)=\sup _{d \in D} d \cdot x-F(d)$.

Our construction takes a scoring rule and produces a set of mechanisms, and vice versa. We now show that these are inverse operations, under mild regularity assumptions. The set-valued nature of our construction merely follows from the choices in selecting subgradients in Theorem 2.3 as we show in part (3) below, when there is only one choice for the subgradients, the maps $\Phi, \Psi$ become single-valued.

THEOREM 5.6. We have the following:

(1) For any regular proper scoring rule $\mathrm{S}$, any $\mathrm{M} \in \Phi(\mathrm{S})$ is a truthful mechanism; conversely, for any truthful mechanism $\mathrm{M}$, any $\mathrm{S} \in \Psi(\mathrm{M})$ is a regular proper scoring rule.

(2) Let $\mathcal{C}$ be the set of closed convex functions. Restricting to the sets $\left\{\mathrm{S} \in \mathcal{S}: G_{\mathrm{S}} \in \mathcal{C}\right\}$ and $\left\{\mathrm{M} \in \mathcal{M}: G_{\mathrm{M}} \in \mathcal{C}\right\}$, the maps $\Phi$ and $\Psi$ are inverses as multivalued maps.

(3) Let $\mathcal{D}$ the set of closed strictly convex functions $f$ which are differentiable in the interior $I$ of their domain, and satisfy $\lim _{k \rightarrow \infty}\left\|\nabla f\left(x_{k}\right)\right\|=\infty$ for any sequence $x_{1}, x_{2}, \ldots \in I$

${ }^{6} \mathrm{~A}$ convex function is closed if its epigraph is a closed set, or equivalently, if it is lower semi-continuous. 
converging to a boundary point of I. Restricting to the sets $\left\{\mathrm{S} \in \mathcal{S}: G_{\mathrm{S}} \in \mathcal{D}\right\}$ and $\left\{\mathrm{M} \in \mathcal{M}: G_{\mathrm{M}} \in \mathcal{D}\right\}, \Phi$ and $\Psi$ are single-valued (and inverses). Furthermore, every scoring rule and mechanism in these sets is strictly proper/truthful.

Proof. For part (1), note that by Corollaries 2.5 and 2.10, the forms are proper/truthful if we can show that the convex functions are finite on the correct domain, and subdifferentiable in the case of $G_{\mathrm{S}}^{*}$ (recall that we allow vertical subgradients for scoring rules). First, let $\mathrm{S}$ be given. Let $\mathcal{T}=\partial G_{\mathrm{S}}(\mathcal{P}) \cap \mathbb{R}^{\mathcal{O}}$ be the set of finite subgradients, which is a convex set. From Rockafellar 1997, Corollary 13.3.1] we have that $G_{\mathrm{S}}^{*}$ is finite on all of $\mathbb{R}^{\mathcal{O}}$ as the domain of $G_{\mathrm{S}}$ is bounded. (The Corollary requires $G_{\mathrm{S}}$ to be closed, but we also have $G_{\mathrm{S}}^{*}=\left(\mathrm{cl} G_{\mathrm{S}}\right)^{*}$, and the domain of $\mathrm{cl} G_{S}$ is still bounded, where cl denotes the closure of a function.) Subdifferentiability of $G_{\mathrm{S}}^{*}$ is then implied by Rockafellar 1997, Theorem 23.4]. Conversely, given some mechanism $\mathrm{M}$, we can show that that $G_{\mathrm{M}}^{*}$ is finite on $\mathcal{P}=\partial G_{\mathrm{M}}(\mathcal{T}) \cap \Delta(\mathcal{O})$. Applying Rockafellar 1997, Theorem 23.5], we have $p \in \partial G_{\mathrm{M}}(t) \Longleftrightarrow G_{\mathrm{M}}(t)+G_{\mathrm{M}}^{*}(p)=p \cdot t$, and as $p \cdot t$ and $G_{\mathrm{M}}(t)$ are both finite, we conclude that $G_{\mathrm{M}}^{*}(p)$ is finite. Thus, by the Corollaries, we are done.

For (2), note that $G_{\mathrm{S}}^{*}$ and $G_{\mathrm{M}}^{*}$ are closed and convex, and we have both $\left(G_{\mathrm{S}}^{*}\right)^{*}=G_{\mathrm{S}}$ and $\left(G_{\mathrm{M}}^{*}\right)^{*}=G_{\mathrm{M}}$ Rockafellar 1997, Theorem 12.2]. The inverse relationship then follows from the fact that the multivalued maps 20 depend only on $G_{\mathrm{S}}$ and $G_{\mathrm{M}}$, and every scoring rule / mechanism in the range shares the same expected score / consumer surplus function.

For (3), we simply appeal to Rockafellar 1997, Theorem 26.3 \& Corollary 26.3.1]. Strictness follows from strict convexity.

To illustrate Theorem 5.6. consider the $\log$ scoring rule $\mathrm{S}(p, o)=\log p(o)$, which is strictly proper. The expected score function is negative Shannon entropy, $G_{\mathbf{S}}(p)=$ $\sum_{o \in \mathcal{O}} p(o) \log p(o)$, which satisfies the conditions of part (3) of Theorem 5.6 Applying $\Phi$, we therefore obtain a unique mechanism, which chooses the allocation probabilites according to the familiar multiplicative weights formula:

$$
f(t)=\left(\frac{e^{t(o)}}{\sum_{o^{\prime} \in \mathcal{O}} e^{t\left(o^{\prime}\right)}}\right)_{o \in \mathcal{O}} \in \Delta(\mathcal{O}), \quad \pi(t)=\log \sum_{o \in \mathcal{O}} e^{t(o)}-\frac{\sum_{o \in \mathcal{O}} t(o) e^{t(o)}}{\sum_{o \in \mathcal{O}} e^{t(o)}} .
$$

The prices $\pi$ bear resemblance to the Log Market Scoring Rule (LMSR) Hanson 2003, but as remarked above, note that the private information here is a fixed valuation function $t$, not a belief in the form of a probability distribution.

The bijection in Theorem 5.6 is not an arbitrary one; a mechanism and scoring rule which are related by $\Phi$ or $\Psi$ satisfy certain identities. For example, for $S$ and $M=(f, \pi)$ satisfying part (2) of the theorem, and $M \in \Phi(S)$, a standard result of convex analysis states that $G_{\mathrm{S}}(p)+G_{\mathrm{M}}(t)=p \cdot t$ whenever either $t=d$ (from eq. (20p) or $p=f(t)$ hold. To provide further intuition for this relationship with a somewhat whimsical example, suppose a gambler in a casino examines the rules of a dice-based game of chance and forms belief $p$ about the probabilities of possible outcomes, assuming the dice are fair. The gambler is then offered a proper scoring rule $S$ to predict the outcome of the game, and reports truthfully. Before the game is played, however, the casino informs the gambler that the dice used need not be fair, and offers the gambler the opportunity to select from among different choices of dice using a truthful mechanism $\mathrm{M}$ where the gambler's private information is the valuation function $t$ given by $t(o)=\mathrm{S}(p, o) \cdot]^{7}$ note that $t(o)$ truly is the gambler's valuation of outcome $o$. If $\mathrm{M} \in \Phi(\mathrm{S})$, and the gambler again reports truthfully, then the dice chosen

\footnotetext{
${ }^{7}$ Strictly speaking, to directly apply the convex analysis result referenced above, we would need to take $t(o)$ to be the linear part of $\mathrm{S}(p, o)$; in our setting, however, the allocation and prices of the mechanism are invariant to adding a constant to the valuation of every outcome. (To see this, note that the subgradients of $G_{\mathrm{M}}=G_{\mathrm{S}}^{*}$ are probability distributions, so the directional derivative is 1 in the direction of $\mathbb{1}$, the all-ones vector; integrating gives the result. See also Abernethy et al. 2013.)
} 
by the mechanism will be fair. And what will be the gambler's profit in expectation from both the scoring rule and mechanism? Zero. This follows from the above identity, and the observation that $t \cdot f(t)-\pi(t)=\mathrm{S}(p, p)-\left(p \cdot t-G_{\mathrm{M}}(t)\right)=G_{\mathrm{S}}(p)+G_{\mathrm{M}}(t)-p \cdot t$. 7 The power of our construction is that these relationships hold regardless of the initial scoring rule $S$.

\section{FINITE-VALUED PROPERTIES}

We now examine the special case where $\mathcal{R}$ is a finite set of reports, using the additional structure to provide stronger characterizations. In the scoring rules literature, Lambert and Shoham 2009 view this as eliciting answers to multiple-choice questions. There are also applications to mechanism design, discussed in Section 6.1. Assume throughout that $\mathcal{R}$ is finite and that $\mathcal{T}$ is a convex subset of a Hilbert space $\mathcal{V}\left(\right.$ e.g., $\left.\mathcal{V}=\mathbb{R}^{d}\right)$ with inner product written $\left\langle t, t^{\prime}\right\rangle$ and norm $\|t\|^{2}=\langle t, t\rangle$. In this setting, we will use the concept of a power diagram from computational geometry.

Definition 6.1. Given a set of points $P=\left\{p_{i}\right\}_{i=1}^{m} \subset \mathcal{V}$, called sites, and weights $w \in \mathbb{R}^{m}$, a power diagram $D(P, w)$ is a collection of cells cell $\left(p_{i}\right) \subseteq \mathcal{T}$ defined by

$$
\operatorname{cell}_{P, w}\left(p_{i}\right)=\left\{t \in \mathcal{T} \mid i \in \underset{j}{\operatorname{argmin}}\left\{\left\|p_{j}-t\right\|^{2}-w_{j}\right\}\right\} .
$$

The following result is a straightforward generalization of Theorem 4.1 of Lambert and Shoham 2009, and is essentially a restatement of results due to Aurenhammer 1987a; $1987 \mathrm{~b}$.

TheOREM 6.2. A property $\Gamma: \mathcal{T} \rightrightarrows \mathcal{R}$ for finite $\mathcal{R}$ is elicitable if and only if the level sets $\left\{\Gamma_{r}\right\}_{r \in \mathcal{R}}$ form a power diagram $D(P, w)$.

Proof. Let us examine the condition that $t$ is an element of $\operatorname{cell}_{P, w}\left(p_{i}\right)$ for some power diagram $D(P, w)$ :

$$
\begin{aligned}
t \in \operatorname{cell}_{P, w}\left(p_{i}\right) & \Longleftrightarrow i \in \underset{j}{\operatorname{argmin}}\left\{\left\|p_{j}-t\right\|^{2}-w_{j}\right\} \\
& \Longleftrightarrow i \in \underset{j}{\operatorname{argmin}}\left\{\left\|p_{j}\right\|^{2}-2\left\langle p_{j}, t\right\rangle-w_{j}\right\} .
\end{aligned}
$$

Note that eq. 23) is affine in $t$. Now given some $D=D(P, w)$ with index set $\mathcal{R}$, we simply let $\mathrm{A}(r, t)=2\left\langle p_{r}, t\right\rangle+w_{r}-\left\|p_{r}\right\|^{2}$. By (23) we immediately have $r \in \operatorname{argsup}_{r^{\prime}} \mathrm{A}\left(r^{\prime}, t\right) \Longleftrightarrow$ $t \in \operatorname{cell}_{P, w}\left(p_{r}\right)$, as desired.

Conversely, let an affine score $A$ eliciting $\Gamma$ be given. By the Fréchet-Riesz representation theorem, we may write $\mathrm{A}(r, t)=\left\langle x_{r}, t\right\rangle+c_{r}$ for $x_{r} \in \mathcal{V}$ and $c_{r} \in \mathbb{R}$. Letting $p_{r}=x_{r} / 2$ and $w_{r}=\left\|p_{r}\right\|^{2}+c_{r}$, we see by 23 again that $\Gamma_{r}=\operatorname{cell}\left(p_{r}\right)$ of the diagram $D\left(\left\{p_{r}\right\}, w\right)$. Hence, $\Gamma$ is a power diagram.

We have now seen what kinds of finite-valued properties are elicitable, but how can we elicit them? More precisely, as the proof above gives sufficient conditions, what are all ways of eliciting a given power-diagram? In general, it is difficult to provide a "closed form" answer to this question, so we restrict to the simple case, where essentially the cells of a power diagram are as constrained as possible.

Definition 6.3 ( (Aurenhammer 198\%c/). A $j$-polyhedron is the intersection of dimension $j$ of a finite number of closed halfspaces of $\mathcal{V}=\mathbb{R}^{d}$, where $0 \leq j \leq d$. A tiling $C$ in $\mathcal{V}$ is a covering of $\mathcal{V}$ by finitely many $j$-polyhedra, called $j$-faces of $C$, whose (relative) interiors are disjoint. If furthermore their non-empty intersections are faces of $C$ then $C$ is a cell complex. A cell complex $C$ is called simple if each of its $j$-faces is in the closure of exactly $(d-j+1) d$-faces (cells). 
TheOREM 6.4. Let $\mathcal{V}=\mathbb{R}^{d}$ and let finite-valued, elicitable, simple property $\Gamma: \mathcal{T} \rightrightarrows \mathcal{R}$ be given. Then there exist points $\left\{p_{r}\right\}_{\mathcal{R}} \subseteq \mathcal{V}$ such that the following holds: for any affine score $\mathrm{A}: \mathcal{R} \times \mathcal{T} \rightarrow \overline{\mathbb{R}}$ eliciting $\Gamma$, there exist $\alpha>0, p_{0} \in \mathcal{V}$, and $w \in \mathbb{R}^{\mathcal{R}}$ such that

$$
\mathrm{A}(r, t)=2\left\langle\alpha p_{r}+p_{0}, t\right\rangle-\left\|\alpha p_{r}+p_{0}\right\|^{2}+w_{r},
$$

and conversely, for all such $\alpha$ and $p_{0}$ there exists $w \in \mathbb{R}^{\mathcal{R}}$ making $\mathrm{A}$ in eq. 24) elicit $\Gamma$.

Proof. A result of Aurenhammer for simple cell complexes, given in Lemma 1 of $\mathrm{Au}-$ renhammer 1987b and the proof of Lemma 4 of Aurenhammer 1987a, states the following: given sites $P$ and $P^{\prime}$ and weights $w$, there exist weights $w^{\prime}$ such that $D\left(P^{\prime}, w^{\prime}\right)=D(P, w)$ if and only if $P^{\prime}$ is a homothet (translated and positively scaled copy) of $P$. We simply apply this fact to the proof of Theorem 6.2.

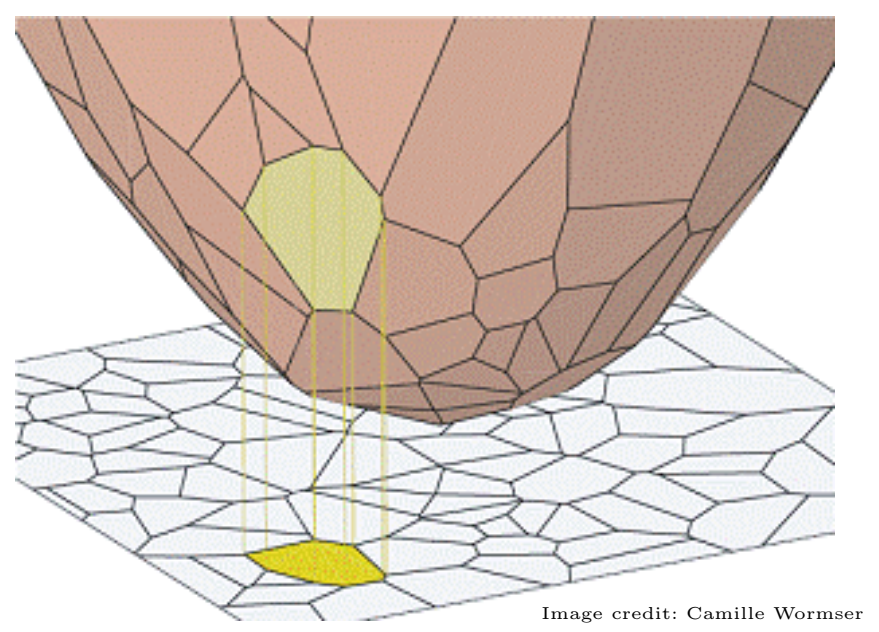

Fig. 2: A consumer surplus function $G$ and its corresponding partition of the type space, $\Gamma$. The proof of Theorem 6.2 leverages the fundamental relationship between projections of convex functions and power diagrams.

See Appendix E for a discussion about Bregman Voronoi digrams and the role of $\|\cdot\|^{2}$ in Theorem 6.2

Detecting elicitable finite properties. As a practical matter, it is natural to ask if we can efficiently determine whether a given finite-valued property $\Gamma$ is elicitable. By Theorem 6.2 , we need only test whether the cells $C=\left\{\Gamma_{r}\right\}_{r \in \mathcal{R}}$ form a power diagram. For the simple case, Aurenhammer gives the "Orthogonal Dual" algorithm for this task; see $\S 2.2$ of $\overline{\mathrm{Au}}-$ renhammer $1987 \mathrm{c}$ and comments thereafter. The orthogonal dual algorithm assumes that the cells are stored in an incidence lattice, with nodes for each face of $C$, and edges when faces are incident (a $j$-dimensional face which contains a $(j-1)$-dimensional face). The runtime of the algorithm is $O(m)$, where $m$ is the number of facets (faces of dimension $d-1$ ). More generally, Borgwardt and Frongillo 2019 present a weakly polynomial-time algorithm to detect power diagrams in the general case, via a simple linear program.

\subsection{Finite Properties in Mechanism Design}

Mechanisms with a finite set of allocations are common. Carroll 2012 examines them and observes they give rise to polyhedral typespaces. Theorem 6.2 strengthens this characterization to power diagrams, which rules out polyhedral examples such as the one shown in 

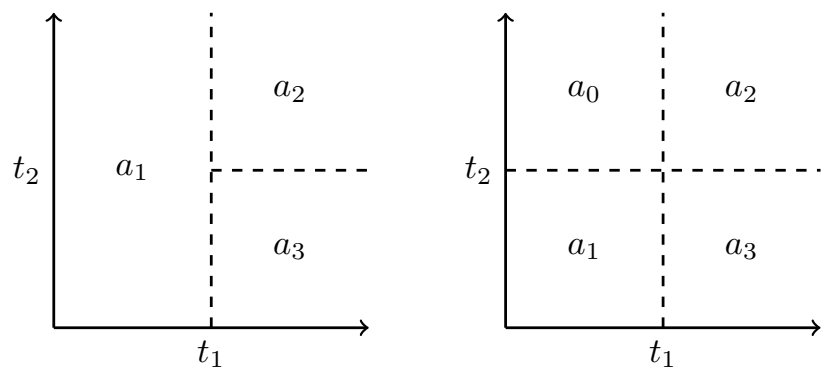

Fig. 3: An allocation rule which cannot be implemented, for any distinct choices of $a_{i}$ (left), and a rule which could be implemented for appropriate choices of $a_{i}$ (right).

Figure 3. In particular, the example of the left of the figure fails to be a power diagram because all power diagrams in $\mathbb{R}^{d}$ are cell complexes Aurenhammer 1987a, while it is merely a tiling as the intersection of the $a_{1}$ and $a_{2}$ cells is not a face of the $a_{1}$ cell.

Suppose we are in a such a mechanism design setting with a finite set of allocations $\mathcal{X}$ and we have picked an allocation rule $a$. Under what circumstances is $a$ implementable, i.e., when is there a payment rule that makes the resulting mechanism truthful? For convex type sets, Saks and $\mathrm{Yu}$ [2005] showed that the following condition is necessary and sufficient.

Definition 6.5. Allocation rule $a$ satisfies weak monotonicity (WMON) if $a(t) \cdot\left(t^{\prime}-t\right) \leq$ $a\left(t^{\prime}\right) \cdot\left(t^{\prime}-t\right)$ for all $t, t^{\prime} \in \mathcal{T}$.

From Theorem 2.3, we know that $a$ being implementable means that there exists a $G$ such that $a$ is a selection of its subgradients. But this is equivalent to saying that the property $\Gamma(t)=\mathcal{X} \cap d G_{t}$ is subgradient-elicitable. This gives us a new proof of this theorem by showing that WMON characterizes power diagrams. In particular, we can leverage the following characterization of power diagrams due to Aurenhammer 1987a. This result assumes that $a$ is defined on all of $\mathcal{V}=\mathbb{R}^{d}$, as until recently power diagrams have not been studied on restricted domains. However, recent results for restricted domains imply that Aurenhammer's argument generalizes in a straightforward way Borgwardt and Frongillo 2019. For completeness, we provide a sketch of the proof for the restricted version.

Definition 6.6. A tiling $C$ of a convex set $\mathcal{T} \subseteq \mathcal{V}$ is a covering of $\mathcal{T}$ by finitely many $j$-polyhedra, called $j$-faces of $C$, whose (relative) interiors when restricted to $\mathcal{T}$ are disjoint. $\mathbb{R}^{d}$.

Theorem 6.7 (|AUREnhammer 1987A]). Let $C$ be a tiling of a convex set $\mathcal{T} \subseteq \mathcal{V}=$

Then $C$ is the restriction to $\mathcal{T}$ of a power diagram defined on all of $\mathcal{V}$, if and only if there exists a point-set $\left\{p_{1}, \ldots, p_{n}\right\}$ satisfying,

(1) Orthogonality: For $Z_{i} \neq Z_{j}$, the line $L$ that contains $p_{i}$ and $p_{j}$ (and is directed from $p_{i}$ to $p_{j}$ ) is orthogonal to each face common to $Z_{i}$ and $Z_{j}$.

(2) Orientation: Any directed line that can be obtained by translating $L$ and that intersects $Z_{i}$ and $Z_{j}$ first meets $Z_{i}$.

Proof. One direction is trivial: if $C$ is a restriction of a power diagram to $\mathcal{T}$ then by Aurenhammer's original theorem the unrestricted power diagram has such a point set which satisfies orthogonality and orientation on all of $\mathcal{V}$ and thus also on $\mathcal{T}$. For the other direction, Borgwardt and Frongillo 2019 show that a tiling of $\mathcal{T}$ is a restriction of a power diagram if and only if the following LP is feasible: 


$$
\begin{aligned}
\lambda_{i j} \cdot a_{i j} & =p_{i}-p_{j} & & \forall i \leq k, \forall j \in J_{i} \\
\lambda_{i j} \cdot \gamma_{i j} & =\gamma_{j}-\gamma_{i} & & \forall i \leq k, \forall j \in J_{i} \\
\lambda_{i j} & >0 & & \forall i \leq k, \forall j \in J_{i}
\end{aligned}
$$

Here $i, j \in\{1, \ldots, n\}$ index into the cells, $J_{i}$ is the indices of cells adjacent to cell $i$, and the cells are given by constants $a_{i j} \in \mathbb{R}^{d}, \gamma_{i j} \in \mathbb{R}$, so that the $i$ th cell is defined as $\left\{t \in \mathcal{T} \mid a_{i j} \cdot t \leq \gamma_{i j} \forall j \in J_{i}\right\}$. Thus, the variables are the sites $\left\{p_{1}, \ldots, p_{n}\right\} \subseteq \mathbb{R}^{d}$ and pseudo-weights $\left\{\gamma_{1}, \ldots, \gamma_{n}\right\} \subseteq \mathbb{R}$ which are in bijection with the true weights.

In our setting, by contrast, we are given the $p_{i}$ along with the $a_{i j}$ and $\gamma_{i j}$, and need only find real numbers $\gamma_{i}$ and $\lambda_{i j}$ for which the program is feasible. By orthogonality, there is a unique choice of $\lambda_{i j}$ satisfying the first constraint. Furthermore, by orientation it is strictly positive, satisfying the third constraint. For the second constraint, for any pair $(i, j)$ with $j \in J_{i}$ and choice of $\gamma_{i}$ there is a unique $\gamma_{j}$ satisfying the constraint. Establishing the existence of a globally consistent set of choices is the heart of Aurenhammer's argument. In particular, he shows that if $i, j, k$ share a vertex of $C$ then for arbitrary $\gamma_{i}$ the unique choices of $\gamma_{j}$ and $\gamma_{k}$ which satisfy the second constraint for $(i, j)$ and $(i, k)$ also satisfy it for $(j, k)$. Global consistency then follows by a simple inductive construction. Start by choosing a cell $i$ and arbitrary $\gamma_{i}$. At each step we assign some $\gamma_{j}$. If there is a $j$ for which $\gamma_{j}$ is unassigned and $j$ has a vertex which is shared with two assigned cells then, per Aurenhammer's argument, we can assign $\gamma_{j}$ consistent with all cells assigned so far. Otherwise every unassigned cell has a most a single face in common with a single assigned cell and therefore we can choose one which has such a face and it can trivially be assigned consistently.

TheOREM 6.8. A tiling $C$ is a power diagram with sites $\left\{p_{1}, \ldots, p_{n}\right\}$ if and only if for all $t \in Z_{i}$ and $t^{\prime} \in Z_{j}$ we have $p_{i} \cdot\left(t^{\prime}-t\right) \leq p_{j} \cdot\left(t^{\prime}-t\right)$ (i.e. C satisfies WMON).

Proof. If $C$ is a power diagram, then by definition

$$
\begin{aligned}
2 p_{i} \cdot t-w_{i} & \geq 2 p_{j} \cdot t-w_{j} \\
2 p_{j} \cdot t^{\prime}-w_{j} & \geq 2 p_{i} \cdot t^{\prime}-w_{i} .
\end{aligned}
$$

Adding these shows $C$ satisfies WMON.

Now suppose $C$ satisfies WMON. We show orthogonality and orientation. For orthogonality, let $t, t^{\prime} \in Z_{i} \cap Z_{j}$. Then $p_{i} \cdot\left(t^{\prime}-t\right)=p_{j} \cdot\left(t^{\prime}-t\right)$, or $\left(p_{i}-p_{j}\right) \cdot\left(t^{\prime}-t\right)=0$. Thus, the face is orthogonal to $L$. For orientation, let $t \in Z_{i}$ and $t^{\prime} \in Z_{j}$ be on such a translated $L$. That is, we can write $t^{\prime}=t+c\left(p_{j}-p_{i}\right)$ for some $c \in \mathbb{R}$. By WMON, $\left(p_{j}-p_{i}\right) \cdot\left(t^{\prime}-t\right) \geq 0$, or $c\left(p_{j}-p_{i}\right) \cdot\left(p_{j}-p_{i}\right) \geq 0$. Thus $c \geq 0$. Therefore such a translated $L$ first meets $Z_{i}$.

Corollary 6.9 ([SAKS AND YU 2005]). If $\mathcal{X}$ is finite, $\mathcal{T}$ is convex, and a satisfies WMON, then a is implementable.

Proof. In order to apply Theorems 6.2 and 6.8 it remains to show that an allocation rule $a$ satisfying WMON further implies that it defines a tiling. This follows by a straightforward geometric argument that has been used in a number of previous proofs (see, e.g., Lemma 4.2 of Archer and Kleinberg 2008). For completeness, provide it here.

Let $x \in \mathcal{X}$ be given. We can define a polyhedron $P_{x}$ associated with $x$ by the intersection of

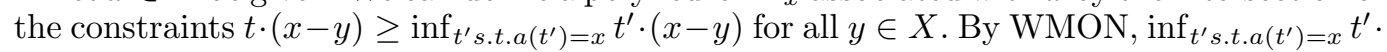
$(x-y) \geq \sup _{\text {ts.t.a }(t)=y} t \cdot(x-y)$, so for any distinct $x, y \in \mathcal{X}, P_{x}$ and $P_{y}$ are separated by the hyperplane $t \cdot(x-y)=\inf _{t^{\prime} \text { s.t.a } a\left(t^{\prime}\right)=x} t^{\prime} \cdot(x-y)$, so their (relative) interiors are disjoint. By construction $t \in P_{a(t)}$ for all $t$, which implies that these polyhedra cover $\mathcal{T}$.

\section{DISCUSSION}

We have presented a model of truthful elicitation which generalizes and extends both mechanisms and scoring rules. On the mechanism design side, we have seen how our framework 
provides simpler, more general, or more constructive proofs of a number of known results about implementability and revenue equivalence, some of which lead to new results about scoring rules. On the scoring rules side, we have provided the first characterization for scoring rules for non-convex sets of probability distributions. We have also extended our model to eliciting a property of the agent's private information. This has been studied for specific cases in the scoring rules literature, but we have provided the first general characterization. We also show how results about power diagrams in the scoring rules literature lead to a new proof of the Saks-Yu result in mechanism design.

Our analysis makes use of the fact that $\mathrm{A}\left(t^{\prime}, t\right)$ is affine in $t$ to ensure that $G(t)=$ $\sup _{t^{\prime}} \mathrm{A}\left(t^{\prime}, t\right)$ is a convex function. However, this property continues to hold if $\mathrm{A}\left(t^{\prime}, t\right)$ is instead a convex function of $t$. Thus, a natural direction for future work is to investigate characterizations of convex scores. While mechanisms can always be represented as affine functions by taking the types to be functions from allocations to $\mathbb{R}$, it may be more natural to treat the type as a parameter of a (convex) utility function. While many such utility functions are affine (e.g. dot-product valuations), others such as Cobb-Douglas functions are not. Berger, Müller, and Naeemi 2009, 2010 have investigated such functions and given characterizations that suggest a more general result is possible. Another potential application is scoring rules for alternate representations of uncertainty, several of which result in a decision maker optimizing a convex function Halpern 2003.

In one sense getting such a characterization is straightforward. In the affine case we want $\mathrm{A}\left(t^{\prime}, t\right)$ to be an affine function such that $\mathrm{A}\left(t^{\prime}, t\right) \leq G(t)$ and $\mathrm{A}\left(t^{\prime}, t^{\prime}\right)=G\left(t^{\prime}\right)$. Since we have fixed its value at a point, the only freedom we have is in the linear part of the function, and being such a linear function is exactly the definition of a subgradient. So while our characterization of affine scores is in some sense vacuous, it is also powerful in that it allows us to make use of the tools of convex analysis. A similarly vacuous characterization is possible for the convex case: $\mathrm{A}\left(t^{\prime}, t\right)$ is a convex function such that $\mathrm{A}\left(t^{\prime}, t\right) \leq G(t)$ and $\mathrm{A}\left(t^{\prime}, t^{\prime}\right)=G\left(t^{\prime}\right)$. The challenge is to find a way to state it that is useful and naturally handles constraints such as those imposed by the form of a utility function.

Many questions in the literature on properties remain open. Most notable is the characterization of elicitable nonlinear and multidimensional properties - the single dimensional case is covered in Lambert et al. 2008 and the linear vector-valued case in Abernethy and Frongillo 2012. We hope that the results and intuition from Section 4 will yield a useful characterization in this case. Subsequent to this work we have made some partial progress on this approach Frongillo and Kash 2015a. Another interesting direction is for non-functional properties: aside from the finite $R$ case, all work in the literature to our knowledge assumes that $\Gamma$ is a function (having a single correct report for each type). The generality of Theorem 4.5 may prove useful in exploring non-functional settings as well. A result requiring few regularity conditions on $\Gamma$ would be useful in domains such as statistics where natural properties like the median cannot in general be expressed as functions.

Theorem 6.2 shows that scoring rules for finite properties are essentially equivalent the weights and points that induce a power diagram. As power diagrams are known to be connected to the spines of amoebas in algebraic geometry, aspects of toric geometry used by string theorists, and tropical hypersurfaces in tropical geometry Van Manen and Siersma 2005, there may be useful characterization results in those fields as well. The last is particularly suggestive given the recent use of tropical geometry techniques in mechanism design Baldwin and Klemperer 2012].

While our examples have focused on mechanism design and scoring rules, another interesting direction to pursue is other settings where our results may be applicable. One natural domain is the literature on M-estimators in machine learning, statistics and economics. Essentially, this literature takes a loss function (i.e. a scoring rule) and asks what it elicits. For example, the mean is an M-estimator induced by the squared error loss function. Some work in this literature (e.g. [Negahban et al. 2010]) requires that the loss function satisfy 
certain conditions, and our results may be useful in characterizing and supplying such loss functions.

\section{A. CONVEX ANALYSIS PRIMER}

In this appendix, we review some facts from convex analysis that are used in the paper.

FACT 1. Let $\left\{f_{t} \in \operatorname{Aff}(\mathcal{V} \rightarrow \mathbb{R})\right\}_{t \in \mathcal{T}}$ be a parameterized family of affine functions. Then $G(t)=\sup _{t^{\prime} \in T} f_{t^{\prime}}(t)$ is convex as the pointwise supremum of convex functions.

This follows because convex functions are those with convex epigraphs. The epigraph of this supremum is the intersection of the epigraphs of the individual functions, which is a convex set as the intersection of convex sets.

FACT 2. $d: \mathbb{R} \rightarrow \mathbb{R}$ is a selection of subgradients of a convex function on $\mathbb{R}$ if and only if it is monotone non-decreasing.

See Rockafellar 1997, Theorem 24.3] for a proof of a slightly more general statement.

FaCt 3. For convex $G$ on convex $\mathcal{T},\left\{d G_{t} \in \operatorname{Lin}(\mathcal{V} \rightarrow \mathbb{R})\right\}_{t \in \mathcal{T}} \in \partial G$ satisfies path independence.

Informally, path independence means that integrals of $d G_{t}$ do not depend on the path through $\mathcal{T}$ chosen (see Equation (30) and following for a formal definition). Since the restriction of $G$ to a line is a one-dimensional convex function, $G(y)-G(x)=\int_{L_{x y}} d G_{t}(y-$ $x) d t$ [Rockafellar 1997, Corollary 24.2.1]. Summing along the individual lines in a path from $x$ to $y$ gives that the value of the path integral is $G(y)-G(x)$ regardless of the path chosen.

The following is a classic result in convex analysis (cf. Urruty and Lemaréchal 2001, Thm E.1.4.1]) which we prove for completeness.

FACT 4. Let $G: \mathcal{V} \rightarrow \overline{\mathbb{R}}$ be convex. Then for all $v \in \mathcal{V}, d \in \mathcal{V}^{*}$,

$$
G^{*}(d)=d(v)-G(v) \Longleftrightarrow d \in \partial G_{v} .
$$

Proof. We can simply break down the conditions step by step:

$$
\begin{aligned}
G^{*}(d)=d(v)-G(v) & \Longleftrightarrow v \in \operatorname{argsup}_{v^{\prime} \in \mathcal{V}} d\left(v^{\prime}\right)-G\left(v^{\prime}\right) \\
& \Longleftrightarrow \forall v^{\prime} \in \mathcal{V}, d(v)-G(v) \geq d\left(v^{\prime}\right)-G\left(v^{\prime}\right) \\
& \Longleftrightarrow \forall v^{\prime} \in \mathcal{V}, G\left(v^{\prime}\right) \geq G(v)+d\left(v^{\prime}-v\right),
\end{aligned}
$$

where in the last step we merely negated and added $d\left(v^{\prime}\right) \in \mathbb{R}$ to both sides.

FACT 5. For any convex function $G$, the set $\partial G^{-1}(d) \doteq\left\{x \in \operatorname{dom}(G): d \in \partial G_{x}\right\}$ is convex.

Proof. Let $x, x^{\prime} \in \partial G^{-1}(d)$; then one easily shows (cf. Lemma 4.4 that $G(x)-G\left(x^{\prime}\right)=$ $d\left(x-x^{\prime}\right)$. Now let $\hat{x}=\alpha x+(1-\alpha) x^{\prime} ;$ we have,

$$
\begin{aligned}
G(\hat{x}) & \leq \alpha G(x)+(1-\alpha) G\left(x^{\prime}\right) \\
& =\alpha\left(G(x)-G\left(x^{\prime}\right)\right)+G\left(x^{\prime}\right) \\
& =\alpha d\left(x-x^{\prime}\right)+G\left(x^{\prime}\right) \\
& =d\left(\hat{x}-x^{\prime}\right)+G\left(x^{\prime}\right) \\
& \leq G(\hat{x}),
\end{aligned}
$$

where we applied convexity of $G$ in 25 and the subgradient inequality for $d$ at $x^{\prime}$ in (27). Hence, by eq. 26, we have shown $G(\hat{x})-G\left(x^{\prime}\right)=d\left(\hat{x}-x^{\prime}\right)$, so by Lemma $4.4, d \in \partial G_{\hat{x}}$. 


\section{B. CHARACTERIZING TRUTHFUL MECHANISMS}

While our theorem provides a characterization of truthful mechanisms in terms of convex consumer surplus functions, this is not always the most natural representation for a mechanism. In this section, we examine two other approaches to characterizing truthful mechanisms that have been explored in the literature and show that they have insightful interpretations in convex analysis, which allows us to greatly simplify their proofs. Furthermore, our phrasing of these results is as conditions for a parameterized family of linear functions to be a selection of subgradients of a convex function. We believe this phrasing converts known results in mechanism design into new results in convex analysis. It also shows how any such result in convex analysis would give a characterization of implementable mechanisms. Note that certain results in this section require an assumption that the relevant parameterized families are in fact real-valued, which is natural given our focus on mechanism design.

\section{B.1. Subgradient characterizations}

From an algorithmic perspective, it may be more natural to focus on the design of the allocation rule $f$. There is a large literature that focuses on when there exists a choice of payments $p$ to make $f$ into a truthful mechanism (e.g. Saks and Yu 2005, Ashlagi et al. 2010]). Viewed through our theorem, this becomes a very natural convex analysis question: when is a function $f$ a subgradient of a convex function? 8 Unsurprisingly, the central result in the literature is closely connected to convex analysis.

Definition B.1. A family $\left\{d_{t} \in \operatorname{Lin}(\mathcal{V} \rightarrow \mathbb{R})\right\}_{t \in \mathcal{T}}$ satisfies cyclic monotonicity (CMON) if for all finite sets $\left\{t_{0}, \ldots, t_{k}\right\} \subseteq \mathcal{T}$,

$$
\sum_{i=0}^{k} d_{t_{i}}\left(t_{i+1}-t_{i}\right) \leq 0
$$

where indices are taken modulo $k+1$. The weaker condition that 28) hold for all pairs $\left\{t_{0}, t_{1}\right\}$ is known as weak monotonicity (WMON).

A well known characterization from convex analysis is that a function $f$ defined on a convex set is a subgradient of a convex function on that set iff it satisfies CMON Rockafellar 1997. Rochet's 1987. proof that payments exist to implement $f$ on a possibly non-convex $\mathcal{T}$ iff $f$ satisfies CMON is effectively a proof of a generalization of this theorem. Rochet notes that his proof is adapted from the one given in Rockafellar's text [1997] of the weaker theorem where $\mathcal{T}$ is restricted to be convex. We adapt Rochet's proof to highlight how its core is a construction of $G$. As we use this basic construction several times, we first analyze it independly.

Given any family $\left\{d_{t}\right\}_{t \in \mathcal{T}}$ of linear functions in $\operatorname{Lin}(\mathcal{V} \rightarrow \mathbb{R})$, define $P_{d}: \mathcal{T} \times \mathcal{V} \rightarrow \overline{\mathbb{R}}$ as follows: 9

$$
P_{d}\left(t, t^{\prime}\right) \doteq \sup _{\substack{k \in \mathbb{N},\left\{t_{1}, \ldots, t_{k}\right\} \subseteq \mathcal{T} \\ t_{0}=t, t_{k+1}=t^{\prime}}} \sum_{i=0}^{k} d_{t_{i}}\left(t_{i+1}-t_{i}\right)
$$

One way to interpret $P_{d}\left(t, t^{\prime}\right)$ is as the length of the shortest path from $t$ to $t^{\prime}$ in a graph with edge weights determined by $-d$, and in that form has seen extensive use in mechanism design [Vohra 2011]. We interpret it somewhat differently, as the best lower bound on

\footnotetext{
${ }^{8}$ More precisely, we want for all $t$ the allocation $f(t)$ to be a subgradient at t. Equivalently, we can view $f$ as a parameterized family of functions, which is how we state our results.

${ }^{9}$ Note that the second argument of $P_{d}$ is from $\mathcal{V}$ rather than $T \subset \mathcal{V}$ because we wish to apply this when, e.g., $t^{\prime} \in \operatorname{Conv}(\mathcal{T})$.
} 
$G\left(t^{\prime}\right)-G(t)$ for an arbitrary convex function $G$ with subgradients $d$ (and infinity if there is no such convex function). In particular, computing the best lower bound at every point yields a convex function.

Lemma B.2. Let $\left\{d_{t} \in \operatorname{Lin}(\mathcal{V} \rightarrow \mathbb{R})\right\}_{t \in \mathcal{T}}$ be given. If $d$ satisfies $C M O N$, then for all $t, t^{\prime} \in \mathcal{T}$ and all $t^{\prime \prime} \in \mathcal{V}$, the following hold:

(1) $P_{d}\left(t, t^{\prime}\right)+P_{d}\left(t^{\prime}, t^{\prime \prime}\right) \leq P_{d}\left(t, t^{\prime \prime}\right)$

(2) $d_{t}\left(t^{\prime \prime}-t\right) \leq P_{d}\left(t, t^{\prime \prime}\right)$

(3) $P_{d}(t, t)=0$

(4) $P_{d}\left(t, t^{\prime}\right)+P_{d}\left(t^{\prime}, t\right) \leq 0$

(5) $P_{d}(t, \cdot)$ is convex and real-valued on $\operatorname{Conv}(\mathcal{T})$, with $d \in \partial P_{d}(t, \cdot)$ on $\mathcal{T}$

Otherwise, $P_{d} \equiv \infty$ on all inputs.

Proof. If CMON is not satisfied, then there is a cycle $C=t_{0}, \ldots, t_{k}, t_{0}$ with positive sum. Then for any $t$ and $t^{\prime}$ the path $t C^{j} t^{\prime}$ that consists of starting at $t$, going to $t_{0}$, going around the cycle $j$ times, then going to $t^{\prime}$ has a sum that goes to infinity as $j$ goes to infinity. For the remainder, assume that CMON is satisfied.

(1) $P_{d}\left(t, t^{\prime}\right)+P_{d}\left(t^{\prime}, t^{\prime \prime}\right) \leq P_{d}\left(t, t^{\prime \prime}\right)$

$$
\begin{aligned}
& P_{d}\left(t, t^{\prime}\right)+P_{d}\left(t^{\prime}, t^{\prime \prime}\right) \\
& =\sup _{\substack{k \in \mathbb{N},\left\{t_{1}, \ldots, t_{k}\right\} \subseteq \mathcal{T} \\
t_{0}=t, t_{k+1}=t^{\prime}}} \sum_{i=0}^{k} d_{t_{i}}\left(t_{i+1}-t_{i}\right)+\sup _{\substack{k \in \mathbb{N},\left\{t_{1}, \ldots, t_{k}\right\} \subseteq \mathcal{T} \\
t_{0}=t^{\prime}, t_{k+1}=t^{\prime \prime}}} \sum_{i=0}^{k} d_{t_{i}}\left(t_{i+1}-t_{i}\right) \\
& =\sup _{\substack{j, k \in \mathbb{N},\left\{t_{1}, \ldots, t_{k}\right\} \subseteq \mathcal{T} \\
t_{0}=t, t_{j}=t^{\prime}, t_{k+1}=t^{\prime \prime}}} \sum_{i=0}^{k} d_{t_{i}}\left(t_{i+1}-t_{i}\right) \\
& \leq \sup _{\substack{k \in \mathbb{N},\left\{t_{1}, \ldots, t_{k}\right\} \subseteq \mathcal{T} \\
t_{0}=t, t_{k+1}=t^{\prime \prime}}} \sum_{i=0}^{k} d_{t_{i}}\left(t_{i+1}-t_{i}\right) \\
& =P_{d}\left(t, t^{\prime \prime}\right)
\end{aligned}
$$

(2) $d_{t}\left(t^{\prime \prime}-t\right) \leq P_{d}\left(t, t^{\prime \prime}\right)$

Taking $k=0$ shows that $d_{t}\left(t^{\prime \prime}-t\right)$ is an element of set over which the supremum is taken.

(3) $P_{d}(t, t)=0$

By CMON, $P_{d}(t, t) \leq 0$. By claim $(2), d_{t}(t-t)=0 \leq P_{d}(t, t)$.

(4) $P_{d}\left(t, t^{\prime}\right)+P_{d}\left(t^{\prime}, t\right) \leq 0$

By claims (1) and (3), $P_{d}\left(t, t^{\prime}\right)+P_{d}\left(t^{\prime}, t\right) \leq P(t, t)=0$.

(5) $P_{d}(t, \cdot)$ is convex and real-valued on $\operatorname{Conv}(\mathcal{T})$, with $d \in \partial P_{d}(t, \cdot)$ on $\mathcal{T}$

By CMON, for $t^{\prime} \in \mathcal{T} P_{d}\left(t, t^{\prime}\right) \leq-d_{t}\left(t_{0}-t^{\prime}\right)$. Thus, $P_{d}\left(t, t^{\prime}\right)$ is finite on $\mathcal{T}$. $P_{d}(t, \cdot)$ is a pointwise supremum of convex functions, so is convex. By convexity, it is also finite on 
$\operatorname{Conv}(\mathcal{T})$. For any $t^{\prime} \in \mathcal{T}$ and $t^{\prime \prime} \in \operatorname{Conv}(\mathcal{T})$

$$
\begin{aligned}
P_{d}\left(t, t^{\prime}\right)+d_{t^{\prime}}\left(t^{\prime \prime}-t^{\prime}\right) & =d_{t^{\prime}}\left(t^{\prime \prime}-t^{\prime}\right)+\sup _{\substack{k \in \mathbb{N},\left\{t_{1}, \ldots, t_{k}\right\} \subseteq \mathcal{T} \\
t_{0}=t, t_{k+1}=t^{\prime}}} \sum_{i=0}^{k} d_{t_{i}}\left(t_{i+1}-t_{i}\right) \\
& =\sup _{\substack{k \in \mathbb{N},\left\{t_{1}, \ldots, t_{k}\right\} \subseteq \mathcal{T} \\
t_{0}=t, t_{k}=t^{\prime} t_{k+1}=t^{\prime \prime}}} \sum_{i=0}^{k} d_{t_{i}}\left(t_{i+1}-t_{i}\right) \\
& \leq \sup _{\substack{k \in \mathbb{N},\left\{t_{1}, \ldots, t_{k}\right\} \subseteq \mathcal{T} \\
t_{0}=t, t_{k+1}=t^{\prime \prime}}} \sum_{i=0}^{k} d_{t_{i}}\left(t_{i+1}-t_{i}\right) \\
& =P\left(t, t^{\prime \prime}\right),
\end{aligned}
$$

so $d_{t}$ satisfies (2).

Having extracted the construction at the core of Rochet's proof, the rephrasing of his result as a statement about convex functions now follows easily.

Theorem B.3 (Adapted From Rochet 1987 ). A family $\left\{d_{t} \in \operatorname{Lin}(\mathcal{V} \rightarrow \mathbb{R})\right\}_{t \in \mathcal{T}}$ satisfies CMON if and only if there exists a convex $G: \operatorname{Conv}(\mathcal{T}) \rightarrow \mathbb{R}$ such that $\left\{d_{t}\right\}_{t \in \mathcal{T}} \in$ $\partial G$.

Proof. Given such a $G$, by 2 we have $d_{t_{i}}\left(t_{i+1}-t_{i}\right) \leq G\left(t_{i+1}\right)-G\left(t_{i}\right)$. Summing gives (28). Given such a family $\left\{d_{t}\right\}_{t \in \mathcal{T}}$, fix some $t_{0} \in \mathcal{T}$ and set $G: t \mapsto P_{d}\left(t_{0}, t\right)$. The result follows from Lemma B.2(5).

A number of papers have sought simpler and more natural conditions than CMON that are necessary and sufficient in special cases, e.g. Saks and Yu 2005; Archer and Kleinberg 2008: Ashlagi et al. 2010. These results are typically proven by showing they are equivalent to CMON. However, it is much more natural to directly construct the relevant $G$. As an example, we show one such result has a simple proof using our framework. This particular proof also has the advantage of providing a characterization of the payments that is more intuitive than the supremum in Rochet's construction.

As in Myerson's 1981 construction for the single-parameter case, we construct a $G$ by integrating over $d_{t}$. In particular, for any two types $x$ and $y$ our construction makes use of the line integral

$$
\int_{L_{x y}} d_{t}(y-x) d t=\int_{0}^{1} d_{(1-t) x+t y}(y-x) d t .
$$

As Berger et al. 2009 and Ashlagi et al. 2010 observed, if $\left\{d_{t}\right\}_{t \in \mathcal{T}}$ satisfies WMON and $\mathcal{T}$ is convex, this (Riemann) integral is well defined because it is the integral of a monotone function. If these line integrals vanish around all triangles (equivalently $\int_{L_{x y}} d_{t}(y-x) d t+$ $\left.\left.\int_{L_{y z}} d_{t}(z-y) d t=\int_{L_{x z}} d_{t}(z-x) d t\right)\right)$ we say $\left\{d_{t}\right\}$ satisfies path independence.

TheOrem B.4 (ADAPTED From [MüLLER ET Al. 2007]). For convex $\mathcal{T}$, a family $\left\{d_{t} \in \operatorname{Lin}(\mathcal{V} \rightarrow \mathbb{R})\right\}_{t \in \mathcal{T}}$ is a selection of subgradients of a convex function if and only if $\left\{d_{t}\right\}_{t \in \mathcal{T}}$ satisfies WMON and path independence.

Proof. Given a convex function $G$ and selection of subgradients $\left\{d_{t}\right\},\left\{d_{t}\right\}$ satisfies CMON and thus WMON. Path independence also follows from convexity (Rockafellar 1997] p. 232). Now given a $\left\{d_{t}\right\}$ that satisfies WMON and path independence, fix a type $t_{0} \in \mathcal{T}$ 
and define $G\left(t^{\prime}\right)=\int_{L_{t_{0} t^{\prime}}} d_{t}\left(t^{\prime}-t_{0}\right) d t$ (well defined by WMON as the integral of a monotone function). Given $x, y, z \in \mathcal{T}$ such that $z=\lambda x+(1-\lambda) y$, by path independence and the linearity of $d_{z}$ we have

$$
\begin{aligned}
& \lambda G(x)+(1-\lambda) G(y) \\
& =G(z)+\lambda \int_{L_{z x}} d_{t}(x-z) d t+(1-\lambda) \int_{L_{z y}} d_{t}(y-z) d t \\
& \geq G(z)+\lambda d_{z}(x-z)+(1-\lambda) d_{z}(y-z)=G(z),
\end{aligned}
$$

so $G$ is convex. Similarly, for $x, y \in \mathcal{T}, d_{t}$ satisfies $(2)$ because

$$
d_{x}(y-x) \leq \int_{L_{x y}} d_{t}(y-x) d t=G(y)-G(x) .
$$

\section{B.2. Local Characterizations}

In many settings, it is easier to reason about the behavior of a mechanism given small changes to its input rather than arbitrary changes, so several authors have sought to characterize truthful mechanisms using local conditions Archer and Kleinberg 2008, Berger et al. 2009: Carroll 2012. We show in this section how many of these results are in essence a consequence of a more fundamental statement, that convexity is an inherently local property. For example, in the twice differentiable case it can be verified by determining whether the Hessian is positive semidefinite at each point. We start with a local convexity result, and use it to show that an affine score is truthful if and only if it satisfies a very weak local truthfulness property introduced by Carroll 2012. Afterwards we turn to a characterization by Archer and Kleinberg 2008 that proved a similar theorem for a different notion of local truthfulness. Our results (specifically Theorem B.6 show that these two notions of local truthfulness are equivalent because Archer and Kleinberg's definition corresponds to the property of being a local subgradient, while Carroll's corresponds to the property of being a weak, local subgradient, which we now define.

Definition B.5. Let $\mathcal{T}$ be convex. A family $\left\{d_{t} \in \operatorname{Lin}(\mathcal{V} \rightarrow \overline{\mathbb{R}})\right\}_{t \in \mathcal{T}}$ is a weak local subgradient $(W L S G$ ) of a convex function $G: \mathcal{T} \rightarrow \overline{\mathbb{R}}$ if for all $t \in \mathcal{T}$ there exists an open neighborhood $U_{t}$ of $t$ such that for all $t^{\prime} \in U_{t}$,

$$
G(t) \geq G\left(t^{\prime}\right)+d_{t^{\prime}}\left(t-t^{\prime}\right) \quad \text { and } \quad G\left(t^{\prime}\right) \geq G(t)+d_{t}\left(t^{\prime}-t\right) .
$$

Furthermore, if for every $s \in \mathcal{T}$, eq. (31) holds for all $t, t^{\prime} \in U_{s}$, we say $\left\{d_{t}\right\}_{t \in \mathcal{T}}$ is a local subgradient ( $L S G)$ of $G$.

We now show that being a WLSG is a necessary and sufficient condition for a family of functions to be a selection of subgradients. The proof is heavily inspired by Carroll 2012 .

Theorem B.6. Let $\mathcal{T}$ be convex. A family $\left\{d_{t} \in \operatorname{Lin}(\mathcal{V} \rightarrow \overline{\mathbb{R}})\right\}_{t \in \mathcal{T}}$ is a selection of subgradients of a convex function $G: \mathcal{T} \rightarrow \overline{\mathbb{R}}$ if and only if it is a WLSG of $G$.

Proof (Adapted from Carrol 2012|). As usual, the forward direction is trivial. For the other, let $t, t^{\prime} \in \mathcal{T}$ be given; we show that the subgradient inequality for $d_{t^{\prime}}$ holds at $t$. By compactness of $\operatorname{Conv}\left(\left\{t, t^{\prime}\right\}\right)$, we have a finite set $t_{i}=\alpha_{i} t^{\prime}+\left(1-\alpha_{i}\right) t$, where $0=\alpha_{0} \leq \cdots \leq \alpha_{k+1}=1$, such that WLSG holds between each $t_{i}$ and $t_{i+1}$. (The cover $\left\{U_{s} \mid s \in \operatorname{Conv}\left(\left\{t, t^{\prime}\right\}\right)\right\}$ has a finite subcover. Take $t_{2 j}$ from the subcover and $t_{2 j+1} \in U_{t_{2 j}} \cap$ $U_{t_{2 j+2}}$.) By the WLSG condition (31), we have for each $i$,

$$
\begin{aligned}
& 0 \geq G\left(t_{i+1}\right)-G\left(t_{i}\right)+d_{t_{i+1}}\left(t_{i}-t_{i+1}\right) \\
& 0 \geq G\left(t_{i}\right)-G\left(t_{i+1}\right)+d_{t_{i}}\left(t_{i+1}-t_{i}\right) .
\end{aligned}
$$


Now using the identity $t_{i+1}-t_{i}=\left(\alpha_{i+1}-\alpha_{i}\right)\left(t^{\prime}-t\right)$ and adding $\alpha_{i} /\left(\alpha_{i+1}-\alpha_{i}\right)$ times 32 to $\alpha_{i+1} /\left(\alpha_{i+1}-\alpha_{i}\right)$ times $(33)$, we have

$$
0 \geq G\left(t_{i}\right)-G\left(t_{i+1}\right)+\alpha_{i+1} d_{t_{i}}\left(t^{\prime}-t\right)-\alpha_{i} d_{t_{i+1}}\left(t^{\prime}-t\right) .
$$

Summing (34) over $0 \leq i \leq k$ gives

$$
0 \geq G\left(t_{0}\right)-G\left(t_{k+1}\right)+\alpha_{k+1} d_{t_{0}}\left(t^{\prime}-t\right)-\alpha_{0} d_{t_{k+1}}\left(t^{\prime}-t\right),
$$

which when recalling our definitions for $\alpha_{i}$ and $t_{i}$ yields the result.

The WLSG condition translates to an analogous notion in terms of truthfulness, weak local truthfulness.

Definition B.7. An affine score is weakly locally truthful if for all $t \in \mathcal{T}$ there exists some open neighborhood $U_{t}$ of $t$, such that truthfulness holds between $t$ and every $t^{\prime} \in U_{t}$, and vice versa. That is,

$$
\forall t \in \mathcal{T}, \forall t^{\prime} \in U_{t}, \quad \mathrm{~A}\left(t^{\prime}, t\right) \leq \mathrm{A}(t, t) \text { and } \mathrm{A}\left(t, t^{\prime}\right) \leq \mathrm{A}\left(t^{\prime}, t^{\prime}\right) .
$$

Corollary B.8 (Generalization of Carroll 2012 ). An affine score A : $\mathcal{T} \times$ $\mathcal{T} \rightarrow \overline{\mathbb{R}}$ for convex $\mathcal{T}$ is truthful if and only if it is weakly locally truthful.

Proof. Defining $G(t):=\mathrm{A}(t, t)$, by weak local truthfulness we may write

$$
\begin{aligned}
G(t)=\mathrm{A}(t, t) & \geq \mathrm{A}\left(t^{\prime}, t\right)=G\left(t^{\prime}\right)+\mathrm{A}_{\ell}\left(t^{\prime}, t-t^{\prime}\right) \\
G\left(t^{\prime}\right)=\mathrm{A}\left(t^{\prime}, t^{\prime}\right) & \geq \mathrm{A}\left(t, t^{\prime}\right)=G(t)+\mathrm{A}_{\ell}\left(t, t^{\prime}-t\right),
\end{aligned}
$$

where $t^{\prime}$ is local to $t$ and $\mathrm{A}_{\ell}(t, \cdot)$ is the linear part of $\mathrm{A}(t, \cdot)$. This says that $d_{t}=\mathrm{A}_{\ell}(t, \cdot)$ satisfies WLSG for convex function $G$; the rest follows from Theorem B.6 and Theorem 2.3.

Finally, in the spirit of Section B.1. Archer and Kleinberg 2008 characterized local conditions under which an allocation rule can be made truthful. A key condition from their paper is vortex-freeness, which is a condition they show to be equivalent to local path independence (analagous to our terminology of weak local subgradients it can be thought of as weak local path independence). The other condition, local WMON, means that WMON holds in some neighborhood around each type. Their result then follows from the observation that local WMON and local path independence imply local subgradient. While this particular proof is not significantly simpler than the original, we believe it is somewhat more natural and clarifies the connection between the underlying reasons a notion of local truthfulness suffices both here and in Carroll's setting.

Corollary B.9. Let $\mathcal{T}$ be convex. A family $\left\{d_{t} \in \operatorname{Lin}(\mathcal{V} \rightarrow \mathbb{R})\right\}_{t \in \mathcal{T}}$ is a selection of subgradients of a convex function if and only if it satisfies local WMON and is vortex-free.

Proof.

We prove the reverse direction; suppose $\left\{d_{t}\right\}_{t \in \mathcal{T}}$ satisfies local WMON and is vortex-free. From Lemma 3.5 of Archer and Kleinberg 2008] we have that vortex-freeness is equivalent to path independence, so by Theorem B.4 for all $t$ there exists some open $U_{t}$ such that $\left\{d_{t^{\prime}}\right\}_{t^{\prime} \in U_{t}}$ is the subgradient of some convex function $G^{(t)}: U_{t} \rightarrow \mathbb{R}$. We need only show the existence of some $G$ such that $\left\{d_{t}\right\}_{t \in \mathcal{T}}$ is the subgradient of $G$ on each $U_{t}$; the rest follows from Theorem B.6.

Fix some $t_{0} \in \mathcal{T}$ and define $G(t)=\int_{L_{t_{0} t}} d_{t^{\prime}} d t^{\prime}$, which is well defined by compactness of $\operatorname{Conv}\left(\left\{t_{0}, t\right\}\right)$ and the fact that a locally increasing real-valued function is increasing. But for each $t^{\prime}$ and $t \in U_{t^{\prime}}$ we can also write $G^{\left(t^{\prime}\right)}(t)=\int_{L_{t^{\prime} t} t} d_{t^{\prime \prime}} d t^{\prime \prime}$ by Rockafellar 1997, p. 232], and now by path independence we see that $G$ and $G^{\left(t^{\prime}\right)}$ differ by a constant. Hence $\left\{d_{t}\right\}_{t \in \mathcal{T}}$ must be a subgradient of $G$ on $U_{t^{\prime}}$ as well, for all $t^{\prime} \in \mathcal{T}$. 


\section{REVENUE EQUIVALENCE}

Perhaps the most celebrated result in auction theory is the revenue equivalence theorem, which states that, in a single item auction, the revenue from an agent (equivalently that agent's consumer surplus) is determined up to a constant by the equilibrium probability that each possible type of that agent will receive the item [Myerson 1981]. A large body of work has looked for more general conditions under which this property holds (see, e.g., Krishna and Maenner 2001]) or what can be said when it does not Carbajal and Ely 2012. One general approach is due to Heydenreich et al. 2009, who use a graphical representation related to CMON. Given our main theorem, this is unsurprising. In convex analysis terms, asking whether an implementable allocation rule satisfies revenue equivalence is asking whether all convex functions that have a selection of their subgradients that corresponds to that allocation rule are the same up to a constant. As we saw in the proof of Lemma B.2, CMON permits the natural construction of a convex function from its subgradient via (28). Intuitively, if we know the payments we want for some subset of types, we can check if those are consistent with a desired payment for some other type by checking whether this construction still works, both in terms of the constraints of the existing types on the new one and the new one on the existing ones. The following theorem applies this insight to get a result that is stronger than revenue equivalence as iteratively applying it characterizes the possible payments for every mechanism.

Theorem C.1. Let $G$ be a convex function on $\operatorname{Conv}(\mathcal{T}), d=\left\{d_{t}\right\}_{t \in \mathcal{T}}$ a selection of its subgradients on $\mathcal{T}, S \subseteq \mathcal{T}$ non-empty, $t^{*} \in \mathcal{T} \backslash S$, and c be given. Then there exists a convex $G^{\prime}$ on $\operatorname{Conv}(\mathcal{T})$ agreeing with $G$ on $S$, with $\left\{d_{t}\right\}_{t \in \mathcal{T}} \in \partial G^{\prime}$ and $G^{\prime}\left(t^{*}\right)=c$, if and only if

$$
\sup _{t_{0} \in S} G\left(t_{0}\right)+P_{d}\left(t_{0}, t^{*}\right) \leq c \leq \inf _{t_{0} \in S} G\left(t_{0}\right)-P_{d}\left(t^{*}, t_{0}\right)
$$

Proof. Given such a $G^{\prime}$, the LHS of (36) becomes $\sup _{t_{0} \in S} G^{\prime}\left(t_{0}\right)+P_{d}\left(t_{0}, t^{*}\right) \leq G^{\prime}\left(t^{*}\right)$. Applying the definition of $P_{d}(29)$ and then repeatedly applying the subgradient inequality (2) yields the desired inequality. Similarly, the RHS of (36) can be rewritten as $G^{\prime}\left(t^{*}\right)+P_{d}\left(t^{*}, t_{0}\right) \leq G^{\prime}\left(t_{0}\right)$ for all $t_{0} \in S$, and the definition and subgradient inequality applied.

Now suppose (36) holds. Let $G^{\prime}(t) \doteq \max \left\{c+P_{d}\left(t^{*}, t\right), \sup _{t_{0} \in S} G\left(t_{0}\right)+P_{d}\left(t_{0}, t\right)\right\}$. By Theorem B.3, $d$ satisfies CMON, so by Lemma B.2 $G^{\prime}$ is convex, finite-valued on $\operatorname{Conv}(\mathcal{T})$, and has $\left\{d_{t}\right\} \in \partial G^{\prime}$. Hence, we need only show that $G^{\prime}$ agrees with $G$ on $S$ and has $G^{\prime}\left(t^{*}\right)=c$.

First, fixing any $t \in S$, we will establish the following:

$$
G(t)=\sup _{t_{0} \in S} G\left(t_{0}\right)+P_{d}\left(t_{0}, t\right) .
$$

As $P_{d}(t, t)=0$ from Lemma B.2(3), we have $G(t)=G(t)+P_{d}(t, t) \leq \sup _{t_{0} \in S} G\left(t_{0}\right)+$ $P_{d}\left(t_{0}, t\right)$. Furthermore, $G\left(t_{0}\right)+P_{d}\left(t_{0}, t\right) \leq G(t)$ for all $t_{0} \in \mathcal{T}$ by repeated application of the subgradient inequality (2). Hence, we have $\sup _{t_{0} \in S} G\left(t_{0}\right)+P_{d}\left(t_{0}, t\right) \leq G(t)$ as well.

By eq. (37), we can write $G^{\prime}(t)=\max \left\{c+P_{d}\left(t^{*}, t\right), G(t)\right\}$ when $t \in S$. But by the RHS of eq. (36), we see $c+P_{d}\left(t^{*}, t\right) \leq G(t)$, so $G^{\prime}(t)=G(t)$. Similarly, applying the LHS of eq. (36) and $P_{d}\left(t^{*}, t^{*}\right)=0$ to the definition of $G^{\prime}\left(t^{*}\right)$, we have $G^{\prime}\left(t^{*}\right)=c$.

Viewed through Theorem C.1 revenue equivalence holds when the upper and lower bounds from (36) match after the value of $G$ is fixed at a single point. This allows us to derive a necessary and sufficient condition for revenue equivalence that is equivalent to that given by Heydenreich et al. 2009 and actually applies to all affine scores. For example, this gives a revenue equivalence theorem for mechanisms with partial allocation.

Corollary C.2 (Revenue Equivalence). Let a truthful affine score A : $\mathcal{T} \times \mathcal{T} \rightarrow \overline{\mathbb{R}}$ be given, and $d=\left\{d_{t}\right\}_{t \in \mathcal{T}}$ be the corresponding selection of subgradients from (3). Then ev- 
ery truthful affine score $\mathrm{A}^{\prime}: \mathcal{T} \times \mathcal{T} \rightarrow \overline{\mathbb{R}}$ with the same corresponding selection of subgradients differs from $\mathrm{A}$ by a constant (i.e. $\mathrm{A}\left(t^{\prime}, t\right)=\mathrm{A}^{\prime}\left(t^{\prime}, t\right)+c$ ) if and only if $P_{d}\left(t^{\prime}, t\right)+P_{d}\left(t, t^{\prime}\right)=0$ for all $t, t^{\prime} \in \mathcal{T}$.

Proof. We will show that the convex function $G$ from eq. (3) is unique up to a constant if and only if $P_{d}\left(t^{\prime}, t\right)+P_{d}\left(t, t^{\prime}\right)=0$ for all $t, t^{\prime} \in \mathcal{T}$.

For the forward direction, let $t_{0} \in \mathcal{T}$ be arbitrary. Then for all $t \in \mathcal{T}$, taking (36) with $S=$ $\left\{t_{0}\right\}$ and $G(t) \doteq c+P_{d}\left(t_{0}, t\right)$ gives the condition $G\left(t_{0}\right)+P_{d}\left(t_{0}, t\right) \leq G^{\prime}(t) \leq G\left(t_{0}\right)-P_{d}\left(t, t_{0}\right)$ for the value of $G^{\prime}(t)$. But as $P_{d}\left(t, t_{0}\right)=-P_{d}\left(t_{0}, t\right)$ we have $G^{\prime}(t)=P_{d}\left(t_{0}, t\right)+G\left(t_{0}\right)=G(t)$ for all $t$.

For the reverse direction, assume $P_{d}\left(t^{1}, t^{2}\right) \neq-P_{d}\left(t^{2}, t^{1}\right)$ for some $t^{1}, t^{2} \in \mathcal{T}$, and let $G^{1}(t) \doteq P_{d}\left(t^{1}, t\right)$ and $G^{2}(t) \doteq P_{d}\left(t^{1}, t^{2}\right)+P_{d}\left(t^{2}, t\right)$. We easily check from Lemma B.2 (3) that $G^{1}\left(t^{2}\right)=G^{2}\left(t^{2}\right)=P_{d}\left(t^{1}, t^{2}\right)$, but we have $G^{1}\left(t^{1}\right)=0$ while $G^{2}\left(t^{1}\right)=P_{d}\left(t^{1}, t^{2}\right)+P_{d}\left(t^{2}, t^{1}\right) \neq$ 0 .

We note that these two results are similar to results of Kos and Messner 2012. The main novelties in our version are showing that every value in the interval yields a convex function (as opposed to merely the extremal ones), the ability to characterize possible values after the values at multiple points are fixed (as opposed to a single point), and the framing in terms of convex analysis.

The conditions given by Theorem C.1 and Corollary C.2, while general, are not particularly intuitive. However, there are a number of special cases where they do have natural interpretations for mechanism design. The first is when the set of types is finite. In this setting (explored in an auction theory context in, e.g., Diakonikolas et al. 2012]) it is well known that revenue equivalence does not hold. The finite set of constraints (36) can be used in general as a linear program to, e.g., maximize revenue (see Section 6.5.2 of Vohra 2011 for an example). In particular cases, they may become simple enough to have a nice characterization. For example, in the single-parameter setting only a linear number of paths need be considered. This setting is illustrated in Figure 4.

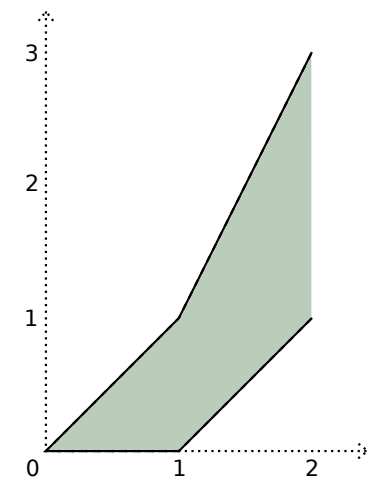

(a)

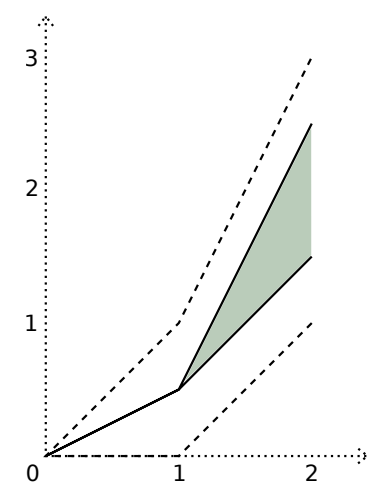

(b)

Fig. 4: Consider a one-dimensional setting with type space $\mathcal{T}=\{0,1,2\}$ and $d_{0}=0, d_{1}=$ $1, d_{2}=2$. In (a), we fix $G(0)=0$, yielding a range of possible values dictated by the subgradients: $0 \leq G(1) \leq 1$ and $1 \leq G(2) \leq 3$. We can pick any point in the resulting set and fix $\mathrm{G}$ there. However, we cannot pick any increasing function: in (b), we fix $G(1)=0.5$, restricting $G(2)$ to the interval $[1.5,2.5]$.

More broadly, as we saw in the proof of Theorem B.4 the (supremum over the) sum can often be interpreted as an integral. In particular, the fact that $G$ is convex guarantees that 
(under mild conditions) integrals of a selection of its subgradient are path independent and the integral from $t$ to $t^{\prime}$ gives $G\left(t^{\prime}\right)-G(t)$. If $\mathcal{T}$ is connected by smooth paths (e.g. if it is convex), this means that $\mathcal{T}$ satisfies revenue equivalence for all implementable mechanisms (previously shown under a somewhat different notion of the set of types Heydenreich et al. 2009]). As it is particularly simple to prove, we state the version for convex $\mathcal{T}$.

COROllary C.3. Let $\mathcal{T}$ be convex, a truthful affine score $A: \mathcal{T} \times \mathcal{T} \rightarrow \overline{\mathbb{R}}$ be given, and $\left\{d G_{t}\right\}_{t \in \mathcal{T}}$ be the corresponding selection of subgradients from (3). Then any truthful affine score $\mathrm{A}^{\prime}: \mathcal{T} \times \mathcal{T} \rightarrow \overline{\mathbb{R}}$ with the same corresponding selection of subgradients differs from $\mathrm{A}$ by a constant (i.e. $\left.\mathrm{A}\left(t^{\prime}, t\right)=\mathrm{A}^{\prime}\left(t^{\prime}, t\right)+c\right)$.

Proof. By Theorem 2.3, we know that $A$ and $A^{\prime}$ only differ only in their choice of convex function $G$. However, each choice has the same selection of subgradients, and two convex functions with the same selection of subgradents differ by a constant Rockafellar 1997. For intuition, see the construction of $G$ by integrating its subgradients in the proof of Theorem B.4.

\section{DUALITY IN ELICITIATION}

We now provide mathematical foundation for notions of duality from $\S 5$. To begin, we need a dual vector space with more structure than $\operatorname{simply} \operatorname{Lin}(\mathcal{V} \rightarrow \mathbb{R})$. For this we use the notion of a dual pair, which is a standard setting for convex analysis in infinite-dimensional spaces.

Definition D.1 ( Aliprantis and Border 2007, § 5.14]). A pair of topological vector spaces $\left(\mathcal{V}, \mathcal{V}^{*}\right)$ is a dual pair if it is equipped with a bilinear form $\langle\cdot, \cdot\rangle: \mathcal{V} \times \mathcal{V}^{*} \rightarrow \mathbb{R}$ which separates points, in the sense that $\forall v^{*}\langle v, \cdot\rangle \equiv 0$ implies $v=0$ and $\forall v\left\langle\cdot, v^{*}\right\rangle \equiv 0$ implies $v^{*}=0$.

Note that the above assumption that $\left(\mathcal{V}, \mathcal{V}^{*}\right)$ is a dual pair implies in particular that for all $v^{*} \in \mathcal{V}^{*}$, the map $v^{*} \mapsto\left\langle v, v^{*}\right\rangle$ is linear. This is crucial when interpreting $\mathcal{R} \subseteq \mathcal{V}^{*}$ as the type space, since affine scores must be affine in the type. For the remainder of this section we assume that we have a dual pair $\left(\mathcal{V}, \mathcal{V}^{*}\right)$.

A natural question is to determine the conditions under which we have $G^{* *} \doteq\left(G^{*}\right)^{*}=G$. That is, when is the conjugacy operator an involution? This has been thoroughly studied in convex analysis. We state the classic theorem due to Fenchel and Moreau Ioffe and Tikhomirov 1979, Lai and Lin 1988.

Definition D.2. A function $f: X \rightarrow \overline{\mathbb{R}}$ is lower semi-continuous (l.s.c.) if for every $x_{0}$ in $\operatorname{dom}(f)$ it holds that $\liminf _{x \rightarrow x_{0}} f(x) \geq f\left(x_{0}\right)$.

TheOREM D.3 (FEnCHEL-MOREAU). Let $X$ be a Hausdorff locally convex space. For any function $G: X \rightarrow \overline{\mathbb{R}}$, it follows that $G=G^{* *}$ if and only if one of the following is true: (1) $G$ is a proper, l.s.c., and convex function, (2) $G \equiv+\infty$, or (3) $G \equiv-\infty$.

The following corollary will prove very helpful in our discussion of type duality below. The proof follows from applying Theorem D.3 (note that as $\mathbb{R}$ is Hausdorff, $\mathcal{V}$ together with the product topology inherited from the dual pair is also Hausdorff and locally convex; see [Aliprantis and Border 2007, § 7] for details), and then Lemma 4 twice, once for $G$ and once for $G^{*}$.

Corollary D.4. If $G$ is convex, proper, and l.s.c., then $v^{*} \in \partial G_{v} \Longleftrightarrow v \in \partial G_{v^{*}}^{*}$.

We now introduce the concept of a dual property $\Gamma^{*}$, which essentially swaps the type and the report. That is, an agent has a "true report" $r$ and $\Gamma^{*}(r)$ encodes all the "correct types" $t$. We then go on to show the relationship between the subgradient elicitability of dual properties. 


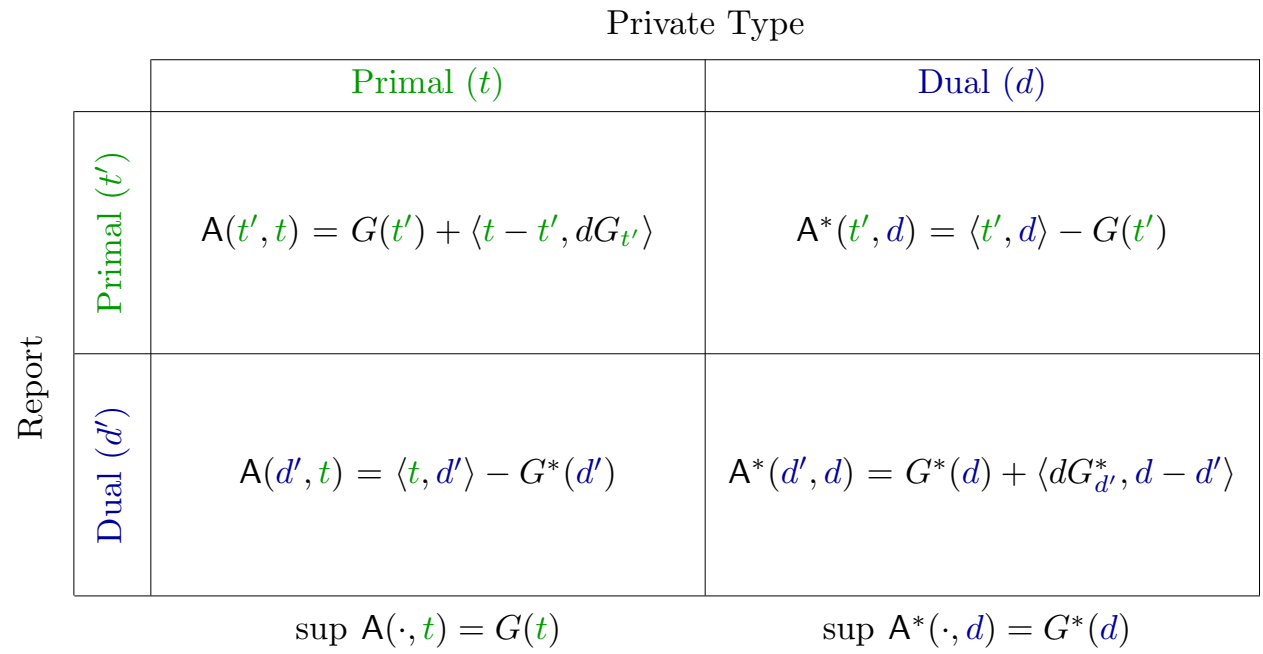

Table II: The general duality quadrangle.

Definition D.5. Let $\Gamma: \mathcal{T} \rightrightarrows \mathcal{R}$ where $\mathcal{R} \subseteq \mathcal{V}^{*}$. Then the dual of $\Gamma$, written $\Gamma^{*}: \mathcal{R} \rightrightarrows \mathcal{T}$, is defined by $\Gamma^{*} \doteq \Gamma^{-1}$. In other words, $\Gamma^{*}$ satisfies $r \in \Gamma(t) \Longleftrightarrow t \in \Gamma^{*}(r)$.

TheOREM D.6. For dual pair $\left(\mathcal{V}, \mathcal{V}^{*}\right)$, let $\Gamma: \mathcal{T} \rightrightarrows \mathcal{D}$ be given with $\mathcal{T} \subseteq \mathcal{V}$ and $\mathcal{D} \subseteq \mathcal{V}^{*}$ Let convex proper and l.s.c. $G$ be given. Then $G$ elicits $\Gamma$ if and only if $G^{*}$ elicits $\Gamma^{*}$.

Proof. We apply Corollary D.4 to obtain $d \in \partial G_{t} \Longleftrightarrow t \in \partial G_{d}^{*}$. If $G$ subgradientelicits $\Gamma$, then we have

$$
t \in \Gamma^{*}(d) \Longleftrightarrow d \in \Gamma(t) \Longleftrightarrow d \in \partial G_{t} \Longleftrightarrow t \in \partial G_{d}^{*},
$$

so $G^{*}$ subgradient-elicits $\Gamma^{*}$. Clearly the above may be applied in the reverse direction as well, yielding the result.

Note that when $G$ and $G^{*}$ elicit $\Gamma$ and $\Gamma^{*}$, respectively, we have by the above discussion that $\mathrm{A}(d, t)=\langle t, d\rangle-G^{*}(d)$ elicits $\Gamma$ and $\mathrm{A}^{*}(t, d)=\langle t, d\rangle-G(t)$ elicits $\Gamma^{*}$. Moreover, the "consumer surplus" functions of A and $\mathrm{A}^{*}$ are $G$ and $G^{*}$, respectively. This curious relationship, combined with the notion of report duality, can be visualized as shown in Table II] the general version of Table II. Note that traveling around the table does not necessarily mean arriving at the same choice of $G$, nor does it imply that $G^{* *}=G$. However, when $G^{* *}=G$ does hold, the diagram "commutes" in a certain sense.

We conclude with a few remarks.

Identities. Table I shows that elicitation inherits a lot of structure from convex duality. Ignoring boundary and regularity concerns for the moment, and looking at general types $t$, we obtain some nice identities:

$$
\begin{aligned}
& \mathrm{A}(d, t)+\mathrm{A}^{*}(t, d) \geq\langle t, d\rangle \\
& \mathrm{A}(d, t)-\mathrm{A}^{*}(t, d)=G(t)-G^{*}(d) .
\end{aligned}
$$

The first follows from the classic Fenchel-Young inequality [Rockafellar 1997, the proof of which for $G$ proper follows directly from the definition of the conjugate (Definition 5.4).

Lemma D.7 (Fenchel-Young inequality). $\forall v \in \mathcal{V}, v^{*} \in \mathcal{V}^{*}, G(v)+G^{*}\left(v^{*}\right) \geq$ $\left\langle v, v^{*}\right\rangle$. 
Score divergences. The score divergence $\mathrm{A}(t, t)-\mathrm{A}\left(t^{\prime}, t\right)$ is a natural notion of "regret" which arises frequently in the scoring rules literature (cf. Gneiting and Raftery 2007]). Our score divergence, as we define below, is reminiscent of a Bregman divergence.

$$
D_{G, d G}\left(t, t^{\prime}\right) \doteq \mathrm{A}(t, t)-\mathrm{A}\left(t^{\prime}, t\right)=G(t)-G\left(t^{\prime}\right)-\left\langle t-t^{\prime}, d G_{t^{\prime}}\right\rangle .
$$

Note that the first argument to $D$ is the true type, as opposed to our A notation. Also note the subscripts to $D$, which specify both the convex function $G$ and a selection of subgradients. A Bregman divergence requires $G$ to be continuously differentiable, but our definition (40) is a natural extension, and has been studied before (cf. Iyer and Bilmes 2013 ).

Score divergences have many nice properties, like convexity in the first argument, and (directional) differentiability at $t^{\prime}=t$. Score divergences also enable reasoning about the magnitude of off-equilibrium payoffs, which can be important in practice, when externalities are often present. For example, Fiat et al. 2013] introduce the notion of "strong truthfulness", where the payoff decays as $\left\|t-t^{\prime}\right\|^{2}$, to design mechanisms that are robust even when agents care about the utility of other agents.

Turning to our various notions of duality, the following are four divergences corresponding to the duality quadrangle, starting in the (primal,primal) setting and moving counterclockwise.

$$
\begin{aligned}
D_{G, d G}\left(t, t^{\prime}\right) & =G(t)-G\left(t^{\prime}\right)-\left\langle t-t^{\prime}, d G_{t^{\prime}}\right\rangle \\
D_{G}\left(t, d^{\prime}\right) & =G(t)+G^{*}\left(d^{\prime}\right)-\left\langle t, d^{\prime}\right\rangle \\
D_{G^{*}, d G^{*}}\left(d, d^{\prime}\right) & =G^{*}(d)-G^{*}\left(d^{\prime}\right)-\left\langle d G_{d^{\prime}}^{*}, d-d^{\prime}\right\rangle \\
D_{G^{*}}\left(d, t^{\prime}\right) & =G^{*}(d)+G\left(t^{\prime}\right)-\left\langle t^{\prime}, d\right\rangle .
\end{aligned}
$$

Interestingly, we see that $D_{G}(t, d)=D_{G^{*}}(d, t)$ for all $t, d$ (not just dual points). In other words, the loss of reporting $d$ in the primal but having type $t$ is the same as reporting $t$ in the dual but having "type" $d$.

\section{E. BREGMAN VORONOI DIGRAMS AND THE ROLE OF $\|\cdot\|^{2}$}

The squared norm seems fundamental to our derivation in $\S 6$, let us dig further to see if this is indeed the case. Observe that the form 24) is simply

$$
\mathrm{A}(r, t)=2\left\langle t_{r}, t\right\rangle-\left\|t_{r}\right\|^{2}+w_{r},
$$

where $t_{r}=\alpha p_{r}+p_{0}$. Consider the case where $w_{r}=0$ for all $r$, which corresponds to $\Gamma$ being a Voronoi diagram. In this case, could think of $\mathrm{A}$ as being a special case of the "Brier score" $\mathrm{A}^{B}\left(t^{\prime}, t\right)=2\left\langle t,^{\prime} t\right\rangle-\left\|t^{\prime}\right\|^{2}$, so that $\mathrm{A}(r, t)=\mathrm{A}^{B}\left(t_{r}, t\right)$. In other words, we can think of our finite-report case as just restricting the allowed reports in a general direct-revelation affine score. Note that the score divergence for $\mathrm{A}^{B}$ is just $D_{G}\left(t^{\prime}, t\right)=\left\|t^{\prime}-t\right\|^{2}$, where $G(t)=\|t\|^{2}$ is just the square norm. This raises the following interesting question: what do we get when we replace $G=\|\cdot\|^{2}$ with another convex function on $\mathcal{T}$, and restrict the reports from $\mathcal{T}$ to just a few points $\left\{t_{r}\right\}_{\mathcal{R}}$ ? That is, take $\mathrm{A}^{G}\left(t^{\prime}, t\right)=G\left(t^{\prime}\right)-d G_{t^{\prime}}\left(t-t^{\prime}\right)$ and set $\mathrm{A}(r, t)=\mathrm{A}^{G}\left(t_{r}, t\right)$. Surely, for any such $G$, whatever $\Gamma$ is elicited by such a modified affine score would have to be a diagram by Theorem 6.2 . But then why does the squared norm seem so fundamental?

As it happens, we are touching on precisely the notion of a Bregman Voronoi diagram, introduced by Boissonnat et al. 2007, $\S$ 4]. There, instead of defining cell $_{i}=\{t: i \in$ $\left.\operatorname{argmin}_{j}\left\|t_{j}-t\right\|\right\}$, the squared norm is replaced by any Bregman divergence $D_{G}$, so that $\operatorname{cell}_{i}=\left\{t: i \in \operatorname{argmin}_{j} D_{G}\left(t, t_{j}\right)\right\}{ }^{10}$ Our conclusion that such diagrams coincide with power diagrams corresponds to their Theorem 8 .

\footnotetext{
${ }^{10}$ In Boissonnat et al. 2007, three types of diagrams are introduced; here we refer to the first type.
} 
Framed in terms of our report duality from $\S 5.2$, we can see this yet another way. We can rewrite the Bregman Voronoi cell as

$$
\operatorname{cell}_{i}=\left\{t: i \in \underset{j}{\operatorname{argmax}} G\left(t_{j}\right)+d G_{t_{j}}\left(t-t_{j}\right)\right\} .
$$

By Lemma 4, this can in turn be written

$$
\operatorname{cell}_{i}=\left\{t: i \in \underset{j}{\operatorname{argmax}}\left\langle\tilde{t}_{j}, t\right\rangle-G^{*}\left(\tilde{t}_{j}\right)\right\}
$$

where $\tilde{t}_{j}=d G_{t_{j}}$. Hence, for any convex function $G$, the sites $\left\{p_{j}\right\}$ and weights $w$ of a power diagram corresponding to the $D_{G}$ Bregman Voronoi diagram with sites $\left\{t_{j}\right\}$ are given by $p_{j}=\frac{1}{2} d G_{t_{j}}$ and $w_{j}=\frac{1}{4}\left\|d G_{t_{j}}\right\|^{2}-G^{*}\left(d G_{t_{j}}\right)$.

\section{Acknowledgments}

We would like to thank Aaron Archer, Gabriel Carroll, Yiling Chen, Philip Dawid, Felix Fischer, Hu Fu, Philippe Jehiel, Nicolas Lambert, Peter Key, David Parkes, Colin Rowat, Greg Stoddard, Matus Telgarsky, and Rakesh Vohra for helpful discussions, and anonymous reviewers for helpful feedback. This work was supported in part by National Science Foundation Grant CCF-1657598.

\section{REFERENCES}

Abernethy, J., Chen, Y., And Vaughan, J. W. 2013. Efficient market making via convex optimization, and a connection to online learning. ACM Transactions on Economics and Computation 1, $2,12$.

Abernethy, J. And Frongillo, R. 2012. A characterization of scoring rules for linear properties. In Proceedings of the 25th Conference on Learning Theory. 1-27.

Aliprantis, C. D. And Border, K. C. 2007. Infinite Dimensional Analysis: A Hitchhiker's Guide. Springer.

Archer, A. And Kleinberg, R. 2008. Truthful germs are contagious: a local to global characterization of truthfulness. In Proceedings of the 9th ACM Conference on Electronic Commerce. 21-30.

Ashlagi, I., Braverman, M., Hassidim, A., And Monderer, D. 2010. Monotonicity and implementability. Econometrica 78, 5, 1749-1772.

Aurenhammer, F. 1987a. A criterion for the affine equivalence of cell complexes in R d and convex polyhedra in R d+1. Discrete \& Computational Geometry 2, 1, 49-64.

Aurenhammer, F. 1987b. Power diagrams: properties, algorithms and applications. SIAM Journal on Computing 16, 1, 78-96.

Aurenhammer, F. 1987c. Recognising polytopical cell complexes and constructing projection polyhedra. Journal of Symbolic Computation 3, 3, 249-255.

Babaioff, M., Blumrosen, L., Lambert, N. S., And Reingold, O. 2011. Only valuable experts can be valued. In Proceedings of the 12th ACM conference on Electronic commerce. 221-222.

Babaioff, M., Sharma, Y., And Slivkins, A. 2009. Characterizing truthful multi-armed bandit mechanisms. In Proceedings of the 10th ACM conference on Electronic commerce. 79-88.

Baldwin, E. And Klemperer, P. 2012. Tropical geometry to analyse demand. Tech. rep., Working paper, Oxford University.

Banerjee, A., Guo, X., And Wang, H. 2005. On the optimality of conditional expectation as a Bregman predictor. IEEE Transactions on Information Theory 51, 7, 2664-2669.

Berger, A., Müller, R., And Hossein, N. S. 2010. Path-Monotonicity and Incentive Compatibility. Tech. rep., Maastricht: METEOR, Maastricht Research School of Economics of Technology and Organization.

Berger, A., Müller, R., AND Naeemi, S. 2009. Characterizing incentive compatibility for convex valuations. Algorithmic Game Theory, 24-35.

Bikhchandani, S., Chatteru, S., Lavi, R., Mu'alem, A., Nisan, N., And Sen, A. 2006. Weak Monotonicity Characterizes Deterministic Dominant-Strategy Implementation. Econometrica 74, 4, 1109-1132.

Boissonnat, J.-D., Nielsen, F., And Nock, R. 2007. Bregman Voronoi Diagrams: Properties, Algorithms and Applications. CoRR abs/0709.2196.

Borgwardt, S. And Frongillo, R. M. 2019. Power diagram detection with applications to information elicitation. Journal of Optimization Theory and Applications 181, 1, 184-196. 
Borwein, J. M. And VanderwerfF, J. D. 2010. Convex Functions: Constructions, Characterizations and Counterexamples. Cambridge University Press.

Boutilier, C. 2012. Eliciting Forecasts from Self-interested Experts: Scoring Rules for Decision Makers.

Cai, Y., Mahdian, M., Mehta, A., and Waggoner, B. 2013. Designing Markets for Daily Deals. Preprint.

CARbajal, J. C. AND Ely, J. C. 2012. Mechanism design without revenue equivalence. Journal of Economic Theory.

Carroll, G. 2012. When Are Local Incentive Constraints Sufficient? Econometrica 80, 2, 661-686.

Chambers, C. P. And Lambert, N. S. 2014. Dynamically eliciting unobservable information. In Proceedings of the fifteenth ACM conference on Economics and computation. ACM, 987-988.

Chen, Y., Kash, I., Ruberry, M., and Shnayder, V. 2011. Decision Markets With Good Incentives. Internet and Network Economics, 72-83.

Chen, Y. And Kash, I. A. 2011. Information Elicitation for Decision Making. AAMAS.

Chen, Y., Ruberry, M., and Wortman Vaughan, J. 2013. Cost function market makers for measurable spaces. In Proceedings of the fourteenth ACM conference on Electronic commerce. 785-802.

Chen, Y. And Vaughan, J. 2010. A new understanding of prediction markets via no-regret learning. In Proceedings of the 11th ACM conference on Electronic commerce. 189-198.

Cid-Sueiro, J. 2012. Proper losses for learning from partial labels. In Advances in Neural Information Processing Systems 25. 1574-1582.

Diakonikolas, I., Papadimitriou, C., Pierrakos, G., And Singer, Y. 2012. Efficiency-revenue trade-offs in auctions. In Automata, Languages, and Programming. Springer, 488-499.

Fang, F., Stinchcombe, M. B., And Whinston, A. B. 2010. Proper scoring rules with arbitrary value functions. Journal of Mathematical Economics 46, 6, 1200-1210.

Feige, U. And Tennenholtz, M. 2010. Responsive Lotteries. Algorithmic Game Theory 6386, 150-161.

Feldman, J. And Muthukrishnan, S. 2008. Algorithmic Methods for Sponsored Search Advertising. In Performance Modeling and Engineering, Z. Liu and C. H. Xia, Eds. Springer US, 91-122.

Fiat, A., Karlin, A., Koutsoupias, E., And Vidali, A. 2013. Approaching Utopia: strong truthfulness and externality-resistant mechanisms. In Proceedings of the 4 th conference on Innovations in Theoretical Computer Science. 221-230.

Frongillo, R. And Kash, I. 2015a. Vector-Valued Property Elicitation. In Proceedings of the 28th Conference on Learning Theory. 1-18.

Frongillo, R. And Kash, I. A. 2015b. On Elicitation Complexity. In Advances in Neural Information Processing Systems 29.

Frongillo, R. And Witkowski, J. 2016. A Geometric Method to Construct Minimal Peer Prediction Mechanisms. In Thirtieth AAAI Conference on Artificial Intelligence (AAAI).

Gneiting, T. 2011. Making and Evaluating Point Forecasts. Journal of the American Statistical Association 106, 494, 746-762.

Gneiting, T. And Katzfuss, M. 2014. Probabilistic forecasting. Annual Review of Statistics and Its Application 1, 125-151.

Gneiting, T. And Raftery, A. E. 2007. Strictly proper scoring rules, prediction, and estimation. Journal of the American Statistical Association 102, 477, 359-378.

Grant, K. And Gneiting, T. 2013. Consistent scoring functions for quantiles. In From Probability to Statistics and Back: High-Dimensional Models and Processes-A Festschrift in Honor of Jon A. Wellner. Institute of Mathematical Statistics, 163-173.

Halpern, J. 2003. Reasoning about uncertainty. MIT Press.

HANson, R. 2003. Combinatorial Information Market Design. Information Systems Frontiers 5, 1, 107-119.

Heydenreich, B., Müller, R., Uetz, M., And Vohra, R. V. 2009. Characterization of revenue equivalence. Econometrica 77, 1, 307-316.

Ioffe, A. D. And Tikhomirov, V. M. 1979. Theory of extremal problems. North-Holland Pub. Co. ; sole distributors for the U.S.A. and Canada, Elsevier North-Holland, Amsterdam; New York; New York.

IyeR, R. AND Bilmes, J. 2013. The Lovász-Bregman Divergence and connections to rank aggregation, clustering, and web ranking: Extended Version. Uncertainity in Artificial Intelligence.

Jehiel, P. And Moldovanu, B. 2001. Efficient Design with Interdependent Valuations. Econometrica 69, 5, $1237-1259$.

Jehiel, P., Moldovanu, B., And Stacchetti, E. 1996. How (Not) to Sell Nuclear Weapons. The American Economic Review 86, 4, pp. 814-829. 
Jehiel, P., Moldovanu, B., And Stacchetti, E. 1999. Multidimensional Mechanism Design for Auctions with Externalities. Journal of Economic Theory 85, 2, 258 - 293.

Kos, N. And Messner, M. 2012. Extremal incentive compatible transfers. Journal of Economic Theory.

Krishna, V. And Maenner, E. 2001. Convex Potentials with an Application to Mechanism Design. Econometrica 69, 4, 1113-1119.

LaI, H.-C. And Lin, L.-J. 1988. The Fenchel-Moreau theorem for set functions. Proceedings of the American Mathematical Society, 85-90.

Lambert, N. 2018. Elicitation and Evaluation of Statistical Forecasts. Preprint.

Lambert, N. S., Pennock, D. M., And Shoham, Y. 2008. Eliciting properties of probability distributions. In Proceedings of the 9th ACM Conference on Electronic Commerce. 129-138.

Lambert, N. S. and Shoham, Y. 2009. Eliciting truthful answers to multiple-choice questions. In Proceedings of the 10th ACM conference on Electronic commerce. 109-118.

McAfee, R. And McMillan, J. 1988. Multidimensional incentive compatibility and mechanism design. Journal of Economic Theory 46, 2, 335-354.

Milgrom, P. And Segal, I. 2002. Envelope Theorems for Arbitrary Choice Sets. Econometrica 70, 2, 583-601.

Myerson, R. B. 1981. Optimal auction design. Mathematics of operations research, 58-73.

Müller, R., Perea, A., And Wolf, S. 2007. Weak monotonicity and Bayes-Nash incentive compatibility. Games and Economic Behavior 61, 2, 344-358.

Negahban, S. N., Ravikumar, P., Wainwright, M. J., and Yu, B. 2010. A unified framework for highdimensional analysis of M-estimators with decomposable regularizers. arXiv preprint arXiv:1010.2731.

Nielsen, F. And Garcia, V. 2009. Statistical exponential families: A digest with flash cards. arXiv:0911.4863 [cs]. arXiv: 0911.4863.

OK, E. A. 2007. Real analysis with economic applications. Vol. 10. Princeton University Press.

Osband, K. And Reichelstein, S. 1985. Information-eliciting compensation schemes. Journal of Public Economics 27, 1, 107-115.

Osband, K. H. 1985. Providing Incentives for Better Cost Forecasting. University of California, Berkeley.

Othman, A. And Sandholm, T. 2010. Decision rules and decision markets. In Proceedings of the 9th International Conference on Autonomous Agents and Multiagent Systems. Vol. 1. 625-632.

Peters, H. J. M. And Wakker, P. P. 1987. Convex Functions on Non-Convex Domains. Economics Letters 22, 2-3, 251-255.

Rochet, J.-C. 1985. The taxation principle and multi-time hamilton-jacobi equations. Journal of Mathematical Economics 14, 2, 113-128.

Rochet, J. C. 1987. A necessary and sufficient condition for rationalizability in a quasi-linear context. Journal of Mathematical Economics 16, 2, 191-200.

Rockafellar, R. 1997. Convex analysis. Princeton Mathematics Series Series, vol. 28. Princeton University Press.

SAKS, M. AND YU, L. 2005. Weak monotonicity suffices for truthfulness on convex domains. In Proceedings of the 6th ACM conference on Electronic commerce. 286-293.

SAVAGE, L. 1971. Elicitation of personal probabilities and expectations. Journal of the American Statistical Association, 783-801.

Shah, N. B. And Zhou, D. 2015. Double or nothing: Multiplicative incentive mechanisms for crowdsourcing. In Advances in Neural Information Processing Systems. 1-9.

Steinwart, I., Pasin, C., Williamson, R., and Zhang, S. 2014. Elicitation and Identification of Properties. In Proceedings of The 27th Conference on Learning Theory. 482-526.

Urruty, J.-B. H. And Lemaréchal, C. 2001. Fundamentals of Convex Analysis. Springer.

Van Manen, M. And Siersma, D. 2005. Power diagrams and their applications. arXiv preprint math/0508037.

Vohra, R. V. 2011. Mechanism design: a linear programming approach. Cambridge University Press, Cambridge; New York.

Witkowski, J., Bachrach, Y., Key, P., And Parkes, D. C. 2013. Dwelling on the negative: Incentivizing effort in peer prediction. In First AAAI Conference on Human Computation and Crowdsourcing.

YAN, M. 2012. Extension of convex functions. arXiv preprint arXiv:1207.0944. 